\title{
Mathematical Explanations of Physical Phenomena
}

\author{
Manuel A. Barrantes \\ Lima, Perú
}

\begin{abstract}
B.A. Philosophy, Pontifical Catholic University of Perú
M.A. Philosophy, University of Virginia

A Dissertation Presented to the Graduate

Faculty of the University of Virginia

in Candidacy for the Degree of Doctor of Philosophy
\end{abstract}

\author{
Department of Philosophy \\ University of Virginia
}

October 2017 
To Bernardo Haour, the philosopher 


\section{Acknowledgments}

I would like to thank my advisor, professor Paul Humphreys, for the time and patience he has put in discussing this project with me. In the spring of 2011, professor Humphreys offered me to have a tutorial instruction where we discussed issues pertaining to the applicability of mathematics in science. That opportunity came at the right time for me, and it was a turning point in my career. I am truly thankful for it, and for all that came next.

I would also like to thank the other members of my committee: professors James Cargile, Otávio Bueno, Karen Parshall, and Ross Cameron. Professor Cargile has been a constant source of encouragement, and our conversations have been crucial for my understanding of the scope of my own project. Professor Bueno's Inferential Conception is a central part of my dissertation, and he has been very generous in discussing it with me on several occasions. In addition, my thanks go to professors Parshall and Cameron, for reading my work and for their comments.

Special thanks are due to professors Jorge Secada, Bernardo Haour, and to Juan Durán. Professor Secada encouraged me to come to the US as a philosophy graduate student and helped me navigate this process as a foreign student. Juan Durán has been an incredible interlocutor during never-ending hours of philosophical discussions at the most unlikely places. Professor Haour showed me with his example that it is possible to be a rigorous philosopher who is passionately involved in social change, and that has been an immense source of motivation.

I would also like to acknowledge the financial support of the Department of Spanish, Italian and Portuguese at the University of Virginia, and the Department of Philosophy and Religion at James Madison University. Without this support, it would have been impossible for me either to start or finish this project.

Finally, thanks to my family, especially to El Castor and Lil, for inspiring me with their work ethic, optimism, and sense of humor. And of course, my thanks to my wife, the strongest person I know, go in Spanish: La hicimos Fex. Tú y yo. Alucinante. Gracias. 


\section{TABLE OF CONTENTS}

$\S 1$ Introduction

$\S 2$. Outline

CHAPTER 1. Scientific Explanation: Modal Information and the Right Level of Description

$\S 3$. Introduction

$\S 4$. Hempel's Deductive-Nomological Model

$\S 5$. Problems with the DN Model

$\S 6$. Causation vs. Logical Derivation

$\S 7$. Salmon's Causal-Mechanical Model

$\S 8$. Problems with the CM Model

$\S 9$. Woodward's Interventionist Account

$\S 10$. Conclusion

\section{CHAPTER 2. Mathematical Models in Science}

$\S 11$. Introduction

$\S 12$. Abstraction and Idealization in Mathematical Modeling

$\S 13$. Pincock's Mapping Account of the Applicability of Mathematics

$\S 14$. The Inferential Conception of the Applicability of Mathematics

$\S 15$. Mathematics and Scientific Explanation

$\S 16$. Conclusion

\section{CHAPTER 3. An Account of Mathematical Explanations of Physical Phenomena}

$\S 17$. Introduction

$\S 18$. Case study 1. The Bridges of Königsberg

$\S 19$. Case study 2. The Cicadas

$\S 20$. An account of MEPP

$\S 21$. Cases revisited

$\S 22$. MEPPs as noncausal explanations

$\S 23$. Conclusion 


\section{CHAPTER 4: Other Accounts of MEPPs}

$\S 24$. Introduction

$\S 25$. Descriptive vs Representational views of MEPPs

$\S 26$. Mark Steiner: MEPPs and explanatory proofs

$\S 27$. Alan Baker: MEPPs and indispensability

$\S 28$. Aidan Lyon: MEPPs and program explanations

$\S 29$. Marc Lange: MEPPs and mathematical necessities

$\S 30$. Christopher Pincock: MEPPs and mathematical structures

$\S 31$. Conclusion

\section{CHAPTER 5. The Indispensability Argument}

$\S 32$. Introduction

$\S 33$. Quine's indispensability argument

$\S 34$. Problems with confirmational holism

$\S 35$. The explanatory Indispensability Argument

$\S 36$. The Enhanced Indispensability Argument

$\S 37$. Are MEPPs the best explanations?

$\S 38$. The EIA and practical indispensability

$\S 39$. Conclusion

\section{REFERENCES}




\section{$\S 1$. Introduction}

The most natural way of understanding the role of mathematics in scientific explanations is as representing physical explanatory facts and helping to draw inferences about those facts. According to Joseph Melia, for example, because mathematics offers good representations of the physical world, some scientific explanations require the use of mathematics, but this does not mean that mathematics is in itself explanatory. If we say, for example: ' $F$ occurs because $P$ is $\sqrt{2}$ meters long', despite the fact that we are mentioning the number $\sqrt{2}$ in the explanation, it is the actual physical length of object $P$, not the real number $\sqrt{2}$ by which we represent it, that does the real explanatory work (cf. Melia 2002, 76). The main idea is that mathematical statements feature in scientific explanations because of these representational and inferential roles. By performing derivations over these mathematical statements, we can learn how the relevant physical facts explain the explanandum.

But it has recently been argued that mathematics may be able to do more than this, that there can be mathematical explanations of physical phenomena (MEPP). In recent years, there has been much discussion about the nature of these MEPPs. Authors wonder what exactly it means for mathematics to explain a physical phenomenon; whether MEPPs are genuinely different from ordinary scientific explanations that use mathematics; and, if MEPPs are indeed different, which ontological consequences follow from the fact that there are MEPPs in science.

Many purported cases have been advanced in recent literature, and there have been many attempts to determine what exactly the distinctive feature of each of these cases is, and whether they all belong to the same category; and although there is no current consensus, most authors agree that in these explanations mathematics is involved in a special way. For example, almost all accounts agree that these are scientific explanations that depend on the mathematical model they use in the explanans in a way such that without the mathematical model these explanations would not stand. In other words, the mathematical part of these explanations is indispensable for the explanation to work as an explanation.

However, many authors have gone further, and claim that in these cases mathematics itself is playing an explanatory role in science. In fact, some even suggest that MEPPs ontologically commit us to the existence of mathematical entities, following a new version of the Indispensability Argument. If mathematical statements feature indispensably in some scientific explanations, then, if we are scientific realists, we ought to be committed to the existence of the mathematical entities that make those mathematical statements true (just as, say, explanations of quantum phenomena commit us to the existence of subatomic particles).

I think, however, that we should not go that far. In my dissertation, I offer an account of MEPPs that emphasizes the representational role of mathematics. That is, I argue that in MEPPs the role of mathematics is (merely) to represent physical facts, but I also maintain that these explanations are special. I combine elements of James Woodward's counterfactual account of scientific explanation, and Otávio Bueno, Marc Colyvan, and Stephen French's Inferential Conception of the Applicability of Mathematics. The main aspects of my account are the notions 
of optimal representation and explanatory mathematical derivations. My goal is to advance an account of MEPPs that is counterfactual, noncausal, and where the role of mathematics is representational.

The dissertation is divided in five chapters. I give some background to the topic of scientific explanation and introduce Woodward's account in chapter 1 . In chapter 2 I discuss the role of mathematics in scientific explanation. In chapter 3 I introduce my own account of MEPPs. In chapter 4 I discuss other accounts of MEPPs; and in chapter 5 I show that the existence of MEPPS does not justify mathematical realism.

\section{$\S 2$. Outline}

Chapter 1. This chapter is an overview of the philosophical debate on scientific explanation, and introduces the notions that will be important throughout the dissertation, namely, explanation, causation, and invariance, and more specifically, the notions of explanatory asymmetry, explanatory relevance, and modality.

I first present Carl Hempel's Deductive-Nomological model, and discuss two problems of this view. Hempel's model allows irrelevant facts as explanatory, and it cannot account for the asymmetry between the explanandum and the explanans of a scientific explanation. Next, I discuss the main motivations for adopting causal accounts of scientific explanation. I introduce Wesley Salmon's notion of causation and his Causal-Mechanical model of scientific explanation, and discuss some problems of this view, the most important being that, because of its restrictive definition of causation, the CM model does not fully solve the problem of relevance. I next address James Woodward's counterfactual account of causation and scientific explanation. I argue that this account is better equipped to deal with the problem of relevance. Crucial for understanding this view is the notion of the right level of description, which is that for identifying the aspects relevant to the occurrence of the phenomenon to be explained, sometimes we must refrain from describing all the details of the overall situation. In those cases, what we gain is a better understanding of the explanandum, by highlighting its modal strength. As I will show in further chapters, this feature of successful scientific explanations is fundamental for fully grasping the role of mathematics in MEPPs. And yet, Woodward's model, as it stands, is not capable of accounting for these MEPPs.

Chapter 2. This chapter focuses on the role of mathematical models in scientific representation and scientific explanation. The discussion of this chapter provides the framework for my account of Mathematical Explanations of Physical Phenomena, which is the focus of chapters 3 and 4.

I begin this chapter presenting the notion of mathematical model, including a conceptual distinction between abstraction, approximation, and idealization. I also discuss the different kinds of models there are, based on the representational ideals of the scientists who use them. I next analyze two accounts of the application of mathematics: Christopher Pincock's mapping account, which highlights the importance of the structural resemblance between the model and the target system; and Bueno, Colyvan, and French's Inferential Conception, which, in addition, emphasizes the context of application, as well as the role of the interpretation the users of the model assign 
to its mathematical structures. The main point here is that, without a proper interpretation, mathematics says little about the physical world. Next, I use the Inferential Conception to clarify the role of mathematics in scientific explanation, and discuss a criticism by Robert Batterman (2010). I finish the chapter by presenting a possible shortcoming of the Inferential Conception, which is that this view does not distinguish between explanatory and non-explanatory mathematical derivations.

Chapter 3. In this chapter, I introduce my account of Mathematical Explanations of Physical Phenomena (MEPPs). On my view, MEPPs are counterfactual explanations. And despite the fact that MEPPs are explanations of empirical phenomena, they are noncausal.

I start by presenting and analyzing two cases that have been much debated in the literature: the impossibility of performing a trip over all the bridges of Königsberg without retracing one's steps, whose explanation appeals to a property of non-Eulerian graphs (Pincock 2007); and the prime numbered life-cycle of some species of periodical cicadas, whose explanation appeals to a property of prime numbers (Baker 2005). I next introduce my account of MEPPs in terms of the notions of optimal representation (as opposed to the improvable representations that operate in most applications of mathematics); deformations (as opposed to the causal interventions of Woodward's account); and explanatory mathematical derivations (as opposed to Mark Steiner's notion of explanatory proof), and revisit my cases to illustrate my view. I finish the chapter discussing the sense in which MEPPs are different from ordinary scientific explanations, and how MEPPs account for the explanatory directionality.

Chapter 4. In this chapter, I distinguish between descriptive and representational accounts of MEPPs. The former presuppose that the physical world has mathematical features which are then described by the mathematical part of MEPPs; the latter do not need that assumption. In this chapter I defend my representational account against alternative views of MEPPs.

I first introduce the distinction between descriptive and representational views of MEPPs. Next, I discuss Steiner's view of MEPPs, which is known as the transmission view. Steiner says that at the core of every MEPP there is an explanatory proof of a mathematical theorem. Within the philosophy of mathematics literature, however, the notion of explanatory proof is controversial, and I take it as an advantage of my own view that it does not require explanatory mathematical proofs. In addition, Steiner does not specify which kind of scientific questions may be amenable to be answered by a MEPP. My view does, and that is another advantage over Steiner's. I then argue, against Alan Baker, that although MEPPs do not require explanatory proofs, they do require some explanatory information coming from their mathematical component. Next, I focus on the modal accounts of MEPPs. Aidan Lyon argues that MEPPs appeal to higher order mathematical features of the explanandum, and Marc Lange argues that MEPPs point to relations of mathematical necessity in the physical world. I reject these views by pointing out first that they do not specify the sense in which those mathematical features may be explanatory, and secondly, that they do not convincingly establish the need of positing the existence of relations of mathematical necessity in the physical world in order to make sense of MEPPs. Finally, I analyze Christopher Pincock's view of MEPPs as abstract explanations, according to which MEPPs work by 
virtue of the mathematical structures underlying the physical world. I show first that the view that mathematical structures may have physical instantiations is unfounded, and secondly that even if this was correct, this view fails to explicate the sense in which MEPPs provide explanatory information.

Chapter 5. The Indispensability Argument (IA) relies on the applicability of mathematics in science to support mathematical realism. The explanatory version of the IA focuses on the indispensability of mathematics in scientific explanations, and relies on the principle of Inference to the Best Explanation (IBE) to justify its conclusion. The idea of this explanatory IA is that if we believe in the existence of the concrete unobservable posits that feature in our best scientific explanations, we should also believe in the mathematical posits of those explanations. However, against the explanatory IA it has been objected that the role of mathematical posits in scientific explanations is to represent concrete explanatory facts and help draw inferences about those facts, but that mathematical posits, by themselves, are not explanatory. This has been taken as a weakness of the explanatory IA, and so a new version of the argument has been recently advanced, known as the 'Enhanced Indispensability Argument' (EIA). The EIA relies on the fact that there are mathematical explanations of physical phenomena (MEPPs) to support the claim that mathematics can play an explanatory role beyond its representational and inferential roles. According to the EIA, if we apply the IBE principle to MEPPs, mathematical realism would be justified. In this chapter I use my account of MEPPs to refute the EIA.

I start by introducing the original IA, and discuss criticisms by Charles Parsons, Elliott Sober and Penelope Maddy. Next, I present the explanatory IA, and, after presenting criticisms from Hartry Field and Joseph Melia, I discuss the motivation for the Enhanced Indispensability Argument (EIA). I then use the account of MEPPs that I have developed in the previous two chapters to show that the EIA fails. First, I show that for mathematics being indispensable in explanations in a way that carries ontological commitments, the explanandum must already be committed to the existence of mathematical properties and entities, which renders the EIA circular. Next, I show that the indispensability of the mathematical component in MEPPs is pragmatic, and that, ultimately the role of mathematics is representational and inferential. For this reason, the existence of MEPPs does not have the ontological consequences required by the EIA. My goal is not to directly defend mathematical nominalism, but to show that the existence of MEPPs does not support mathematical realism. 


\section{CHAPTER 1. Scientific Explanation: Modal Information and the Right Level of Description}

\section{§3. Introduction}

The main goal of this dissertation is to advance an account of Mathematical Explanations of Physical Phenomena (MEPPs) that is counterfactual, and noncausal. This introductory chapter is an overview of the philosophical debate on scientific explanation, and introduces the notions that will be important throughout the dissertation, namely, explanation, causation, and invariance, and more specifically, the notions of explanatory asymmetry, explanatory relevance, and modality.

I first present Carl Hempel's Deductive-Nomological model ( $\$ 4)$, and discuss two problems of this view (§5). Hempel’s model allows irrelevant facts as explanatory, and it cannot account for the asymmetry between the explanandum and the explanans of a scientific explanation. Next, I discuss the main motivations for adopting causal accounts of scientific explanation (§6). I introduce Wesley Salmon's notion of causation and his Causal-Mechanical model of scientific explanation (§7), and discuss some problems of this view (§8), the most important being that, because of its restrictive definition of causation, the CM model does not fully solve the problem of relevance. I next address James Woodward's counterfactual account of causation and scientific explanation (§9). I argue that this account is better equipped to deal with the problem of relevance. Crucial for understanding this view is the notion of the right level of description, which is that for identifying the aspects relevant to the occurrence of the phenomenon to be explained, sometimes we must refrain from describing all the details of the overall situation. In those cases, what we gain is a better understanding of the explanandum, by highlighting its modal strength. As I will show in further chapters, this feature of successful scientific explanations is fundamental for fully grasping the role of mathematics in MEPPs. And yet, Woodward's model, as it stands, is not capable of accounting for these MEPPs.

\section{$\S 4$. Hempel’s Deductive-Nomological Model}

According to Wesley Salmon, there are three different kinds of accounts of scientific explanation: epistemic, modal, and ontic:

Epistemic accounts: An explanation is an argument to the effect that the explanandumstatement must be nominally expectable given the statements in the explanans.

Modal accounts: An explanation must show that a given event had to occur, given the facts cited in the explanans.

Ontic accounts: An explanation consists in showing how the explanandum fits into the network of objective patterns of dependence in the world (Salmon 1984, 15-18) ${ }^{1}$

\footnotetext{
${ }^{1}$ For the most part, I will ignore pragmatic accounts of explanation.
} 
In this section I will discuss Hempel's epistemic account. In the following sections I will discuss two ontic accounts (those of Salmon and Woodward). Mark Lange's modal account will be addressed in chapter 4 .

At the beginning of the $20^{\text {th }}$ century, most positivist philosophers agreed that science should not aim at providing explanations. Explanation was thought to require an understanding of the deep nature of things, and this was thought to be outside the bounds of science. The scientific enterprise was to describe what we observe of the physical world, and in that sense description was thought to be the opposite of explanation. Karl Pearson, for example, said that "[n]obody now believes that science explains anything; we all look upon it as a shorthand description, as an economy of thought" ([1911] 1957, xi, emphasis in the original; quoted in Salmon 2000, 313).

In 1948, however, Carl Hempel and Paul Oppenheim published an influential essay providing an account of scientific explanation that changed the views of philosophers in this matter (Hempel \& Oppenheim 1948). After further elaboration by Hempel (e.g. Hempel 1965), this view became the most important philosophical account of scientific explanation until the 70's, to the point of being considered 'the received view' of scientific explanation. Hempel's DeductiveNomological (DN) model is an attempt to explicate the notion of scientific explanation while avoiding (to the eyes of positivist philosophers) metaphysically dubious concepts such as causation and production. Scientific explanations, according to this model, are deductive arguments that are given in order to answer a why-question.

The latter statement needs to be qualified. Some why-questions are explanation-seeking, and some are evidence-seeking. If we interpret 'why p?' as an evidence-seeking question, we should look for reasons to believe p. But if we interpret 'why p?' as an explanation-seeking question, we must presuppose that $p$ is true, and we should focus on the events that lead to the occurrence of whatever is described by $p$. Now, in many cases a why-question can be interpreted either way, so whether a why-question is evidence-seeking or explanation-seeking is not straightforward. Consider the following example due to Salmon ([1989] 2006, 7):

\section{p: Distant galaxies receding from us}

If we take why $p$ as an evidence-seeking why-question, we may say that this is because the light coming from these galaxies shifted to the red end of the spectrum. However, if we take why $p$ as an explanation-seeking why-question we may answer, for example, that this is a consequence of the Big Bang, and we may proceed to show how this happened. The crucial difference is that in the former case we want reasons to believe in $p$, whereas in the latter case, we take $p$ to be true; we are not looking for reasons to believe it. Although science provides answers to both kinds of questions, the philosophical debate on scientific explanation is focused on why-questions interpreted as explanation-seeking. It is a basic requirement for any account of scientific 
explanation that the explanandum (the proposition describing the fact to be explained) must be true (cf. Salmon [1989] 2006, 7) ${ }^{2}$.

According to the DN model, a scientific explanation is a valid deductive argument that answers an explanatory why-question. The premises of the argument are known as the explanans (the set of propositions describing the facts that do the explanatory work), and the conclusion of the argument is the explanandum. The structure underlying successful scientific explanations is that of a valid deductive argument the conclusion of which is the explanandum. Now, it is important to note that Hempel's view is not that all explanations in science are given in DN form, but that a DN structure (or its variations; see footnote 4) underlies any given scientific explanation.

There are general and empirical conditions for a DN explanation. The general conditions are that:

i. The explanation must be a valid deductive argument

ii. The explanans must contain at least one general law that is essential for the validity of the argument

iii. The explanans must have empirical content (and so the general law mentioned in (ii) must be a law of nature) ${ }^{3}$

The empirical condition is that:

iv. The sentences constituting the explanans must be true

The explanation consists in subsuming the explanandum under the laws cited in the explanans. The idea is, in Hempel words, that scientific explanations "provide a systematic understanding of empirical phenomena by showing that they fit into a nomic nexus" $(1965,488)$. Thus, the general form of a DN explanation is the following:

$$
\begin{array}{lll}
\text { Explanans: } & \mathrm{C}_{1}, \mathrm{C}_{2} \ldots \mathrm{C}_{\mathrm{k}} \quad \text { [particular circumstances] } \\
& \mathrm{L}_{1}, \mathrm{~L}_{2} \ldots \mathrm{L}_{r} \quad \text { [general laws] } \\
\text { Explanandum: } & \mathrm{E} & \text { [description of the phenomenon to be explained] }
\end{array}
$$

\footnotetext{
${ }^{2}$ This does not mean, as we will see below in section $\S 35$, that all the entities over which this proposition quantifies exist. It is the physical fact described by the explanandum statement that must be 'real', i.e. a fact.

${ }^{3}$ Alan Baker (2005) says that MEPPs accommodate the DN Model, because, for him, MEPPs require "purely mathematical laws" $(2005,235)$, but I believe condition (iii) rules out this possibility. Purely mathematical laws (if they can be so called) lack empirical content. More on this in section $§ 27$.
} 
Wesley Salmon calls accounts like Hempel's epistemic, due to their emphasis in the nomic expectability of the explanandum given the explanatory facts mentioned in the explanans. Salmon emphasizes that knowledge of these facts renders the explanandum expectable, because there is a relation of logical necessity between the explanans statements and the explanandum statement $(1984,16)$. In Hempel's words, the purpose of a scientific explanation is to show that "given the particular circumstances and the laws in question, the occurrence of the phenomenon was to be expected" (Hempel 1965, 337). This condition of nomic expectability is the reason why, in this model, explanations have the same logical form (and are on a par with) predictions. If it had been given before the occurrence of the fact described by the explanandum, a good explanation would have been a good prediction; conversely, a good prediction is a potential explanation. This has been called 'the symmetry thesis', and it is a crucial element in the DN model ${ }^{4}$. Consider the following case:

BOUNCING BALL CASE: Why did a ping pong ball bounce around $26,8 \mathrm{~cm}$ when it was dropped from $30,5 \mathrm{~cm}$ height?

The first thing to note is that this is an explanation-seeking why-question. We are assuming that it is true that the ball bounced around $26,8 \mathrm{~cm}$. Ignoring aerodynamical effects, a common textbook explanation would derive the speed right before the collision with the ground (V) from the kinematic laws of motion; the speed right after the collision $\left(V^{\prime}\right)$ from the laws of conservation of momentum and semi elastic collisions; and the maximum height after the bouncing $\left(h_{f}\right)$ from $V$ and the kinematic laws. The DN structure of the textbook explanation would be the following:

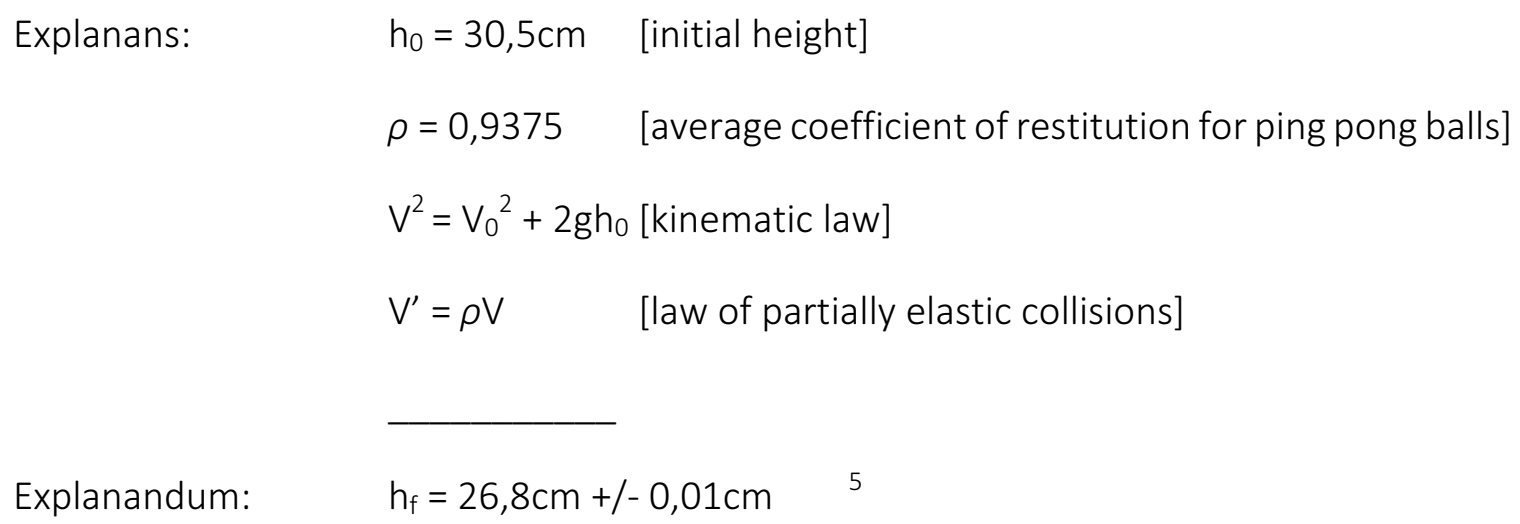

\footnotetext{
${ }^{4}$ Hempel's model is not restricted to explanations of particular events, nor it is confined to deterministic contexts. It can explicate explanations of general regularities both using deterministic (Deductive Nomological (DN) model) and statistical laws (Deductive Statistical (DS) model), as well as particular events both using deterministic (Deductive Nomological (DN) model) and statistical laws (Inductive Statistical (IS) model), the latter by showing that the explanandum has high inductive probability given the explanatory facts.

${ }^{5}$ With $h_{0}=30,5 \mathrm{~cm} ; V_{0}=0$ and $V^{2}=V_{0}^{2}+2 g h_{0}$ the speed before collision is $V=\sqrt{0,305 \times 9,8 \cdot 2}$. This result, along with $\rho=0.9375$ and $V^{\prime}=\rho V$, gives us a speed after collision of $V^{\prime}=0,9375 \times \sqrt{0,305 \times 9,8 \cdot 2}$. In turn, this result, along with $V_{f}^{2}=V^{\prime 2}+2 g h_{f}$, gives us a final height $h_{f}=0,26806 \mathrm{~m}=26,806 \mathrm{~cm}$.
} 
This explanation of course can be improved with information about the aerodynamics of the ball and the air conditions at the time of the dropping. In fact, the cited law of partially elastic collisions is an instance of a more general law of conservation of momentum and kinetic energy. The empirically determined coefficient of restitution $\rho$ could in principle be substituted with information about the material constitution of the ball and the rigidity of the ground. But still, this case exemplifies the general form of DN explanations. It includes two laws of nature and the relevant initial conditions, and it shows how the explanandum is an instance of the general cases described by those laws. Moreover, it shows that, given the initial conditions, the explanandum was expected to occur. That is, given the values of $h_{0}$ and $\rho$, we could have predicted that the final height would be $26,8 \mathrm{~cm}$ when dropping the ball from a $30,5 \mathrm{~cm}$ height.

\section{$\S 5$. Problems with the DN Model}

There are many famous counterexamples to the DN model. In this section I will focus on two, which are associated respectively to the asymmetry between the explanans and the explanandum, and the relevance of the explanans for explaining the explanandum. ${ }^{6}$

\subsection{The asymmetry between explanans and explanandum}

One problem with the DN model is that it cannot account for a seemed asymmetry between explanans and the explanandum. The most famous example to show this is the flagpole case:

FLAGPOLE CASE: Why does a flagpole of height h cast a shadow of length L? (cf. Salmon 2006/1989, 47).

A valid DN explanation of this should mention the height of the flagpole, the elevation of the sun, and the laws of trigonometry, given the rectilinear propagation of light. ${ }^{7}$ But suppose that we want to know why the height of the flagpole is $h$, and we deduce it from the elevation of the sun, the laws of trigonometry, and the length of the shadow. This alleged explanation appeals to the length of the shadow to explain the height of the flagpole, which is incorrect. The height of the

\footnotetext{
${ }^{6}$ Another famous problem with the DN model has to do with the requirement of nomic expectability. As we saw, the symmetry between explanation and prediction requires that the fact to be explained was expected to occur (or at least had a high probability of occurring), given the facts cited in the explanans. However, this requirement is not met by those perfectly acceptable probabilistic explanations that do not confer high inductive probability to the explanandum. Consider the case of mother-to-infant HIV transmission. It is estimated that, without receiving retrovirals, around $20 \%$ of HIV-infected pregnant women transmit the disease to their children. If a newborn is infected with HIV, the mother being infected would be an explanatory factor, even though, on the basis of the mother being infected, one could not have predicted the infection of her child. (Another such a case, widely discussed in the literature, is the development of paresis in syphilis patients (e.g. Salmon [1989] 2006, 49; Woodward 2003, 155)). This problem of the DN model is not directly related to my discussion.

${ }^{7}$ Although mathematical laws lack empirical content, citing them in this case does not violate condition (iii) of section §4. Under certain circumstances the trajectory of light can be represented by the laws of trigonometry. In that sense, the cited laws are describing an (approximation of) empirical facts. More of this in sections $\S 15$ and $\S 27$.
} 
flagpole is better explained by the intentions of the people who built it, its position with respect to the ground and the sun, as well as the properties of its own material components. But despite this, it fits the DN definition of scientific explanation. Another such an example is the relationship between symptoms and diseases. One usually takes symptoms as evidence of a disease; and the disease as explaining the symptoms. The DN model allows symptoms to explain diseases because it ignores the asymmetric dependence between the two. This problem generalizes to many other situations. On the DN model, for example, future events can explain past ones! Craver \& Povich (2017) call these alleged explanations 'reverse cases'. The DN model, then, is too permissive, for it cannot rule out reverse cases as bad explanations because it cannot account for the asymmetry between the explanandum and the explanans.

Now, in a widely-known twist of the flagpole case, Bas C. van Fraassen argues that there are cases where the length of the shadow can indeed explain the height. In his famous story 'The Tower and the Shadow', van Fraassen presents a situation of a Chevalier who wanted to cover a terrace under a shadow, so he built a tower that casts a shadow of the required length $(1980,132$ 134). In this case, since the length of the shadow is fixed, it determines the height the Chevalier needs. Therefore, the length of the shadow would explain, in Van Fraassen's sense, why the Tower was built with such-and-such height. Van Fraassen's main point is that there is no explanatory asymmetry simpliciter; rather, explanatory asymmetry is a context-dependent notion, relative to the specific interests of the person doing the explaining. Van Fraassen's is a pragmatic account of scientific explanation.

However, one may argue, as Salmon does, that even in this case there is indeed an explanatory asymmetry that is independent of the explanatory context. Salmon argues that it is not the shadow, but the beliefs and desires of the Chevalier, along with facts about the Tower's material constitution, and the rectilinear propagation of light that explain why the Tower was built with such a height (cf. Salmon [1989] 2006, 144). As we will see below, Salmon's account of scientific explanation is ontic. Ontic accounts exploit objective patterns dependence. ${ }^{8}$

\subsection{The relevance of the explanans}

Another serious problem with the DN model is that it allows irrelevant factors as explanatory. Consider the following case:

BIRTH-CONTROL PILLS CASE: Why did Mr. Jones, a male, fail to get pregnant? (cf. Salmon [1989] 2006, 50)

A valid DN explanation would be that Mr. Jones took birth-control pills, and any male that takes birth-control pills fails to get pregnant. The generalization g: 'any male that takes birthcontrol pills fails to get pregnant' is an exceptionless regularity that may well be considered a genuine law of nature ${ }^{9}$, so this explanation meets the requirements of the DN model. The problem,

\footnotetext{
${ }^{8}$ As we will see in section $\S 18$, my account of MEPPs is also ontic.

${ }^{9}$ Hempel mentions that for a lawlike statement to be a law (and not merely an accidental generalization) (a) it has to be true; (b) it has to 'pass the counterfactual test'; and (c) It has to be explanatory (cf. Salmon
} 
however, is that the fact that Mr. Jones has been taking birth-control pills is irrelevant to explaining why he failed to get pregnant. Another famous counterexample to the DN model, with respect to the problem of relevance, is the hexed salt case: A sample of salt dissolved because both it was placed into water and it was hexed. Evidently, the hexing (whatever that is) is irrelevant for the dissolution of the salt (cf. Salmon [1989] 2006, 50).

These cases highlight a deep structural problem of the DN model. A valid deductive argument continues to be valid when a new proposition is conjunctively added to one of the premises; but this does not seem to be true for scientific explanations. Explanations should discriminate what is and what is not relevant to the occurrence of the explanandum. This notion of explanatory relevance, however, cannot be captured by the DN model.

\section{§6. Causation vs. Logical Derivation}

In Hempel's model, there is a relation of logical necessity between the statements that describe the facts and laws in the explanans, and the statement that describes the facts in the explanandum. However, the logical derivation of the explanandum from the premises that feature in the explanans is not what makes an explanation successful, for this relation of logical necessity does not necessarily represent explanatory relationships. In particular, it does not necessarily capture the relevant features of the world upon which the explanandum depends.

As we saw, Salmon calls ontic those accounts of scientific explanation that emphasize that successful explanations cite objective features of the world (objective patterns of events) (1984, 18). Most ontic accounts are causal; they take that the basic feature of a successful explanation is that it should mention the cause(s) of the explanandum. Thus, for example, in 1975 Wesley Salmon wrote that "the instance in which [an] event can play an explanatory role is one in which it is cause (or part thereof) of the explanandum event" $(1998,111)$. Note here the shift from the explanans and explanandum being sentences to being facts or events. Explanation is no longer a matter of logical necessity but of physical necessity. What is being emphasized now are objective relationships of dependence in the physical world, not the logical relationships between the statements that describe the world.

Until very recently, that scientific explanations are causal explanations was the new consensus in the philosophy of scientific explanation. However, as I indicated in section $\S 1$, and will see in section $§ 18$, one of the purposes of this dissertation is to contribute to recent challenges to this consensus, by providing an account of mathematical explanations of physical phenomena, which, I argue, are ontic but noncausal explanations. I leave this discussion for further chapters. My purpose here and in the remaining sections of this chapter is to discuss two of the most important causal accounts of scientific explanation.

$2006 / 1989,14)$. Generalization $g$ is true (a); the counterfactual 'if $x$ had been a male that took birth-control pills, $x$ would have failed to get pregnant' is also true (b). Whether g meets (c) is more problematic. I think $\mathrm{g}$ can be used in DN explanations, but it may not be explanatory under Salmon's, because it is not about conservative quantities. 
Causal accounts can successfully deal with some of the problems of Hempel's account. The requisites for a successful logical derivation can be met by cases that do not preserve the asymmetry between explanans and explanandum, or cases that include irrelevant information. For these reasons, merely showing how a particular case falls under a general law is not enough to capture these asymmetries or guarantee the needed explanatory relevance; describing causal relationships, on the other hand, is a way of meeting these requirements. With this in mind, we can see the DN model in a new light. Sometimes the derivation relation between the explanans and the explanandum captures causal relationships between them, and sometimes it does not. When it does not, the derivation fails to provide a successful explanation.

Let us consider the asymmetry problem in more detail. In scientific explanation conceived as logical derivation, the particular facts cited in the explanans and the explanandum can be interchanged. As we have seen, this permissiveness represents one of the strongest motivations for abandoning the DN model and adopting a causal model instead. This asymmetry between the explanans and the explanandum can be captured if we consider the causal relationships that are present in the situation to be explained. In the flagpole case, for example, it is the height that, along with other initial conditions and according to the laws of nature, causes the flagpole to block some light rays, thus casting a shadow of a certain length. Thus, we explain the shadow by appealing to the height (its cause). In the same way, diseases cause symptoms, past events cause future ones, etc., and for that reason the former can be called in to explanations of the latter.

This can also be seen in the bouncing ball case. Although the DN model seemed to have correctly described what was explanatory in this case, it actually missed it. It is not because of the derivation relation between the explanans (initial height, coefficient of restitution, and the laws) and the explanandum (the final bouncing height) that the former explains the latter. If that were the case, we could have explained the initial height by appealing to the final height, which is absurd. It is not the derivation itself, but the causal relationship between the initial height and the final height that provides the asymmetry between the explanandum and the explanans.

As we saw, another problem with Hempel's model was that of relevance. This may not be a problem for causal models (at least prima facie). An event is relevant to explaining another event if the former is causally relevant to the occurrence of the latter. Some biological factors are causally relevant for Mr. Jones' non-pregnancy, but taking birth-control pills is not. Therefore, the formers can explain his non-pregnancy but the latter cannot.

The bouncing ball case also shows this. The DN explanation of the final height appealed to the initial height, some features of the ball and the ground, and the laws that describe how those factors interact. However, if we add to the explanans, say, the fact that the ball is blue, the derivation would still be DN successful, but being blue is actually irrelevant to the bouncing. The idea is that derivations that describe causal relationships can capture the relevance of the explanans. But derivations that do not may mistakenly count irrelevant factors as explanatory.

Now, the concept of causation is indeed philosophically problematic. Bertrand Russell famously said that: 
"The law of causality... like much that passes muster among philosophers, is a relic of a bygone age, surviving, like the monarchy, only because it is erroneously supposed to do no harm" (Russell [1913] 1957, 174; cited in Dowe 2009, 214-15).

Despite this, as we have seen, in the context of scientific explanation the notion of causation is very appealing. In fact, Russell himself did not abandon this notion, but rather redefined it in terms of 'causal lines'. This has been the general rule for causal accounts of scientific explanation: they are always associated with specific definitions of what it means for something to cause something else (cf. e.g. Salmon 1984; Humphreys 1989b; Woodward 2003).

\section{§7. Salmon's Causal-Mechanical Model}

Wesley Salmon's causal mechanical (CM) model states that a scientific explanation must identify the causal relations between the explanandum and the events that caused it. The correspondent account of causation is cashed out in terms of causal processes and causal interactions, which are the basic causal mechanisms by which causal influence is transmitted. Salmon first defined causal processes and interactions using the 'mark transmission' criterion, but later he abandoned it and adopted the 'conserved quantity' criterion.

\subsection{Causation}

\section{a) Mark transmission criterion}

In his (1984), Salmon defined a causal process as anything that is capable of transmitting a mark, and a causal interaction as the mutual modification of two intersecting processes. A mark is a modification of a characteristic $Q$ of a process $P$ by means of a single local interaction at point $A$. The mark is transmitted to point $B$ if $P$ manifests the modification $Q^{\prime}$ at $B$ and at all stages of the process between A and B (cf. Salmon 1984, 148). This principle of mark transmission is counterfactual: the process $P$ would have continued having characteristic $Q$ if the specific marking interaction had not occurred (cf. Salmon 1984, 148). Salmon used this mark transmission criterion to distinguish between causal processes and pseudoprocesses, which are processes incapable of transmitting a mark. Examples of these are shadows and spots of light. Although they can be marked by a single local interaction, they are incapable of transmitting such mark without further interactions.

Now, there are two main problems with these definitions, which motivated Salmon ultimately abandoning the mark transmission criterion. First, there are two kinds of interactions that are left unexplained (1984, 181-182). Marks occur when at least two processes intersect and separate, but this criterion cannot account for cases of fission and fusion, where the interaction consists in either two processes becoming one or one process becoming two.

y-type: a single process splits in two (for example, radioactive decay, or a hen dividing itself up into a hen and an egg). 
$\lambda$-type: $\quad$ two separate processes merge into one (absorption of a photon by an atom; consumption of a mouse by a snake). ${ }^{10}$

But there is a more serious problem with the mark transmission criterion, for it is not always successful in sorting out causal processes from pseudoprocesses. This can be seen in the following hypothetical case, advanced by Nancy Cartwright, of a pseudoprocess that meets the mark transmission criterion for causal processes (cf. Salmon 1998, 18): if one 'marks' a moving light spot by putting a red patch on the spot of the wall where the light spot is at one instant, and at the same time (or a few nanoseconds before) puts a red filter on the beacon, the light spot would have one characteristic altered by means of a local interaction, and would have continued to carry such characteristic without further local interventions. In such a case, the light spot (a pseudo process) would have met the mark transmission criterion, which is reserved for causal processes only.

Now, the counterfactual character of the mark transmission criterion aims precisely at avoiding this kind of cases. In Cartwright's example, the light spot would have turned red if the single local interaction had not occurred; therefore, this must not be counted as a genuine marking process. A genuine causal process should continue having characteristic $Q$ if the marking interaction had not occurred, but that is not the case here. However, the problem with this answer, as Philip Kitcher has pointed out, is that it places counterfactuals at the core of the mark transmission view, making the allusion to processes and interactions thus defined in principle dispensable (cf. Dowe 2009, 218). This is not a trivial point. As Salmon himself acknowledged, the mark criterion does not explicate what causal processes actually are. It does not show the actual features that make a process causal ([1994] 1998, 252-253). For this reason, along with the first criticism mentioned above, Salmon ended up abandoning the mark transmission criterion ([1994] 1998, 254).

\section{b) Conserved quantity criterion}

Based on Philp Dowe's conserved quantity theory of causation, Salmon redefined causal processes and interactions in a way that overcomes the problems of the mark transmission criterion. He defined causal processes, interactions, and transmission of conserved quantities as follows: Causal interaction: $\quad$ Is an intersection of world-lines that involves exchange of a
conserved quantity.

Causal process: Is a world-line of an object that transmits a nonzero amount of a conserved quantity at each moment of its history (each space time point of its trajectory)

\footnotetext{
${ }^{10}$ In $(1984,182)$ Salmon acknowledged that he was not capable of explicating these interactions with his framework, but he was open to the possibility that the framework may accommodate these interactions after all. In later works he abandoned the mark transmission framework, and so he no longer explored this possibility. As we will see shortly, in the conserved quantity framework these interactions can be easily explicated.
} 
Conserved quantity (CQ): It is any quantity governed by a conservation law (massenergy; linear momentum; charge; etc.)

Transmission of a $C Q$ : $\quad$ A process transmits a conserved quantity between $A$ and $B$ $(A \neq B)$ if it possesses a fixed amount of this quantity at $A$ and $B$ and at every stage of the process between $A$ and $B$ without any interactions in the open interval $(A, B)$ that involve an exchange of that particular conserved quantity. (cf. Dowe 2009, 216-221)

This account of causation defines causal processes by what they actually possess (a conserved quantity), and so it is not counterfactual. It also deals with the problems of fission and fusion interactions in an obvious way: in those cases, the amount of the conserved quantity carried by the processes is either divided or combined $(1998,19-20)$.

\subsection{Scientific Explanation}

Salmon's first account of scientific explanation (e.g. [1975] 1998) proposed the notion of statistical dependence as a way of dealing with the problem of relevance. Given events $A$ and $B$ with probabilities $P(A)$ and $P(B)$ respectively, if the probability $P(A . B)$ is simply $P(A) \times P(B)$ then $A$ and $B$ are statistically independent. If this is not the case, then the two events are statistically relevant to each other. On this view, statistically independent events are completely without explanatory value to one another; on the other hand, statistically relevant events are also explanatorily relevant. Note that this is independent of whether the presence of one event makes the other event expectable. Explanatory relevance is not tied with high inductive probability.

This view, however, is incomplete, for it is not necessarily the case that two statistically relevant events are explanatorily relevant. For example, correlated events that do not have a direct causal connection between them are not explanatorily relevant to each other (otherwise, some of the problems of the DN model would emerge again). Salmon thus proposed a new version of this account, called the Causal-Mechanical (CM) model (e.g. 1984). On this view, it is only when two statistically relevant events are also causally related that one is explanatorily relevant to the other. What is of primary importance is causal relevance, which is not the same as statistical relevance, but a species of it (Salmon 1998, 116).

The crucial idea of the CM model is the notion of the causal structure of the world, which is the network formed by causal processes and interactions. In order to explain the explanandum, the explanation must show how the explanandum fits into this network. Thus, whereas for Hempel to explain was to fit the explanandum 'into the nomic nexus', for Salmon scientific explanations work by showing how the explanandum "fit[s] into the causal nexus" (2006/1999, 120). This is done by tracing spatiotemporally continuous causal processes and interactions that connect the explanandum to its causes (Salmon 1984, 156). Consider the following example.

BAROMETER CASE: A storm (S) was preceded by both a decrease in the reading of a barometer $(B)$ and a decrease in the atmospheric pressure (A). Why did S occur? 
In this case, B and S are statistically relevant to one another, but they are not directly causally connected, and so we cannot appeal to one of them to explain the other ${ }^{11}$. On the other hand, both B and $S$ are causally connected to $A$, and thus we can use $A$ to explain either of them. The idea is that one explains a relation of statistical relevance between two non-contiguous events by invoking a common cause. This common cause is connected to those events by spatiotemporally contiguous causal processes. In this way, we show how the events to be explained 'fit' into the causal nexus of processes and interactions, that is, into the causal structure of the world.

\section{§8. Problems with the CM Model}

Because of its causal character, Salmon's model deals with the asymmetry problem in a natural way. In the flagpole case, it is the height of the flagpole (a causal process) that, (causally) interacting with light waves coming from the Sun (causal processes), explains the fact that there is one section of the floor that is hit by fewer light waves, thus creating a contrast with other sections of the floor that are more illuminated (we describe this contrast - the shadow - in terms of causal processes). However, the model is not too successful on two other fronts.

\subsection{The problem of relevance}

One problem with the CM model, as has been pointed out by Christopher Hitchcock, is that "the nexus of causal processes and interactions as characterized by Salmon is not rich enough to supply the necessary conception of explanatory relevance" $(1995,304)$. James Woodward makes a similar point:

The suggestion that explanation involves 'fitting' an explanandum [E] into a causal nexus does not give us any very precise characterization of what the relationship between $\mathrm{E}$ and other causal processes and interactions must be if information about the latter is to explain E" (Woodward 2003, 351).

The idea of these criticisms is that merely showing how the explanandum fits into the causal network hardly tells us which amongst all causal processes connected to the explanandum are actually relevant for explaining it. It is true that Salmon points out that we do not have to search the whole universe to find out what events bear causal relations to the explanandum, "we have only to examine the interior [of the light cone] and some boundary of some spatial neighborhood of [the explanandum] for a certain time in the immediate past of [it]" (Salmon 1998, 120). But this does not give us instructions about how to narrow down the set of all causes of the explanandum. Take the example of Mr. Jones taking birth control pills. The pills are causal processes that, when ingested, become spatiotemporally connected with the explanandum (Mr. Jones non-pregnancy). Although the pills belong to the causal structure of the world, and are part of the causal nexus into which the explanandum fits, they do not explain the non-pregnancy (cf. Woodward 2003, 352).

\footnotetext{
${ }^{11}$ Note that this is true even though we can predict $\mathrm{S}$ using $\mathrm{B}$.
} 
Now, an answer to this worry may be that whether or not Mr. Jones takes the pills, the probability of him becoming pregnant is zero, and so taking birth-control pills is statistically independent of whether or not Mr. Jones gets pregnant. In general, amongst all causal processes that contribute to the occurrence of the explanandum, only those that really make a statistical difference should be chosen. However, this reply implies that, if we want to know the causal processes in virtue of which those statistical dependencies occur, it is not enough to know the set of actual causal processes and interactions, but which ones, were they or were they not to have been present, would or would not make a difference to the occurrence of the events to be explained. It seems that this counterfactual information is crucial to knowing which actual causal processes are explanatorily relevant. In other words, appealing to the notion of statistical relevance introduces counterfactual reasoning after all.

\subsection{The problem of higher-order explanations}

Here is another problem with the $\mathrm{CM}$ model. Consider the following case due to Frank Jackson and Philip Pettit:

GLASS CONTAINER CASE: The water in a closed glass container reaches boiling temperature -the mean molecular motion is at such and such a level - and the container cracks. Why did it crack? $(1990,110)$.

One common explanation of this case is that an increment in water temperature (say, due to an energy transfer from an external source), increased the internal pressure of the container. The problem is that strictly speaking temperature is not a causal process, but a way of describing the average kinetic energy of the water molecules. A proper CM explanation of this case should appeal to the momentum of the molecules that stroke some molecular bonds in the container's surface. But obviously it is virtually impossible to determine which molecules actually hit the container surface, let alone to back track the molecular interactions that led them to do so. This result is puzzling, for it means that under the $\mathrm{CM}$ model this case cannot be explained. On the other hand, the first explanation - the one that appeals to the increase in temperature- does seem to be a satisfactory one, although it does not accommodate the CM model. We can explain the cracking when we learn that there was an increase in the temperature. How does this explanation work if it is not by tracking specific causal processes?

Jackson \& Pettit call explanations of this kind 'program explanations'. Program explanations do not point to specific causal processes; rather, they appeal to an existential quantification: there will be some set of causal processes that will be efficacious in producing the explanandum.

Jackson \& Pettit distinguish between causally relevant and causally efficacious properties. Causally efficacious properties are those possessed by Salmon's causal processes. They are responsible for the actual occurrence of the explanandum. Causally relevant properties, on the other hand, not only include causally efficacious properties but also higher-order properties: 
$[T]$ he higher-order, inefficacious property is causally relevant to the event produced, because its realization programs for the realization of a lower-order efficacious property and, in the circumstances, for the occurrence of the event in question (Jackson \& Pettit 1990, 114-115; my emphasis).

The crucial concept here is that of 'programming'. In the glass container case, the temperature property is a statistical aggregate, and did not produce the momentum of the molecules that led to the cracking. But the temperature was relevant for the cracking. The instantiation of the temperature property programs or ensures that there will be a set of causal processes (the momentum of a set of water molecules) that will produce the cracking of the container. An explanation that appeals to the temperature gives us important information that helps us to understand why the container cracked.

Moreover, this explanation seems optimal. Even if it was possible to provide a CM explanation for this case (assuming one could back track the trajectory of every single water molecule that hit the container walls -arguably, an impossible deed), the higher-order explanation would be better. The actual molecules that hit the walls seem not to be statistically relevant for the cracking. Had it not been the specific water molecules that hit the container walls, others would have hit it and produced the cracking anyway. This hypothetical CM explanation would miss this important information. Citing the actual trajectory of the particular set of water molecules that hit the container surface is not as important to the explanation as mentioning the property that 'programmed' this to happen. As long as the programming property is instantiated, the cracking would happen anyway, regardless of the actual trajectory of the specific molecules that were efficacious in doing it.

Program explanations are not special or rare cases; in fact, they are all over the place in science: the explanation of a glass that breaks due to its fragility; the squareness of a peg, which ensures that it will not fit into a circular hole; the rabbit that is eaten in a forest infested by a skulk of foxes; etc. Juha Saatsi has pointed out that although Jackson \& Pettit's is not 'a full-blown' theory of explanation, it is "an attempt to make sense of higher-level explanations that do not feature causally efficacious properties" $(2016,16)$. The essential feature of program explanations is that, by appealing to these higher-order properties, they give us modal information about the explanandum that is essential to understand why the explanandum occurred. The CM account cannot explicate why these are satisfactory explanations. As I will show, however, despite the fact that there is an important modal component at play, these explanations can still accommodate the model of ontic accounts of scientific explanation.

\section{§. Woodward's Interventionist Account}

James Woodward's model of scientific explanation is also associated with an account of causation. What is fundamental in causal relationships, according to Woodward, are invariant relationships of dependence between the cause and the effect. For Woodward, a causal relationship is one that

in principle can be used to manipulate the effect by intervening over one of its causes. If systematic interventions on $X$ entail systematic changes on $Y$, then $X$ is one of the causes of $Y$. These dependency relations are at the core of successful scientific explanations. With this account of 
causation, Woodward defines a scientific explanation of $Y$ as an answer to a w-question of the form: what-if-things-had-been-different? These questions can be answered by exploiting the causal relationship between $Y$ and one of its causes (for example $X$ ).

For Woodward, when explaining a physical phenomenon, one must find the right level of description where invariant relationships of dependence hold. Knowing these relationships often requires abstracting away from many of the details of the underlying causal processes that produced the explanandum. On this account, since there are invariant relationships at different levels of description, causal relationships can be found at each of these levels, even when the underlying causal processes cannot be tracked down. As we will see, this is why Woodward's is an ontic account of scientific explanation. These invariant relations are discovered when the system is described at the appropriate level. But the invariances are objective; they do not depend, for their existence, on the person who describes them.

Contrary to Salmon's, Woodward's account of causation and scientific explanation does not depend on tracking down spatiotemporally continuous relations between the events in the explanans and the explanandum. In what follows, I discuss the notions of causation, intervention, and invariance in more detail, and then focus on Woodward's account of scientific explanation.

\subsection{Causation}

For Woodward $X$ is a cause of $Y$ if there is an invariant relationship of dependence between $X$ and $Y$ that can be used for manipulating or controlling $Y(2003,14)$. These invariant relationships are objective features of the world. If systematic interventions over $X$ entail changes in $Y$ (or in the probability of $Y$ ), then $X$ is a cause of $Y$. This dependence between $X$ and $Y$ must be invariant under a range of interventions.

Just as does Salmon's, Woodward's notion of causation allows distinguishing between two variables that are simply correlated and two that are causally connected. In the barometer case, for example, a decrease in the reading of a barometer (B) is almost always followed by a storm (S). However, it is not possible to control whether or not S occurs by intervening over B. If one changes the reading of the barometer by means that affect such a reading only (say, by locally cooling down a mercury barometer), obviously the occurrence of the storm would not be affected, and the correlation between B and S would break down. On the other hand, when the atmospheric pressure $\mathrm{A}$ drops, it is more likely ${ }^{12}$ that $\mathrm{S}$ will occur. This is a causal relationship not only because without a decrease in $\mathrm{A}, \mathrm{S}$ would not have been more likely to occur, but also because within some range, gradual changes in A would systematically alter the probabilities of $\mathrm{S}$.

\footnotetext{
${ }^{12}$ For Woodward, being 'more likely' to occur does not mean actually being likely to occur. Just like with Salmon's account, a factor is a cause if it affects the probability of its effect, regardless of whether it conveys high probability to the occurrence of the effect.
} 


\subsection{Interventions}

An intervention I is "an idealized experimental manipulation carried out on some variable $X$ for the purpose of ascertaining whether changes in $X$ are causally related to changes in some other variable Y" (Woodward 2003, 94). This notion of intervention is in itself causal, so there is a sense in which Woodward's account commits a circularity. However, as he points out, this is not a vicious circularity. It is true that the concept of causation is not fully defined within his view, because it does not "yield a reduction of causal talk to non-causal talk" (Woodward 2013), and in that sense the definition is circular; but his is an account of causal relationships between two or more events or processes. The criteria for $/$ to be a proper intervention over $X$ (in order to manipulate $Y$ ) does not include any reference to the causal relationship between $X$ and $Y$. So, the characterization of interventions is not viciously circular because it does not presuppose "that we already have causal information about the very relationship that we are trying to characterize" (Woodward 2013). ${ }^{13}$

Another feature of interventions is that they should be 'surgical', that is, they should affect one of the variables without directly affecting the other one. ${ }^{14}$ For example, consider a pendulum with a copper wire of length $L$ that has a period $T$, as described by:

$$
T=\sqrt{\frac{L}{2 \pi}}
$$

Because interventions over $L$ would produce systematic changes in $T, L$ is a cause of $T$. One can intervene over $L$, say, by dilating the wire with heat. But not every way of heating it up would count as an intervention. If we pump hot air in the room this would increase the wire's temperature, producing the dilatation, but the air may also push the wire sideways, thus directly affecting the period $\mathrm{T}$. This disqualifies it as an intervention in Woodward's sense. A proper intervention may be, for example, to stretch the wire.

Finally, it is important to note that, in many senses, interventions need not be possible. For starters, they do not need to be humanly or technologically possible. Interventions can be the result of natural events. Consider, for example, the relationship between the moon and the tides. That the moon causally affects the tides can be seen if we take the natural rotation of the moon around the earth as a 'natural' intervention. Gradual changes in the position of the moon entail changes in the tides, due to the gravitational influence of the moon over the ocean water.

Interventions, however, need not even be physically possible, let alone have actually occurred. For example, changing the moon's orbit would also count as an intervention. But when

\footnotetext{
${ }^{13}$ This discussion of interventions is important for one of the main points I make in this dissertation. Woodward defined interventions causally, in order to find out causal relationships. But it is also possible to conceive noncausal interventions (I will call them 'deformations'), which would yield information about noncausal relationships of dependence. As I will argue below (section §18), there are scientific explanations that require mentioning these noncausal relationships.

${ }^{14}$ To draw an analogy with experimentation, we could say that one must manipulate the independent variable in order to assess changes in the dependent variable.
} 
we think of the conditions under which this may happen, the situation gets complicated (cf. Woodward 2009, 255). If such alterations were produced, say, because the moon is attracted by another planet, such a planet would also affect the tides directly, and therefore the alteration would not be an intervention because it would not be surgical. It is difficult to imagine a causal intervention that affects only the moon's orbit.

In spite of this, we can still think, hypothetically, of what would happen if the orbit were to change, or the size of the moon were to be doubled, etc., even if it may not be physically possible to carry out these interventions. So, even if it is true that there is no physical way for these things to occur, we still believe that the size of the moon, and its orbit, are causes of the tides. Why? In this case, because of our reliance on Newtonian mechanics. Identifying causal relationships requires analyzing a series of counterfactual claims. We can answer these counterfactuals, for example, by analyzing and manipulating the mathematical model that describes the physical system in question. In this particular case, the model consists in Newtonian laws of movement and gravitation, as well as the geometrical laws of the composition of forces. Without actually intervening over the physical system, we can analyze the truth of counterfactual claims by manipulating such equations, even though it is physically impossible for the scenarios that correspond to those manipulations to occur. This manipulation of the mathematical model does not imply that the mathematical or logical relationships in the model are actually carrying out the explanatory work in the explanation. As I will emphasize in further sections (see especially $\S 14$ and $\S 15$ ), they are the physical features represented by the model that explain the explanandum (in this case, the tides).

It is important to note that, on this account, interventions are performed (or thought of) in order to find out causal relationships. Altering the actual mechanism that relates the cause and the effect would not count as a proper intervention. The idea is that the actual mechanism must remain untouched. Untouched mechanism means that the relation between the cause and the effect should remain invariant. This will become clearer in the next subsection.

\subsection{Invariance}

We saw that for Woodward causation is about objective relationships of dependence that occur in the world (that is why this is an ontic account of explanation). These relationships are invariant under interventions, but not all interventions would preserve this invariance. Consider the case of a spring that obeys Hooke's law, which is a law that describes the behavior of springs when elongated by an external force. Depending on the elongation of the spring, it would pull with a restoring force given by the following equation:

$$
F_{r}=-K \Delta L
$$

In this equation, $K$ is the constant that describes the stiffness of the spring, $F_{r}$ is the restoring force that the spring produces in a direction opposite to the elongation, and $\Delta \mathrm{L}$ is the difference between the initial length $L_{0}$ and the elongated length $L$. A cause of the magnitude of the restoring force $F_{r}$ is the elongation $\Delta \mathrm{L}$ of the spring. Systematic variations in $\Delta \mathrm{L}$ would produce systematic changes in $F_{r}$. In that sense, the relation between $\Delta L$ and $F_{r}$ is invariant. However, not all possible 
values of $\Delta L$ will preserve this invariance. When a spring is stretched beyond its elastic limit, it loses its capacity of producing a restoring force. It is only within some parameters that interventions over the elongation of the spring will preserve the invariant relationship between $\Delta L$ and $F_{r}$. These parameters are called the range of invariance. Beyond this range the mechanism is altered and the invariant relationship breaks down. Within the limits of this range, however, the elongation is a cause of the restoring force, as it is described by Hooke's law. Beyond this range there is no such causal relationship.

The idea, then, is that some dependency relationships remain invariant under a wider range of interventions (for example, the gravitational attraction between two bodies), whereas others have a limited range (for example Hooke's law). The larger the range of the invariant relationship, the greater the amount of counterfactual cases that could be analyzed by appealing to it, and so the greater the capacity to answer w-questions. As we will see, since answering these questions is fundamental to scientific explanation, the larger the range of invariance the greater the explanatory power of a generalization.

\subsection{Scientific Explanation}

Woodward's model of scientific explanation is called the Interventionist model. According to him, a scientific explanation should answer w-questions of the form: what-if-things-had-beendifferent? $(2003,11)$. On this account, scientific explanations should inform us about what would have happened to the explanandum if the facts described in the explanans had been different, and in that way we learn about the actual facts that made a difference to the occurrence of the explanandum. This is done by exploiting invariant relations of dependence in the world.

In the bouncing ball case, for example, we can control the bouncing height by intervening over the initial height. Within some range, the relationship between initial and final height is invariant under interventions. But of course, the range under which this invariant relationship holds is not too wide. If the ball was dropped from $100 \mathrm{~m}$ height (in a vacuum) it would probably smash into pieces when it hit the floor. Due to this limited range, explaining the $26,8 \mathrm{~cm}$ bouncing height by appealing to the initial height alone is not a fully satisfactory explanation. A better explanation would be one that appeals to the relation of semi-elastic collision between the floor (say, made out of concrete) and the ping pong ball (made out of plastic celluloid). One equation of semi elastic collisions is:

$$
V^{\prime}=\rho V
$$

The coefficient $\rho$ depends upon some characteristics of the colliding objects, in this case the thickness of the ball, the elastic properties of the celluloid plastic, the rigidity of the floor, etc. This relationship is stable under a wider range of interventions than the relationship between initial and final height. By intervening over those characteristics, we can change the value of $\rho$, and we can answer a greater number of w-questions about the final height.

Now, it may appear that the more detail we introduce in our analysis the better the explanation would be. But, as we have already seen in section $\S 8$ with those cases that require 
program explanations, this is not always so. These cases can be well understood under Woodward's account. Consider the following program explanation:

THE RABBIT CASE: A rabbit is let loose in a forest that has recently been infested by a skulk of foxes. Given its average speed, it would have taken the rabbit 2 minutes to run away and leave the forest. But in the end, the rabbit was eaten by a fox. Why did this happen?

There are some specific characteristics of foxes and rabbits that may explain this, for example, that foxes are carnivorous, faster and stronger than rabbits, aggressive; etc. These features, along with specific details of the trajectory of the fox that ate the rabbit and the rabbit itself, explain why the rabbit was eaten by a particular fox. But there is a sense in which the specific trajectories of the fox and the rabbit are not relevant for explaining the explanandum. There were so many foxes in the area that, had it not been that particular fox, another fox would have probably eaten the rabbit before he managed to escape. If we want to explain why the rabbit did not make it, the fact that it was that specific fox is irrelevant. As we saw, this represents a problem for Salmon's account, where causal explanations should track down the trajectories of spatiotemporally continuous causal processes. For Salmon, only causal processes and their interactions should be cited in causal explanations. Since only individual foxes are causal processes, only they should be mentioned. But as we saw, by doing this the explanation would omit important modal information about the explanandum.

The key point here is that whether or not a given explanation requires a program explanation depends on the specific explanandum that is to be explained. In Woodward's view, in order to acquire the information needed to answer w-questions we must find the right level of description. In this specific case, given the explanandum, we must describe this situation at the level of the aggregate of foxes, and not at the level of individuals. What happened with one specific fox is statistically irrelevant to the occurrence of the event described by this explanandum. It was the increment in the number of foxes in the area that increased the probabilities of an attack. We can intervene over this by analyzing a similar case in which the rabbit is placed in forests with a different number of foxes. If there are fewer, the probabilities of the attack would be reduced, and they would gradually increase if the number of foxes increases. This satisfies Woodward's condition for invariance. Because the relationship between the number of foxes and the probabilities is invariant (we can control the probability of being eaten by causally intervening in the number of foxes), these two elements hold a causal relationship. This causal relationship can be used to answer a range of w-questions, and so we can explain the occurrence of the explanandum by appealing to the number of foxes (a higher-order property of the aggregate).

The conclusion we get from this example is that there are invariant dependency relationships - that is causal relationships - at higher levels, and that in many cases these higherorder causal relationships are more explanatory than the correspondent lower-level ones. If we want to scientifically explain a given phenomenon, we should find the level at which these invariant relationships of dependence are stronger, because this would allow us to answer a wider range of w-questions. Moreover, despite the fact that it is our choice to describe the situation at the level of the aggregate of foxes, rather than at the level of the particular fox that ate the rabbit, 
this explanation relies on the fact that the probability of the rabbit being eaten was higher than it would have been had the rabbit been in a forest with fewer foxes. This higher probability is an objective fact of the situation that is discovered once the situation is described at the right level.

This description-relative approach is compatible with an ontic approach to explanation. As we saw, in an ontic conception:

The aim of scientific explanation... is to fit the event-to-be-explained into a discernible pattern. This pattern is constituted by regularities in nature (Salmon 1984, 121).

In Woodward's model, the discernible patterns into which the explanandum fits are the objective invariant relationships of dependence, some of which may qualify as laws. These invariant relationships can be found when the system to be explained is described at the right level of description, but their existence itself does not depend upon those pragmatic considerations. As Woodward puts it:

[W] hat matters for purposes of causal explanation is what the real dependency relations in the world actually are: what would in fact happen to some potential explanandum if other conditions were changed in various ways $(2003,202)$.

When we appeal to 'the number of foxes' as a property of the aggregate, we are capturing an objective relationship between the aggregate and the probability of the rabbit being eaten. Under a certain range, this relationship is invariant. It is not description-relative that a rabbit in a forest with one hundred foxes is more likely to be eaten before he managed to escape than if he had been in a forest with two foxes. It is this objective relationship that is fundamental in explanations, and for that reason, Woodward's account is ontic.

It is important to note that, from this point of view, invariant relationships are explanatorily more fundamental than laws. We saw that, for the DN model, appealing to laws of nature was essential for scientifically explaining a physical phenomenon. However, invariant relationships with a limited range may not qualify as genuine laws of nature, but still they have explanatory power. This is because they allow us to answer an (albeit limited) range of counterfactual questions. Thus, from this point of view, the laws of nature are important for scientific explanations only insofar as they describe invariant relationships.

Woodward's model successfully deals with the problems we have seen in this chapter. Since it is causal, it does not have the problem of asymmetry. In the flagpole case, for example, if we keep the other conditions fixed, like the position of the Sun and the angle between the flagpole and the floor, we can control the length of the shadow by intervening over the height of the flagpole. There is no way of doing the opposite: if we keep other conditions fixed, we cannot control the height of the flagpole by manipulating the length of the shadow. ${ }^{15}$ In the same way, Woodward's model also deals easily with the problem of relevance. Whether or not Mr. Jones takes birth control pills does not make any difference in whether he gets pregnant. There is no

\footnotetext{
${ }^{15}$ This rules out Van Fraassen's counterexample of section $\S 5$.
} 
way of controlling the possibility of getting pregnant by intervening in whether or not he takes the pills. If we want to explain why he did not get pregnant despite the fact that he has been having intercourse regularly, we need to find some factor that, had it been different, it would have contributed to the pregnancy (Woodward 2003, 352). One such factor may be gender. If he had been female, there may have been some probabilities of getting pregnant. One way of controlling this may be by a randomized control trial over a sample of males and females. We can improve this explanation by appealing to physiological and anatomical factors.

Woodward's model also accounts for higher-order explanations, as we have just seen in the rabbit case. In the glass container case, the increment in kinetic energy produced an increment in the container's internal pressure. For Woodward, this relationship between temperature (average kinetic energy) and pressure is causal because we can control the pressure by intervening over the temperature (the intervention in this case could be an energy transfer from an external source). Describing this case at the level of the aggregate allows us to answer a wider range of wquestions than if we describe it at the level of the actual water molecules that caused the cracking, due to the fact that the outcome is modally stronger than what could have been rendered by appealing to that particular set of water molecules.

\section{$\S 10$. Conclusion}

According to Woodward's model, what is fundamental in scientific explanation is information about objective relationships of dependence that can be used to answer counterfactual questions. My goal in the remainder of this dissertation is to show that, for scientific explanation, emphasis on the notion of counterfactual information subordinates the notion of causation. In other words, if interventions are conceived more broadly, as modifications over one of the relata, they can be used to provide this counterfactual information, leaving causal interventions as special cases. Given the importance of this counterfactual information to scientific explanation, the conceptual space is open for an ontic account of noncausal scientific explanations. 


\section{CHAPTER 2. Mathematical Models in Science}

\section{$\S 11$. Introduction}

This chapter focuses on the role of mathematical models in scientific representation and scientific explanation. The discussion of this chapter provides the framework for my account of Mathematical Explanations of Physical Phenomena, which is the focus of chapters 3 and 4.

I begin this chapter presenting the notion of mathematical model, including a conceptual distinction between abstraction, approximation, and idealization (§12). I also discuss the different kinds of models there are, based on the representational ideals of the scientists who use them. I next analyze two accounts of the application of mathematics: Christopher Pincock's mapping account (§13), which highlights the importance of the structural resemblance between the model and the target system; and Bueno, Colyvan, and French's Inferential Conception (§14), which, in addition, emphasizes the context of application, as well as the role of the interpretation the users of the model assign to its mathematical structures. The main point here is that, without a proper interpretation, mathematics says little about the physical world. Next, I use the Inferential Conception to clarify the role of mathematics in scientific explanation (§15), and discuss a criticism by Robert Batterman (2010). I finish the chapter by presenting a possible shortcoming of the Inferential Conception, which is that this view does not distinguish between explanatory and nonexplanatory mathematical derivations.

\section{§12. Abstraction and Idealization in Mathematical Modeling}

\subsection{Mathematical Models}

In this section I introduce Michael's Weisberg's notion of mathematical models as interpreted mathematical structures. This view is compatible with the two accounts of the applicability of mathematics I discuss later in this chapter (sections $\S 13$ and §14). As we will see, this view is not committed to a specific account of the ontology of mathematical objects. The only requirement is that it has to be one in which it makes sense to talk about mathematical objects $(2013,29)$.

Weisberg defines scientific modeling as the indirect study of real world systems via the construction and analysis of scientific models (Weisberg 2013,4). A model is a structure that has been assigned a given interpretation, so that parts of the structure correspond to a given target. Weisberg distinguishes between concrete models (also called 'material' or 'physical') and mathematical models. ${ }^{16}$

Concrete models are interpreted physical structures, like, for example, a laboratory rat intended to represent an aspect of a human organism, a scale model of a terrain built in a subfreezing lab, or the hydraulic scale model of the San Francisco Bay built in 1957 in a warehouse in Sausalito (Weisberg 2013, 3). These physical objects have properties that are missing in the

${ }^{16}$ He also talks about computational models but these are not important for the purposes of this dissertation. I explain what concrete models are only to highlight, by contrast, some aspects of mathematical models. 
target, and also lack some properties that their targets have, but they become scientific models when some of their structural features are interpreted in terms of a given target system (which can be another physical object or a class of physical objects). In concrete modeling, one has direct access to the model under study. For example, before administering a new drug to humans, researchers often observe the effects of this drug in animals, in what is called the 'pre-clinical' stage of research. Researchers interpret some parts of the animal's organism as representing a human organism in virtue of some sort of similarity between the two. In the same way, a modeler may assume that the slope of her model corresponds to the actual terrain that is the target of the model (this assumption is false, but for some purposes the distinctions between the two may be negligible - this depends on the fidelity standards of the modeler). Finally, the Army Corps of Engineers studied the possible effects of John Reber's plan of building two dams in the San Francisco Bay by manipulating and observing the scale model, and in that way, they concluded that the implementation of the Reber plan would have had negative consequences for the bay (Weisberg 2013, 3).

Mathematical models, on the other hand, are mathematical structures that have been assigned an interpretation $(2013,15)$. Examples of mathematical models are roads with frictionless slopes, infinitely deep oceans, or the Lotka-Volterra model of the relation between population and prey. These models cannot be assessed or measured directly, so the way researchers manipulate and learn about them is by manipulating the equations, graphs, etc. that describe them.

Now, mathematical models are often confused with their descriptions (i.e. the actual graphs, equations, etc.), but the fact is that they are not their descriptions, first because there are many ways of describing the same mathematical structure used in a model; but also, as Weisberg has pointed out, because very often models have properties not mentioned in the description, and sometimes the description itself needs to be interpreted in order to capture the right model. In the Lotka-Volterra model, for example, the Lotka-Volterra equations cannot be assigned negative or irrational values $(2013,40)$, otherwise they would not be describing predators and preys. As Weisberg puts it:

A modeler often conceives of a model in a vague way, writes down some equations to describe the model she thought she had in mind, studies the model actually specified by the equations, and determines whether or not they pick out the right model. Situations can arise where the modeler's imagination picks out some set of models and her model description picks out a different set of models, necessitating a refinement either to her imagination or to her model description (2007b, 217).

The idea is that mathematical models are picked out by their descriptions, but they are not themselves their descriptions, even though it is often the case that one can only access the details of the model by analyzing the description itself. 


\subsection{Abstractions, Idealizations, and Approximations}

Mathematical models can be conceived with no intention of representing empirical systems, but most models used in science are conceived with a specific target in mind. ${ }^{17}$ Models intended to represent concrete targets are constructed by a process of abstraction and/or idealization, and most of the time they represent their target systems only approximately. The use of these terms, however, varies considerably throughout the literature, so let me make explicit what I mean by them.

An approximation is an inexact representation of a feature of a target system. Approximations can be included in a model because of practical limitations in approaching a system (for example, because the measurement instruments lack precision), or for tractability purposes (to round up things in the mathematical description - i.e. 6,1 rather than 6,0972).

I will call abstraction, as a process, the mental activity of focusing on certain features of a system, deliberately ignoring others. The outcome of the process of abstraction is a system whose properties are intended to be direct representations of real properties. For example, the LotkaVolterra model focused on the fact that predators eat prey (and not the other way around), but not on, say, the size of these animals. Abstraction, as a noun, is the outcome of this process of mentally stripping from a system some of its features while retaining others (for example, representing a person's weight with a number, ignoring other aspects such as her height, etc.). Crucially, an abstraction in a model will be a feature of the model that is intended to have a direct correlate in a real system. This sense of abstraction (process and noun) is often called 'Aristotelian abstraction'.

Here it is important to mark distance from another sense of the word abstraction. Mathematical models are often said to be abstract in a Platonic sense (as opposed to concrete), but not necessarily because mathematical models are built up out of properties abstracted from physical systems. ${ }^{18} \mathrm{~A}$ mathematical sphere can be used to represent the Sun; this does not necessarily mean that mathematical 'sphericity' is an actual feature of the Sun, or that the Sun's shape is an instance or an exemplification of a mathematical sphere in a strong metaphysical sense (like, for example, in Shapiro 1997) (more on this below).

Finally, idealization, as a process, is the mental activity of constructing a system, some of whose properties are intended to be direct representations of properties of the target system, but

\footnotetext{
${ }^{17}$ An exception is the case of models of three-sex species used in biology. Although these models have not been conceived with a real target in mind (there are no such three-sex species), their usefulness in science derives from the fact that they can help us understand why there are no such three-sex species (see Weisberg 2013, 132).

${ }^{18}$ See Cargile 2003, 147. Contrary to this, some mathematical structuralists argue that this is in fact the correct description of the relationship between mathematical structures and concrete structures. Michael Resnik (1997), for example, conceives physical patterns as mathematical structures of physical systems, and Stewart Shapiro (1997) argues that mathematical structures can be exemplified by concrete structures. Pincock (2015) holds a similar view. In any case, what I am doing here is a conceptual distinction between these two senses of abstraction.
} 
that also has desirable properties the target system lacks. In that sense, an idealization, as a noun, is a property of a model that does not directly represent properties of the target system. Insofar as the outcome of an idealization process also has properties that are direct representations of some properties of the target, the process of idealization can also involve a process of Aristotelian abstraction. But the crucial point of the process of idealization is that the outcome is a system that includes elements that have been either intentionally distorted from the target or that have been made up, in a way that they do not have a direct correlate in the target at all. ${ }^{19}$ Some authors call the former idealizations proper, and the latter outright fictions, but this distinction will not be relevant for my purposes. Both distortions and fictions will count as idealizations for me.

Abstraction is always present in modeling, in the sense that the modeler has to choose a target by focusing on some aspects of the world and ignoring others (Weisberg 2007b, 228). But the models themselves combine approximation, abstraction and idealization. Consider for example the case of modelling a real slope with a frictionless slope. The imagined slope includes properties such as inclination, surface length, etc. and ignores other properties such as surface width, color, etc. In that regard, the model is the outcome of a process of abstraction. In addition, it has a plane surface, which is a distortion of the real surface, and it has the desirable property of being frictionless. So, this is clearly an idealization. Finally, the total length of the real slope is only approximately represented by the model, the accuracy criteria of this approximation depending on the practical interests of the scientists given the situation at hand. In the case of the angle of the real slope, things are more complicated from a philosophical perspective. Strictly speaking, the slope does not have an angle. An angle is the arc formed by two rays or two planes with common origin (a point or a line). We cannot say that the 'physical' angle is approximately represented by the mathematical angle in the model because there is no such thing as a concrete (mathematical) angle. More properly, we should say that the mathematical angle is an idealization obtained from considering a property abstracted from the target, namely, the inclination of the slope. The angle and the inclination of the slope may have some things in common, and we can learn things about the latter by examining the former, but the angle has not been abstracted from the target.

The example above illustrates that, although it is possible to conceptually distinguish between idealization and abstraction, in the case of mathematical properties it is often hard to separate the two. Paul Humphreys has pointed out that almost all attempts to abstract mathematical properties from the physical world collapse into idealization (cf. 1995, 159), because they almost always end up falsifying the properties of the physical system; this is because the model is using "the simple and precise concepts of mathematics to represent complex and imperfect real properties" $(1995,158)$. For example, representing a concrete circular shape as a mathematical circumference is an idealization, since no concrete circular shape has the features of a perfect circle (see Humphreys 1995, 158). The same happens, as we have seen, when trying to abstract a mathematical angle from a real slope. Although focusing on some particular aspects of an object rather than others is a form of abstraction, the representational mathematical

\footnotetext{
${ }^{19}$ In both cases it is assumed that the modeler is aware of these misrepresentations.
} 
property is an idealization. In that sense, mathematical properties are not (Aristotelian) abstractions obtained from concrete properties.

However, although it is hard to separate abstraction and idealization in the case of mathematical properties, there are some mathematical models based solely on abstractions, without incorporating idealizations, like, for example, some applications of graph theory. Consider a model intended to represent 'hand shakings' in a group of people. Imagine that there are ten persons in a room shaking hands with each other, and that we want to represent this situation mathematically because, say, we want to calculate the total number of hand shakings. In order to do this, we must abstract away from the particularities of the people involved, and the details of every particular hand shaking. The only features relevant to representing this situation are the number of people and the hand shakings (i.e. the 'connections') between every pair of them. For that reason, we can use a graph to represent this case. For this particular situation, what we need is a complete graph. A complete graph is one that has edges connecting every pair of vertices. The representative model can use the following description:

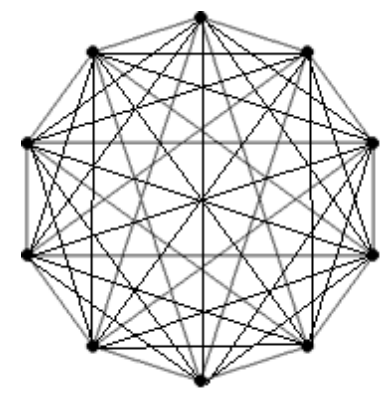

fig. 1

Complete graph with 10 vertices

Here only 'number of people' (10) and total hand shakings (45) are represented, both real properties, and arguably there are no additional elements such as idealizations. So, this seems to be a case of a mathematical model based solely on abstractions, without including idealizations (in the sense defined above). This does not necessarily mean, however, that the graph is representing the mathematical structure of the system of people (as, for example, Shapiro 1997 and Pincock 2015 would suggest). The graph is a mathematical representation of the salient physical features of the situation, given the problem at hand. As I will argue in sections $\S 13$ and $\S 30$, in order to account for the usefulness of mathematics in science it is not necessary to assume that physical systems instantiate mathematical properties. 


\subsection{Galilean and Minimal Mathematical Models ${ }^{20}$}

The use of mathematical models varies depending on the representational ideals of the scientists and the scientific community, and on the details of the situation at hand. Weisberg distinguishes between Galilean modeling practices and minimal modeling practices. Sometimes a model is used with a Galilean interest in mind, that is to say, the abstractions and idealizations are incorporated in the model with the purpose of simplifying it in order to make it mathematically tractable. The idea is to start with a simple model and then add other relevant factors in order to increase accuracy. For example, if we want to explain why a certain body takes some time to slide down a slope, we could start with a frictionless slope as a model, the mathematical specification of which, with respect to the problem at hand, is very simple. When more information is available, or when our computational powers increase, we would complicate the model, adding, say, a static friction coefficient, then one type of dynamic friction, then two, until we are able to assign a friction function to each point, thus accounting more accurately for the body's movement. The reason why these simplifications are introduced is basically pragmatic. The ultimate goal of introducing these models is to simplify the representation, but only until one is capable of getting rid of all the inaccuracies. These models are called Galilean because it was the modeling practice followed by Galileo Galilei (cf. McMullin 1985; Weisberg 2007a; 2013).

In other models, however, the abstractions and idealizations play an essential role in increasing the model's representational power. These are called minimal models. Minimal models are those that include the core relevant features of the target, given a modeling situation (2013, 100). As opposed to Galilean models, the leaving out of details is not done for increasing tractability. Here it is not the goal of the modeler to progressively include back those excluded details. In many cases the features of interest represented by the model may be shared by other targets as well. In those cases, a complication of the model would make it tailored to only one specific target, losing the model's generality, which could be an advantage. This is the case, for example, of the graph representation of the ten people interchanging handshakes, which can be used to model connections between a vast domain of objects, not only the ten people of the example. Another such a case is the use of the Poisson model to represent phenomena as varied as organisms per unit volume in a dilute solution, telephone calls, cars, radioactive decay, chromosomes, flying bombs, caught fish, etc. (see Humphreys 2004, 88-91 for details). As we will see ( $\$ 28$ and §29), this generality is useful for highlighting the modal strength of some developments of the systems, independently of many initial conditions. The model shows that any system that has the relevant minimal features will behave similarly.

Minimal modeling is also useful when the idealized components of the model are essential for highlighting relevant properties of the target. In those cases, although the models are false in

\footnotetext{
${ }^{20}$ This section relies on Weisberg (2007a) and (2013). However, Weisberg distinguishes between Galilean and Minimal Model idealizations. In my discussion, I extend the notions from 'Galilean' and 'Minimal' idealizations to models in general, including those that do not contain idealizations. I have shown that at least some graph theoretical models do not contain idealizations (e.g. the hand-shaking case), and yet, as we will see, they can be understood as either Galilean or minimal models.
} 
some respects - because they have properties that do not directly represent features of the target system - these falsehoods play a representational role (albeit an indirect one). Take, for example, the case of the behavior of low pressure gases. If we want to represent the relationship between pressure and volume, as described by Boyle's law, we can construct a model where collisions between gas particles are perfectly elastic. Although this element does not directly represent what is going on in the real gas, it indirectly contributes to highlight the fact that the energy lost in individual particle collisions is unimportant for representing the relationship between these two properties. This highlighting role is an indirect representational role. Another such a case, that I will discuss below in $\S 15$, is the ray representation of light, used to explain features of the rainbow. Although light beams are not rays, the ray representation highlights the fact that when the ratio between wave length $(\lambda)$ and the radius $(r)$ of water drops falls below a certain threshold, the wave features of light are unimportant for explaining the rainbow's angle of incidence with respect to the direction of the Sun.

Now, whether a model is Galilean or minimal is not a feature of the model itself. It is often the case that the same model can be considered from a Galilean interest or a minimal modeling interest in mind, depending on the representational ideals according to which the model has been constructed or will be used. For example, one can use a straight segment to represent the length of a road, either with the goal of eventually improving this representation so as to accurately capture the actual shape of the road (Galilean interest), or because by using this representation one can, say, measure the total elevation of the final point with respect to the initial one (minimal model interest). The intended interpretation of some aspects of the model, as well as the uses to which the model is put, are essential to determine whether it is Galilean or minimal.

\section{$\S 13$. Pincock's Mapping Account of the Applicability of Mathematics}

When using a concrete model as a representation of another system, we assume that the model and the target are similar in some relevant respect. For example, a rat's organism shares some similarities with human organisms when interacting with certain drugs, and because of that we can infer things about human reactions to those drugs by studying rats. In the same way, the San Francisco Bay model shares some relevant causal relations with the actual bay, like the fact that the interactions between the sea bed and sea water flow are similar to the ones between the bottom of the model (embedded for that purpose with thousands of copper strips) and the water (see Weisberg 2013, 8-10 for details).

But how can a mathematical model, being abstract (as opposed to concrete; see 12.2 above), be used as a representation of a concrete system? Mapping or structural accounts of the applicability of mathematics hold that mathematical models capture structural features of the concrete phenomenon. The basic idea is that there is a correspondence (specifically, a mapping) from mathematical structures in the model to physical structures. It has often been emphasized (e.g. Suárez 2010), however, that structural similarity is not enough for representation. Using one structure to represent another requires some context dependent factors such as the intended interpretation of the scientists doing the representing. So, in addition to structural relations, a full 
account of mathematical representation must include these intrinsically pragmatic issues at the core of the application process.

In order to understand Pincock's account, we need first to define the notions of structure and structural relations, as well as the notion of partial structure. These notions will also be useful for understanding the inferential conception of the applicability of mathematics (Section $\S 14$ ).

\subsection{Structural Relations and Partial Structures}

\section{a) Structure and Structural Relations}

The notions of structure and structural relation are straightforward. A structure is the formal network describing the set of relations that obtains between a set of objects. A structural relation between two systems is one that obtains in virtue of their respective formal networks. As Pincock puts it:

A structural relation is one that obtains between systems S1 and S2 solely in virtue of the formal network of the relations that obtain between the constituents of S1 and the formal network of the relations that obtain between the constituents of S2... [where a] formal network is a network that can be correctly described without mentioning the specific relations which make up the network (Pincock 2012, 27).

A structural relation can be some kind of isomorphism or homomorphism. An isomorphism between structures $S$ and $S^{\prime}$ is a one-to-one structure-preserving correspondence or mapping between all of the elements of their respective domains and all the relations between them. $A$ homomorphism is also a correspondence of these features, but it is not necessarily one-to-one. In the case of applied mathematics, the basic idea is that if we use a mathematical structure $M$ to represent a physical system $P$ (say, we want to explain something about $P$ ), a substructure $M_{1}$ of $\mathrm{M}$ must be homomorphic (or isomorphic) to some relevant substructure $\mathrm{P}_{1}$ of $\mathrm{P}$.

Let us see this with an example due to James Robert Brown (2008). Consider the case of a homomorphism between the relation of physical combination of weight, and the mathematical operation of addition:

Physical domain D: [e.g. bodies $a, b, c, \ldots] ; u$ is the standard unit

Physical relations $R_{1}, R_{2}, \ldots R_{n}$ [' $\leq^{\prime}$ ': weights the same or less than; ' $\oplus$ ': physical combination]

Physical structure $P=\langle D, \leq, \oplus, u\rangle$

Mathematical domain $D^{*}$ : [e.g. $\mathrm{R}^{+}$]

Mathematical relations $\mathrm{R}^{*}, \mathrm{R}^{*}{ }_{2}, \ldots \mathrm{R}^{*}{ }_{\mathrm{n}}[\leq ;+]$

Mathematical Structure $M=\left\langle R^{+}, \leq,+, 1\right\rangle$ 
Homomorphism $\varphi: \mathrm{D} \rightarrow \mathrm{R}^{+}$

Three conditions:

$$
\begin{aligned}
& \text { (1) } a \leq b \rightarrow \varphi(a) \leq \varphi(b) \\
& \text { (2) } \varphi(a \oplus b)=\varphi(a)+\varphi(b) \\
& \text { (3) } \varphi(u)=1
\end{aligned}
$$

In this case we would say that the mathematical model $\mathrm{M}$ stands in a structural relation of homomorphism with respect to the physical system P (cf. Brown 2008, 52).

\section{b) Partial Structures}

Two systems can be structurally similar with respect to some aspects, without being structurally similar with respect to others. And in fact, they can be similar in some known aspects even if one is not sure whether they are similar with respect to others. In the case of applied mathematics, what we usually find is that there are some relations in the model isomorphic (or homomorphic) to the target, some other relations that we know not to have any correspondence at all in the target, and yet some third set of relations that we do not know whether or not obtain in the target. So rather than a full isomorphism (homomorphism), what is often the case are partial relations between physical and mathematical structures. Newton da Costa and Steven French advanced an account of partial structures, which accommodates the uncontroversial fact that most of the time our knowledge of the empirical domain is incomplete, and that mathematical models often have more structure than their targets. In da Costa \& French (2003), for example, they define a partial structure in the following terms:

Consider a binary relation $R$, which can be introduced as follows: $R$ is an ordered triple $<R_{1}$, $R_{2}, R_{3}>$, where $R_{1}, R_{2}$, and $R_{3}$ are mutually disjoint sets such that $R_{1} \cup R_{2} \cup R_{3}=A \ldots R_{1}$ is the set of ordered pairs which are satisfied by those sentences expressing the relationships between the entities concerned, $R_{2}$ is the set of ordered pairs not satisfied by these sentences, and $R_{3}$ is the set of ordered pairs for which it is left open whether they are satisfied. When $R_{3}$ is empty, $R$ constitutes a normal binary relation and can be identified with $R_{1}$ (da Costa \& French 2003, 19).

In other words, $R_{1}$ and $R_{2}$ are relationships in the model that have been assigned an empirical interpretation. Relations pertaining to $R_{1}$ are those that have been interpreted as having a correlate in the target, that is, that have been mapped one by one onto some relationships in the target. Relations pertaining to $\mathrm{R}_{2}$ are those that have been interpreted as mere artifacts of the model, with no correlate in the target system. On the other hand, relationships pertaining to $\mathrm{R}_{3}$ are those about which there is no interpretation yet, given the incompleteness of our knowledge about the physical domain. The main point is that, in the partial structures account, mathematics represents a given phenomenon in virtue of those features that are mapped onto the phenomenon $\left(R_{1}\right)$. We do not need a full mapping to use a mathematical model (that is, we do not need to solve all our puzzles about $\left.R_{3}\right)$. 


\subsection{Pincock's Mapping Account of Content}

Christopher Pincock has pointed out that structural resemblance can be trivially established if models are taken to be mere mathematical objects (read: purely non-linguistic entities). Structural relations between two systems are very easy to find if the systems have the right cardinality, and so if that is all that it takes for a model to be representative, any model with the right cardinality can represent anything. This is true for total and partial structures (and in fact is even more pressing for partial structures, since you do not need two systems having the same cardinality). This problem was pointed out many years ago by Max Newman to Bertrand Russell's view, in The Analysis of Matter, that we can only know the structural features of the external word (cf. Newman $1928,142)$. Newman's problem is, precisely, that since "[a]ny collection of things can be organized so as to have the structure W, provided there are the right number of them", then if it is true that only structural features can be known, "nothing can be known that is not logically deducible from the mere fact of existence, except... the number of constituting objects" $(1928,142)$. In other words, according to Newman, Russell's view entails that if we know the number of objects in the world, knowledge of everything else can be deduced from it. Newman took this as a reductio of Russell's view (see Demopoulos \& Friedman 1985 for a discussion).

According to Pincock, modeling involves a combination of a mathematical entity and a series of propositions relating parts of the model to physical structures. Structural relations cannot be established without the propositions that explicitly relate parts of the two structures. These propositions are an essential component if we want models to meaningfully represent their targets $(2005,1252)$. Pincock calls this view the 'mapping account of content'. A model is accurate with respect to the relevant aspects of a physical system when its content is correct with respect to these aspects. Pincock holds that "the content of mathematical scientific representations... is exclusively structural" $(2012,25)$, and so for him the correctness of a model is determined by the accuracy of the structural relations between the mathematical entity and the physical system in question; but the crucial point is that, if these structural relations are to be meaningful (i.e. nontrivial), there must be a linguistic specification ${ }^{21}$ of which parts of the model represent physical structures $(2005,1252)$ :

The conditions of correctness that... representations impose on a system can be explained in terms of a formal network of relations that obtain in the system along with a specification of which physical properties are correlated with which parts of the mathematics (Pincock 2012, 25; my emphasis).

For Pincock, a representation is meaningful when it truly captures a real structure in the target. Pincock distinguishes between "true representations" (when there is in fact a real isomorphism between the mathematical model and the target), and "false representations" (when there is no such isomorphism) $(2005,1253)^{22}$. The idea is that although one can 'trivially' establish isomorphisms between the mathematics and the target, one only has a real representation when

\footnotetext{
${ }^{21}$ Note that Pincock's use of this word is different from Weisberg's (see Weisberg 2007b, 217; and section 12.1. above).

${ }^{22}$ I assume that 'true' here means correct, and that 'false' means incorrect.
} 
the required isomorphism "actually exists" $(2005,1253)$, that is, when the physical world has a real structure as described by the mathematical model.

But what is the nature of these physical structures, and how can they be related to mathematical structures? According to Pincock, the way structural relations are established is by a process of instantiation. Following Mauricio Suárez, Pincock argues that once the relevant physical relations have been identified in a given context, the system fixes an associated mathematical structure - and in that sense "the system instantiates that structure, relative to that specification, and allows that structural relations are preserved by this instantiation relation" $(2012,29)$. This process of instantiation may start as an assumption on the part of the theorists: they focus on some aspects of the target, rather than on others, so that a given mathematical structure, rather than another, is instantiated. If it turns out that they have focused on the right aspects, then they have discovered the real structure instantiated by the target, and the model truly represents the target in virtue of there really being an isomorphism between the two. And what is more, for Pincock this physical structure is an instance of a mathematical structure. In other words, for a real isomorphism between the mathematical model and the target to occur, what is important "is the mathematical structure found in the target system itself" (2011, 212; my emphasis). ${ }^{23}$ This is how Pincock avoids Newman's problem: although in principle a physical system can instantiate many mathematical structures, only those that are fruitful to scientific theories exist.

\subsection{Assessment of Pincock's Mapping Account}

Pincock's account has strong ontological implications. For example, since the structure in question is taken to belong to the physical system itself, this account depends on some form of scientific realism; and since this structure is taken to be mathematical, the account also depends some form of mathematical realism. I take it that these are shortcomings of the view. As I will show below and in further chapters, one can provide an account of the applicability of mathematics in science while at the same time remaining neutral with respect to debates on realism.

\section{a) Instantiation}

The notion of instantiation, for example, can be interpreted differently from Pincock's view. Mauricio Suárez, in a paper that Pincock himself quotes, defines representation as a 3-place relation that includes not only a structural relation between mathematics and the world, but the intentions and interests of the scientists and the scientific community (cf. 2010, 96). This may sound like a different wording of Pincock's idea, since, as we have seen, for Pincock verbal specifications (which arguably include the intentions and interests of the scientists using the model) are a requisite for establishing structural relationships between mathematics and the world. But the crucial difference is that for Suárez whether or not a physical system 'instantiates' a given structure is entirely relative to those interests:

\footnotetext{
${ }^{23}$ In section $\S 30$, I come back to this notion of instantiation.
} 
Since there are always different ways of cutting out its domain of elements and relations, every physical object instantiates simultaneously several structures. The physical world underdetermines its mathematical structure - which may only be ascribed under a particular description $(2010,96)$.

The idea is that "every object or system instantiates more than one structure" $(2010,96)$, depending on which aspects of it are the focus of our attention, and in that respect mapping from physics onto mathematics is user-relative. So, when we say that a physical system instantiates a mathematical structure, this must be understood as a notion that depends on the approximations, idealizations and abstractions that the scientist has deemed relevant for representing a given physical system. It is this essentially pragmatic element in modelling that prevents us, according to Suárez, from taking the notion of instantiation in a strong metaphysical sense. On this view, instantiation means that scientists have decided to use a mathematical representation in order to highlight certain relevant physical features. As we will see momentarily, this notion of instantiation and its usefulness in science does not justify beliefs in the physical world having mathematical structures (as defined, for example, by mathematical structuralists such as Shapiro or Resnik.) ${ }^{24}$

This has implications for Pincock's notions of true representations, real isomorphisms, and real structures. It does not make sense, I contend, given the notion of instantiation that both Suárez and Pincock endorse, to say that a system incorrectly instantiates one mathematical structure but correctly instantiates another. As we saw, a structure is defined as the formal network describing the set of relations that obtains between some set of objects. There is nothing in this definition restricting the kind of relations that must obtain. As long as a physical system has an adequate cardinality, it can be taken to instantiate (on this view of instantiation) several mathematical structures, and it can be understood as bearing a relation of (real) isomorphism to a mathematical model based on such mathematical structure. So, either all the mathematical structures instantiated by a physical system are real, or none of them is.

Let me elaborate on this. A mathematical structure is supposed to be instantiated by the target when there are instructions that relate specific parts of the mathematical structure to specific parts of the target, thus defining a concrete structure. A different specification would describe other parts of the physical structure as instantiating a mathematical one, and so on. From this point of view, therefore, there is a plethora of instantiated mathematical structures. This is, however, a process of abstraction (as defined above): we decided to focus on some aspects of physical systems, and the relations between those aspects define a physical structure. We then represent this physical system using mathematics. My problem with this is that I do not see why these physical structures should be called mathematical. It cannot be because they are useful in science, because as a matter of fact most of those supposed mathematical instantiations will be of no use for scientific purposes. Once it is accepted that there may be some mathematical instances in the world, there is nothing that prevents us from assuming that all of these mathematical instances exist, regardless of whether they are useful in science or not, because the notion of instantiation is independent of the notion of being scientifically useful. But if we want to

\footnotetext{
${ }^{24}$ According to Otávio Bueno, this notion of instantiation ends up being so weak that it is confusing to keep using that term to refer to this practice (personal conversation).
} 
restrict our commitments to those structures that are indeed useful in science, arguably they are useful in virtue of the physical properties that the specification highlights; and in that sense, it is useless to call them 'mathematical' structures. Therefore, contrary to Pincock, the notion of isomorphism can be understood without positing mathematical instances in the physical world. But, as Bueno suggests (fn. 24), there are other ways of capturing this idea that avoid using this metaphysically loaded term.

\section{b) Assumed Physical Structures}

Here is another reason why it is not necessary to assume Pincock's view that applied mathematics works by describing the underlying 'mathematical' structures of the physical world. In the application of mathematics, whenever a partial isomorphism (or homomorphism) is established, we are assuming that the physical system has a certain physical structure. The physical world can be carved up in many different ways, depending on the features of interest in a given situation, and once the relevant features have been identified one can define a physical structure, which is then associated with a mathematical structure partially isomorphic (or homomorphic) to it. As Bueno \& French put it:

[M]athematics is applied by bringing structure from a mathematical domain... into a physical, but mathematized domain... What we have, thus, is a structural perspective, which involves the establishment of relations between structures in different domains (Bueno \& French 2012, 88).

Let me say something about this 'mathematization' of the physical domain. Bueno \& French rely here on Patrick Suppes' view that the application of mathematics to the world does not occur in a direct way, but through a series of models and steps, organized hierarchically, that mediate between the theoretical models and the target system. The basic point is that in order for a physical phenomenon to be studied mathematically, it has to be first measured, and the process of measurement implies in itself a mathematization, i.e. the assignment of mixed mathematical/physical statements, which are then represented as data sets. These data sets are at the basis of a whole hierarchy of models which end with theoretical models at the top. ${ }^{25}$ The point is that structural relations hold in virtue of the fact that the assumed physical structure is from the start represented mathematically.

Now, in some cases, this assumed structure is more easily identified than in others. For example, in the case of a street map:

[I]t seems natural enough to divide the world up into streets, rivers, coastlines and the like, but in general this will not be the case. When there is a natural candidate pre-theoretic structure, the mapping account can employ this structure. When there is no such structure, we might impose some suitable structure or other and let the resulting mathematical model help us to fine tune or revise the starting structure. Either way, the

\footnotetext{
${ }^{25}$ The literature on this topic is vast, but the locus classicus are Suppes (1962) and Bogen \& Woodward (1988).
} 
mapping account does require having what we shall call an assumed structure in order to get started. There is no avoiding such an assumption (Bueno \& Colyvan 2011, 347).

This assumed structure is physical; not mathematical. But does this mean that this assumed structure is, so to speak, the ultimate underlying structure of the world? It is true that the choice of features of interest can have an element of arbitrariness; but the epistemological gains of dividing the world in one way rather than another are (arguably) not arbitrary. This, however, does not necessarily entail scientific realism. Explanatory power, for example, has often been taken to be an important tool for deciding whether some ways of dividing the world are more natural than others, and so a scientific realist may use this to argue that the success of the model employing an assumed structure is a reason for believing that such assumption is correct or approximately correct. But a scientific antirealist could reply that we can never corroborate whether that assumption is true, and that the best course would be to remain agnostic.

\section{c) The role of pragmatic considerations}

One final point about Pincock's account is that it does not say much about the role of the pragmatic considerations involved in the application of mathematics. It is true that he mentions that the propositions linking the mathematical and physical structures are an essential component of the model, without which "the model would just be a mathematical entity" (2011a, 27). However, in Pincock's account, the role of these propositions is limited to the establishing of the associated mathematical structure, and once this has been done, "inferential claims follow immediately" $(2012,28)$. In other words, for Pincock the background knowledge of the scientist is important insofar as it fixes the content of the mathematical representation; but once this content is fixed, there is a guarantee that anything discovered in the mathematical model will be a discovery in the target. I believe, however, that this is not necessarily the case. As we will see, scientists must interpret the mathematical formalism at different stages of the application process. So, although it is true that Pincock's account leaves room for "the beliefs and goals of the scientists which deploy the model" (Pincock 2011b, 213), for him these considerations begin and end once the mapping between the physics and mathematics has been fixed. I believe that in some cases, this will be true; but a general account of the application of mathematics should include those cases where additional pragmatic and contextual considerations play a more prominent role.

\section{$\S 14$. The Inferential Conception of the Applicability of Mathematics}

Conceptually, the Inferential Conception of the applicability of mathematics can be understood as building upon, and enhancing, Pincock's mapping account. Both accounts rely on the notion of partial mappings between mathematical and physical structures, and both mention the importance of contextual and pragmatic considerations in the application process. The inferential conception, however, goes beyond Pincock's mapping account in the emphasis on the interpretation of mathematical structures in this process, which opens the possibility of having different kinds of mappings involved in the same situation of applied mathematics. 


\subsection{The importance of interpreting mathematical structures}

Otávio Bueno, just as Pincock does, argues for the importance of interpreting the mathematical structures used in a model. The idea is that the same mathematical structure can be interpreted in many different ways, and an analysis of the mathematics alone cannot determine which interpretation is correct $(2012,980)$ :

[M]athematical expressions, taken by themselves, are not about physical events in the world: they need, first, to be (properly) interpreted before they can become relevant to the description of physical phenomena. A differential equation has multiple interpretations, and depending on the interpretation one adopts, the equation may provide no implications at all to the physical world, or implications that turn out to be empirically inadequate, or implications that, suitably reconstructed, may capture some aspect of the physical world $(2012,973)$.

The idea is that, whether a substructure of the model belongs to $R_{1}, R_{2}$, or $R_{3}$ is not straightforwardly deducible from the model itself. As Bueno puts it, without an interpretation, "mathematics does not state anything about the physical world" $(2012,973)^{26}$. Let me illustrate this point with two examples, the first one is a simple case adapted from Bueno and Colyvan (2011) and the second is taken from Bueno (2012).

Imagine an object dropped from the top of a $20 \mathrm{~m}$ high building. We want to calculate the time it will take for the object to reach the floor:

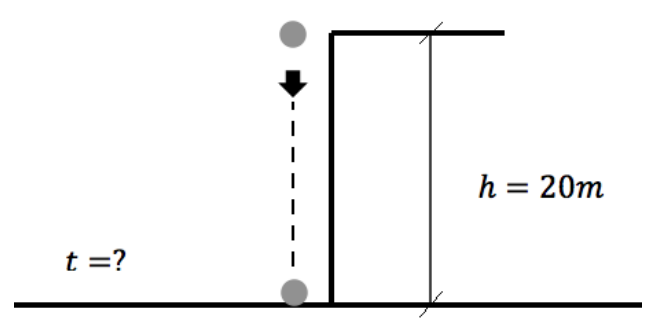

fig 2.

In order to calculate time $t$, we can use the model specified by equation (a), replacing the values for the height $(h=20 \mathrm{~m})$ and the acceleration due to gravity $\left(g=9,8 \mathrm{~m} / \mathrm{s}^{2}\right)$ :

$$
\begin{aligned}
& h=\frac{g t^{2}}{2} \\
& 20=\frac{9,8 t^{2}}{2}
\end{aligned}
$$

\footnotetext{
${ }^{26}$ As we have seen, Pincock also emphasizes this point.
} 


$$
\frac{40}{9,8}=t^{2}
$$

From this, we obtain: $2,02=t_{1}$ and $-2,02=t_{2}$. As we can see, despite the fact that one can establish a mapping between the model specified by (a) and the system (suitably mathematized), there are elements of (a) that are problematic, notably, that (a) has two real solutions for $t$, only one of which is physically significant (in terms of partial structures, we can say that one solution belongs to $R_{1}$ and the other belongs to $R_{2}$ ). Now, what is crucial is that the decision of rejecting the negative solution cannot be drawn from the analysis of equation (a) itself (Bueno \& Colyvan 2011 , 349). On the contrary, that decision is part of the background knowledge of the theorist, who dictates to interpret the mathematics in a specific way.

We can also see this in the case of Paul Dirac's discovery of positrons, cited, among other places, in Bueno (2012). The Dirac equation admits negative solutions for the variable representing electron particles. At first, these solutions were thought to be mere artifacts of the mathematics, with no physical implications (that is, they were assigned to $R_{2}$ ). Later on, Dirac assigned an interpretation. He took them to refer to 'holes' in space-time. This interpretation, however, proved to be inconsistent with the theory - it implied infinitely massive particles! Finally, he provided a third interpretation of the same mathematical structure. This time the solutions were interpreted as referring to particles as massive as electrons but with opposite charge, thus giving rise to the idea of positrons, whose existence was later experimentally confirmed. So far, the positron interpretation of the mathematical formalism has turned out to be the correct one (Bueno 2012, 974). Again, the solutions to the Dirac equation were by themselves meaningless, since many different interpretations could be assigned. It is only when a correct interpretation is advanced that the mathematical formalism captures aspects of the world (see Bueno 2005 and 2012 for details; also Bueno \& Colyvan 2012, 364-65).

These examples illustrate the importance of interpretation in the application of mathematics. As Bueno and Colyvan put it:

[A] II of the interpretations in question are mappings from a mathematical structure to the empirical set up, and they are not uniquely determined by the structure alone - hence, the need for pragmatic and contextual considerations in the selection of suitable mappings (Bueno \& Colyvan 2011, 365).

This does not mean, however, that, because structural resemblance must always be understood in terms of interpreted mathematical structures, there are no constraints in the mathematical model to be used. For example, although it is true that one can represent a city using several different maps, a map is still better than a white page; and, as James Ladyman has pointed out, to deny this seems to take us away from what we take to be a scientific representation (Ladyman 2009, 421). A fruitful mathematical model will be one that allows us to infer many things about the world. At the opposite side of the spectrum, however, models from which 'everything follows' are also not useful (like, for example, models with inconsistent idealizations). As Peter Tan has emphasized, there must be some constraints with respect to the kind of inferences that are admissible (cf. Tan, 'The Challenge of Inconsistent Idealizations' (unpublished draft)). 


\subsection{The basic structure of the Inferential Conception}

The inferential conception integrates both structural resemblance between mathematical models and the world, and "additional pragmatic and context-dependent features" (Bueno \& Colyvan 2011, 352). This account is presented in three papers by Bueno \& Colyvan (2011), Bueno \& French (2011), and Bueno \& French (2012), and more recently in Bueno \& French (2017). According to the inferential conception, the process of application of mathematics in science can be understood in terms of the following three steps:

In the immersion step, a mapping is established between the empirical set up to a convenient mathematical structure. Several mappings can do the job, and so the choice of mapping is a contextual matter (cf. Bueno \& Colyvan 2011, 353).

In the derivation step, one draws consequences from the mathematical formalism, using the mathematical structure obtained in the immersion step (cf. Bueno \& Colyvan 2011, 353).

Finally, in the interpretation step, one interprets the consequences that were obtained in the derivation step in terms of the target. Contrary to Pincock's account, this mapping is not necessarily the one used in the immersion step (cf. Bueno \& Colyvan 2011, 353-4).

The basic idea of the inferential conception is that the representation process involves a mapping between the target and a mathematical model, upon which certain mathematical operations take place, resulting in a mathematical structure that maps onto the target once it has been interpreted in a suitable way. The following scheme represents the essential features of the Inferential Conception:

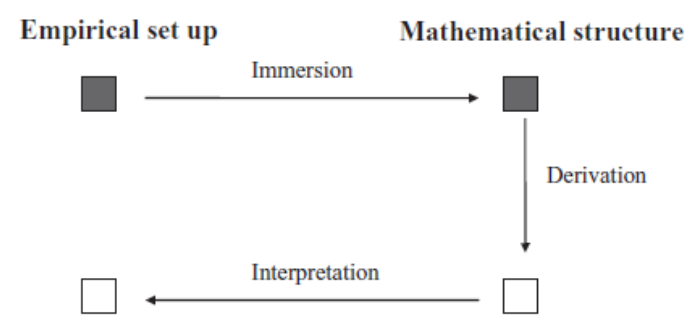

fig. 3

(Bueno \& Colyvan, 2011, 353) $)^{27}$

The inferential conception leaves room for the incompleteness of our knowledge of the physical domain, and so it is also compatible with the partial structures view:

[W]e typically have surplus structure at the mathematical level, so only some structure is brought from mathematics to physics; in particular, those relations which help us to find

\footnotetext{
${ }^{27}$ See also Bueno \& French 2012, 102.
} 
counterparts, at the empirical domain, of relations that hold at the mathematical domain. In this way, by 'transferring structure' from a mathematical domain to a physical domain, empirical problems can be better represented and tackled (Bueno \& French 2012, 88).

Bueno \& French point out that although there may be different structures that may count as surplus, the choice of one structure over the others is decided on a contextual basis $(2017,22$ fn36).

The Inferential Conception explicates the different roles mathematics plays in science, for example, in unifying seemingly disparate phenomena, in making predictions, and in scientific explanation. The main idea of the inferential conception is that these roles can be understood in terms of the main role of drawing inferences within mathematical structures that correspond to physical relations. As Bueno and Colyvan put it:

[B]y embedding certain features of the empirical world into a mathematical structure, it is possible to obtain inferences that would otherwise be extraordinarily hard (if not impossible) to obtain $(2011,352)$.

Thus, in unifying different scientific theories, one establishes inferential relations between them, like showing that one can be derived from the other $(2011,352)$. In the case of novel predictions, one infers from some initial conditions that have been embedded in a mathematical structure, that, given some properly interpreted mathematical results, a given phenomenon may or may not occur (2011, 352). Finally, scientific explanations are formulated in terms of suitable interpretations of inferred mathematical results $(2011,353)$.

As we will see, it is because of this interpretation element that the Inferential Conception is best equipped to deal with some issues concerning the role of mathematics in scientific explanation.

\section{$\S 15$. Mathematics and Scientific Explanation}

\subsection{The Representational Role of Mathematics}

According to the Inferential Conception, explanations are formulated by interpretations of the mathematical formalism in terms of the target. Crucially, what does the explanatory work is not the mathematical formalism per se, but the empirical features represented or highlighted by these mathematical formalisms. On this view, mathematics is a device to find out about these explanatory relationships.

[A] stone is thrown into the air. At one point in time, the mathematical equation that describes the stone's movement has value zero. Does the fact that the equation has such a value provide an explanation of why the stone is at rest, or does it simply offer a mathematical description of the phenomenon in question? Presumably, no one would consider the fact that an equation has value zero to be by itself an explanation of a physical phenomenon. A suitable physical interpretation, which identifies the relevant physical 
processes responsible for the production of the phenomena in question, is needed in order to yield a satisfactory explanation (Bueno \& French 2012, 104).

This way of understanding the role of mathematics is compatible with all the accounts of scientific explanation we have studied in Chapter 1 . The main idea is that mathematics helps us identify the physical features that are responsible for the explanation. Hempel's DN model, for example, assigned a representational role to mathematics. In the DN model, deduction is the relevant explanatory tool, mathematics being just a tool to carry out these deductions. As Hempel puts it:

[The function of mathematics] is analytic or explicative: it renders explicit certain assumptions or assertions which are included in the content of the premises of the argument. [...] Mathematical as well as logical reasoning is a conceptual technique of making explicit what is implicitly contained in a set of premises (Hempel 1983, 390).

And then he adds:

Thus, in the establishment of empirical knowledge, mathematics... has, so to speak, the function of a theoretical juice extractor (Hempel 1983, 391).

In the case of Salmon's CM model, only causal processes and interactions are explanatory; since mathematical entities and operations do not have causal powers in Salmon's sense (as processes that transfer a conservative quantity as described by a conservative law ${ }^{28}$, the role of mathematics cannot be that of explaining the explanandum. The mathematics in the explanans is used to represent those causal processes and interactions.

In the case of Woodward's interventionist account, scientific explanation requires information about the invariant relationships of dependence responsible for the occurrence of the explanandum. We saw that an invariant relationship is one that remains stable under interventions, so that it is possible to manipulate one relata by intervening on the other. Sometimes it is impossible to carry out these interventions, and yet we can gather this information by analyzing a mathematical model that represents these relationships, as long as we have, of course, an appropriate model. Thus, for example, we can explain the tides by citing the position of the moon at a certain point in time. Despite a 'surgical' intervention over the moon's position not being possible, we can 'change' its position in the mathematical model that represents it, for example, if we insert in the variable representing its distance from the Earth a value different from the actual one. This allows us to identify distance from the Earth as one of the causes of the tides. Here, the mathematical model is a tool to manipulate these objective relations of dependence in the world.

\footnotetext{
${ }^{28}$ There are other ways of interpreting mathematical objects as having causal powers. James Cargile, for example, holds that for a mathematical Platonist the intuition of mathematical truths can be the cause of some physical processes (like my physical behavior consisting in writing physical marks on a physical paper) (see Cargile 2003, 145 for details). Field (1989) interprets Gödel's mathematical intuition in a similar way.
} 
Now, Woodward limited his analysis to causal relationships, but, as Alisa Bokulich (2011) has suggested, and we will see in section $\S 18$, noncausal relationships can also be included. Bueno \& French (2011) point out, following Alisa Bokulich, that even in noncausal explanations the mathematics should "be able to give information about how the target system would behave, if the structures represented in the model were changed in various ways" (98). One can certainly use a mathematical model as a means of performing hypothetical manipulations of the target system.

\subsection{Can mathematics be explanatory?}

The consensus that the role of mathematics in scientific explanation is merely representational has recently been challenged by some authors who claim that, at least in some cases, mathematics can play an explanatory role, in addition to its representational role. These supposed cases have been called Mathematical Explanations of Physical Phenomena (MEPPs). Robert Batterman (2010) distinguishes between MEPPs that appeal to mathematical entities and those that appeal to mathematical operations. The first kind of cases would show that, despite their non-concrete nature, mathematical entities and their properties can be genuinely explanatory. These cases have recently been the focus of debates centered on the indispensability of mathematics in science as a justification for mathematical realism. This kind of MEPP is the main focus of this dissertation, and I will thoroughly discuss it in chapters 3 and 4, where I develop an account of what is and what is not special about these cases. The other kind of MEPP are those that involve mathematical operations. The discussion of this second kind of MEPP is important for highlighting the scope and limitations of the Inferential conception, which is the main focus of the present chapter. So, let me start discussing one example of a MEPP that involves, in an essential way, a mathematical operation.

\section{a) The Rainbow Case}

According to Batterman, mathematics can play a genuinely explanatory role in science, one that is independent of its representational role. He introduces the notion of 'asymptotic explanations', which are those that indispensably involve mathematical operations. According to him, what is special about these cases is that there is a transition between two theoretical models used to represent the same phenomenon, but where the underlying theories are different, only one of which is currently taken to be true by the scientific community. Model A aims to be an accurate representation of the target, and model $B$ is a more idealized representation of it. Crucially, model $B$ results from model $A$ by asymptotic reasoning, that is, by taking the limit to one relevant variable of model A. For Batterman, what is important in this kind of case is that the mathematical operation (in this case, taking the limit) that relates the two models is essential for the explanation to work as an explanation. Asymptotic explanations, Batterman argues, "do not proceed by focusing on an abstract structure realized by the physical system" (2010, 3), and "do not require that one associate a mathematical entity or its properties with some physical structure had by the system of interest" $(2010,4)$. Rather, they rely on the mathematical operation of taking the limit to a parameter in the model. 
Let us examine one case often cited in the literature:

THE RAINBOW CASE: Rainbows always appear at $42^{\circ}$ relative to the direction of sunlight (that is, the inclination of the light beams coming from the top of the bow with respect to the sunlight is always around $42^{\circ}$ (See fig. 4). In addition, they always appear with the same color pattern, despite the fact that each rainbow is the result of a unique set of circumstances (Batterman 2010, 20). How can we explain these features?

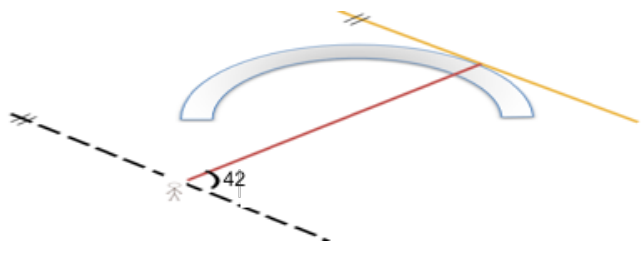

fig. 4

These features of rainbows are due to the interaction between light waves and raindrops, so it is natural to think that we must use light wave theory to explain them. And in fact, using wave theory one can provide an explanation of the color pattern as a consequence of the phenomenon of light dispersion, namely, that the index of refraction varies depending on the wavelength of the refracting waves (the approximate values of the refraction index for colors at the opposite end of the spectrum are: $n_{\text {red }} \approx 4 / 3$ and $n_{\text {violet }} \approx 1,344$.). In turn, this influences the order in which they hit us in the eye, red beams from higher drops all the way down to violet beams from the lower ones, as can be seen in fig 5 :

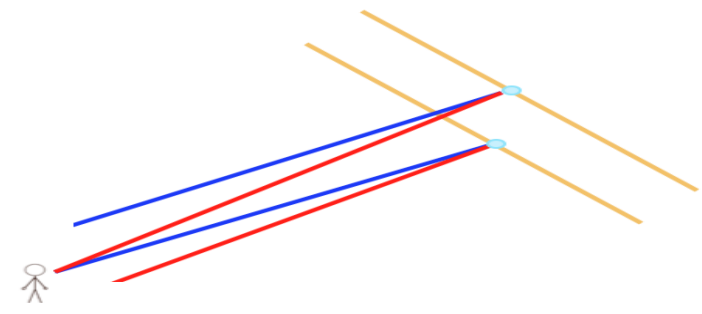

fig. 5

However, in order to explain the inclination itself (why the inclination of diffracted light beams with respect to the direction of sunlight is always approximately $42^{\circ}$ ) we must appeal to the geometrical relations between incident and refracting beams inside each raindrop (assuming spherical raindrops). In the case of red beams, the geometrical relationships are as follows: 


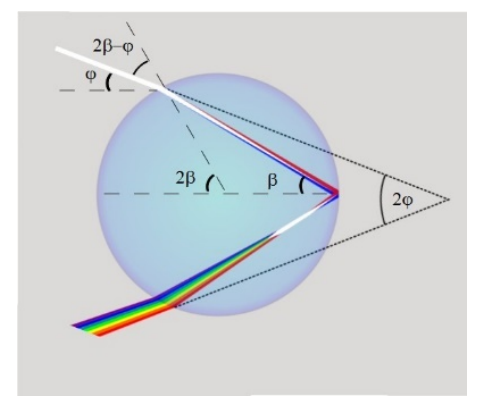

fig. 6

Following Snell's law:

$$
\sin (2 \beta-\varphi)=n \sin \beta \ldots(a)
$$

The largest concentration of rays occurs when the variation of $\varphi$ with respect to $\beta$ is zero; which can be represented mathematically as:

$$
\frac{d \varphi}{d \beta}=0 \ldots(\mathrm{b})
$$

For red light, $n_{\text {red }}=4 / 3$, so we have

$$
\begin{aligned}
& \operatorname{Sin}(2 \beta-\varphi)=\frac{4}{3} \operatorname{Sin} \beta \\
& \varphi=2 \beta-\operatorname{Sin}^{-1}\left(\frac{4}{3} \operatorname{Sin} \beta\right) \\
& \frac{d}{d \beta}[\varphi]=\frac{d}{d \beta}\left[2 \beta-\operatorname{Sin}^{-1}\left(\frac{4}{3} \operatorname{Sin} \beta\right)\right]
\end{aligned}
$$

Using (b) we have

$$
0=2-\frac{\frac{4}{3} \cos \beta}{\sqrt{1-\frac{16}{9} \sin \beta}}
$$

Since

We have that

$$
\cos ^{2} \beta=1-\sin ^{2} \beta
$$

$$
\sin \beta=\sqrt{\frac{5}{12}}
$$

Therefore,

$$
\beta \approx 40,2^{\circ}
$$

And following (a) we have

$$
2 \varphi \approx 42^{\circ}
$$

An analogous reasoning gives us $2 \varphi \approx 40,5^{\circ}$ for violet light. 
This is puzzling. This explanation depends upon the geometrical relations occurring inside the drop (fig. 6), which are captured when light beams are represented as perfectly linear, that is, when light is taken to be traveling as rays. This means that we must not go into the details of the wave composition of light. Rather, we must amplify the scale at which we represent diffracted red light beams. In other words, we must assume red light beams are rays, rather than waves, which is false. The crucial problem, says Batterman, is that this falsehood is essential for explaining the $42^{\circ}$ inclination.

Here is the puzzle in other words: in order to explain the color pattern, we must appeal to properties of diffracted light beams taken as waves (depending on the wavelength, the refraction index will change); but for explaining the inclination itself we must appeal to geometrical relationships occurring inside the drop, which in turn requires describing light beams as rays. Why is a false representation of light essential to explaining a feature of real rainbows? Moreover, why do we need two different models (which assume different ontologies) to explain related features of the same phenomenon?

\section{b) Limiting Operations}

According to Batterman, the reason why this explanation succeeds is that the wave model and the ray model are related by a limiting operation. In the wave model, each diffracted wave has an associated wave length $\lambda$. When the ratio between this wavelength $\lambda$ and the radius $r$ of the raindrop approaches zero, the realistic mathematical representation collapses into the false ray representation. This operation is called taking the limit (also known as variable reduction). Taking the limit, says Batterman, is explanatorily essential because it provides "the mediating link between the representative models" $(2010,10)$. A simple illustration of this is given by a function $f(x)$ that defines a curve in a Cartesian plane.

$$
\begin{gathered}
f(x)=A \cos \frac{2 \pi}{\lambda} x \quad \ldots \text { (c) } \\
\lim _{1 / \lambda \rightarrow \infty} f(x)=\lim _{1 / \lambda \rightarrow \infty} A \cos \frac{2 \pi}{\lambda} x \\
\lim _{1 / \lambda \rightarrow \infty} f(x)=A \quad \ldots \text { (d) }
\end{gathered}
$$

As we can see, equation (c) specifies a wave and equation (d) specifies a line. The limiting operation provides a transition between these two models, and because of that, says Batterman, the highly idealized model of ray optics can be successfully used in the explanation of why the inclination is always the same. The model can be obtained as a limiting case of the more realistic model of wave optics as the result of a mathematical operation performed over the latter (the limiting operation), and in that sense, the operation itself is explanatorily relevant. Batterman's account of asymptotic explanation relies on mathematics identifying stable patterns that omit many details of the actual phenomena. By taking the limit in the mathematical model, one discards these details of the real processes. The goal of the explanation is to show why these regularities occur (as opposed to relying on the regularities to explain something else). 
According to Batterman, asymptotic explanations are a counterexample to mapping accounts, because these explanations rely on the idealized components of the mathematical models, and because the operation itself does not represent anything in the real world. So, these explanations, according to Batterman, do not rely on structural resemblances between the model and the target system, which is the core assumption of mapping accounts. He summarizes this problem as follows:

The problem is simple. Nothing in the physical world actually corresponds to the idealization. So, in what sense can we have a mapping from a mathematical structure to an existing physical structure? Mapping accounts are representative and good representations reflect the truth about the world. Idealizations, however, are false (2010, 10).

Therefore, Batterman argues, a completely new approach to the problem of mathematical application and scientific explanation is needed.

\section{c) Response to the Rainbow Case}

According to Bueno \& French (2012), asymptotic explanations can be incorporated to the inferential conception. It is true that, prima facie, there are no mappings for the limiting operations, and this may be a problem for the inferential account because "if there are no physical analogs corresponding to the divergences and singularities in the mathematical setting, the inferential conception cannot land back in the empirical set up" (Bueno and French 2012, 91). However, Bueno \& French argue that as long as there is some model that can be interpreted in terms of the target system, there is no problem if a model is mapped onto a more idealized model:

A given physical structure can be related via partial homomorphisms (or some other partial morphism) to a suitable mathematical structure, which in turn is related to further mathematical structure, some of which can then in turn be interpreted physically (Bueno \& French 2012, 91).

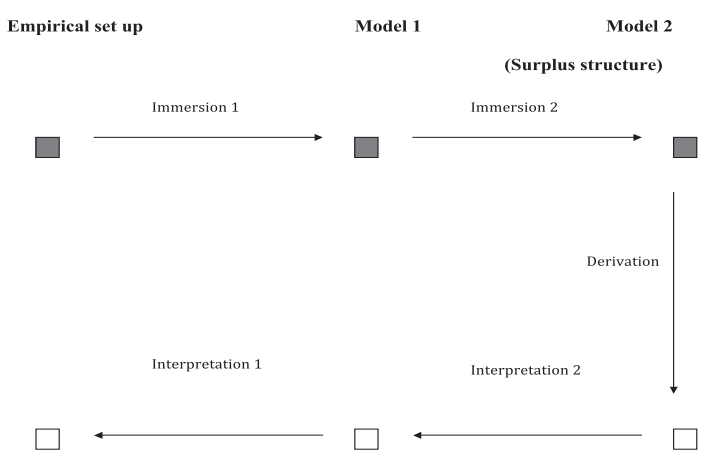

fig 7.

Bueno \& French 2012, 92 
The idea is that the initial mathematical model is itself immersed into another model, which gives us the surplus structure, and the results are then interpreted back into Model 1, which only then is interpreted into the physical set up:

Even if there is no possible physical structure analogous to the surplus structure (where asymptotic reasoning takes place), it is perfectly possible for the intermediary structures... to have a suitable physical interpretation. These intermediary structures ultimately link the surplus structure to the empirical set up (Bueno \& French 2012, 92).

This is called a 'two-stage' mapping. The point is that, although it is true that there is no physical interpretation for the limiting operations, as long as the outcomes of these operations can be interpreted in terms of the empirical set up, the mathematical operation itself does not need to be assigned an interpretation, and remains at the level of surplus structure (Bueno \& French 2012, 92).

This can be seen in the rainbow case. The operation of taking the limit $\lambda / r \rightarrow 0$ connects the two models, but that only shows that the ray model is a special case of the wave model, and so that it can be incorporated under the overarching wave theory of light as a special case, where, once the parameter $\lambda / r$ falls below a certain threshold, some features of wave are irrelevant for explaining the angle of incidence of the rainbow. Bueno \& French's point is that what is doing the explanatory work in this case is the ray model, and not the operation by which we reached that model. After all, even before the advancement of the wave theory of light we could have considered the ray explanation as fairly complete once the refraction index was empirically calculated. The means by which the ray model was conceived are not relevant for the explanation itself.

In addition, as Pincock (2011b) has pointed out, the ray representation does have a structural resemblance to real light beams: the ray representation highlights the fact that some relations within light beams have some sort of 'linearity' (their trajectory), which is essential for explaining the angle of incidence. Representing light beams as rays, although false in many respects, is true nonetheless with respect to this specific feature of light. So, even though it does not directly represent the target, the idealized model can be understood as indirectly representing some features of the target, and in that sense, there is after all a structural similarity between the ray model and the physical light beams. This is, as we saw above (12.3), one of the reasons why minimal models are so useful in science.

\subsection{Are all operations in the derivation step legitimate?}

I would like to finish this chapter presenting one criticism to the inferential conception. To my mind, one aspect that has not been clarified is whether all the operations in the derivation step are admissible. This account seems to put all the explanatory force in the immersion and interpretation steps. Consider for example the discussion in Bueno \& Colyvan (2011): 
[T]o articulate mathematical explanations ${ }^{29}$ it is crucial to establish inferential relations between mathematical structures and the (suitable interpreted) empirical set up. The key inferential moves emerge here in the immersion and in the interpretation steps (366).

What is lacking here is a discussion of the role of the derivation step in conveying understanding of the physical situation. For example, Bueno \& French emphasize that:

A basic requirement [for an explanation] ... is that we understand how the explanans leads, in some sense, to the results in question obtaining, not simply that they do in fact obtain $(2012,102)$.

But then they add that "such understanding may be provided through the identification of suitable physical interpretations of the relevant mathematical results" (2012, 102; my emphasis). If the understanding is provided solely in virtue of considering the mathematical results, then in principle this account seems to be open to the possibility that an explanation can be good even if the derivation step (the means by which those results were obtained) cannot be assigned a physical interpretation. This does not seem right. As we have seen in chapter 1 , a scientific explanation must convey understanding about how the explanandum was brought about. Woodward (2003), for example, says that "not every logical or mathematical transformation one can perform on a formal representation of a system corresponds to a physical manipulation performed on the formal system itself" (196). So, something must be said about the kind of derivations that are admissible.

Moreover, if explanation was merely a matter of properly interpreting the mathematical results obtained in the derivation step, then operations that do not convey understanding would be explanatory, which is incorrect. Here are three such cases:

Brute computation strategies. Consider the proof by exhaustion in the famous bridges of Königsberg case: a bridges system about which it is asked why no one has been able to perform a continuous walk over all the bridges, without retracing one's steps (what is called an Eulerian path). Walking over all possible routes would establish that an Eulerian path over the bridges is impossible, but, crucially, it would not explain why it is impossible (I thoroughly discuss this case in $\S 18, \S 22$ and $\S 27)$.

Mistaken operations. In principle, the inferential conception (at least in the version advanced in the papers I have been discussing) is open to mistaken operations (operations that include errors in calculations or in derivations) as being explanatory in virtue of the results having a proper physical interpretation.

Inconsistent idealizations. The trivial mathematical derivations obtained from inconsistent idealizations that I mentioned in 14.1 above also should be excluded as explanatory (see Tan (unpublished draft) for a throughout discussion; see also Woodward 2003, 58).

\footnotetext{
${ }^{29}$ I assume they mean 'explanations that include mathematics'.
} 
The point of my objection is that something more should be said as to the kind of operations that are admissible in the derivation step.

I believe that the resources for overcoming this difficulty can be found within the Inferential Conception itself. For example, Bueno \& French say that "[a] suitable physical interpretation, which identifies the relevant physical processes responsible for the production of the phenomena in question is needed in order to yield a satisfactory explanation" $(2012,104)$. I think this is correct, as long as we understand 'physical processes' in a broader sense (not in the restricted sense advocated by Salmon's CM model). If the physical relations are emphasized as vehicles for understanding, they can be tracked down by the relevant mathematical derivations, and only those that can be interpreted that way would count as explanatory derivations.

Now, to be fair, in their recent book, Bueno \& French say that the inferential conception does not provide an account of explanation per se. Rather, it is "a framework in terms of which certain kinds of explanations can be articulated" $(2017,201)$. However, they do acknowledge the strength of my objection. They say:

[A]s Barrantes suggests, we might still insist that not all moves in that step are permissible insofar as they may be deemed to fail in supporting the explanation as a whole. Thus, for example, trivial mathematical derivations from inconsistent premises should be excluded (assuming the adoption of classical logic; these inferences are immediately blocked in a paraconsistent setting) $(2017,156$, fn. 208)

Also in response to these comments they add that:

[C]ertain kinds of permissible moves on the mathematical side are going to be ruled out when it comes to the explanation of physical phenomena. Thus, referring back to the diagram of our iterated inferential conception above, when it comes to the 'derivation' step, certain kinds of moves here should be excluded. So, for example, in addition to those moves that do not track the relevant explanatory asymmetries, derivations from inconsistent premises which would be trivial within classical logic would also be ruled out. Likewise, 'brute computation strategies' might also be deemed to be unacceptable (2017, 201).

In sections $\S 18, \S 20$, and $\S 25$ I go deeper into the issue of whether there are some mathematical operations in the derivation step that may not be legitimate in a scientific explanation, but it is pertinent to introduce the main idea here. In the philosophy of mathematics, there is a growing consensus that there is a distinction between explanatory and non-explanatory mathematical proofs. The first convey understanding of the mathematical results in question, whereas the second merely show that the mathematical results obtain. I argue that in some scientific explanations physical systems can be mapped onto a mathematical model in a way such that the question about the physical explanandum can be perfectly represented as a question about a mathematical explanandum, which requires an explanatory derivation. In those cases, I will argue, a non-explanatory derivation would not be legitimate as the only operation in the derivation step, because that operation would not convey understanding, despite the fact that the 
outcome of such derivation can be interpreted in physical terms. And even if one does not fully solve the issue of which mathematical proofs are and which are not explanatory, my point is that the mathematical derivations must provide us some understanding of why the physical phenomenon in question did occur, and not only showing us that it occurred.

\section{$\S 16$. Conclusion}

As I mentioned, there have been several attempts to provide an account of cases where mathematics plays a genuine explanatory role, besides its representational one. Several authors claim that, at least sometimes, mathematical entities (as opposed to Batterman's operations) can be explanatory, in addition to being representational. Although they reject that there are such cases, Bueno \& French give some guidelines as to what it would mean for mathematics to play a genuinely explanatory role:

$[T]$ hose who hold that mathematics does play such an explanatory role owe us an account of the nature of explanation involved in the relevant examples for scientific practice. Expressing it as neutrally as possible, any such account must be able to tell us how the mathematics and the relevant physical phenomena are related in a manner that goes beyond the representation of this relation via deduction or other formal devices. One option would be for such an account to say how it is that the relevant physical phenomenon is brought about. One doesn't always have to appeal to causal factors in explicating this bringing about - one might draw on certain structural [i.e. structural but non-causal] features, for example $(2012,97)$.

In the next two chapters I present an account of mathematical explanations of physical phenomena that is compatible with the inferential conception. Although I will not show that the mathematics go "beyond the representation" of physical relations, I will show that these explanations do not work exactly as ordinary scientific explanations do. 


\section{CHAPTER 3. An Account of Mathematical Explanations of Physical Phenomena}

\section{§17. Introduction}

In this chapter, I introduce my account of Mathematical Explanations of Physical Phenomena (MEPPs). In my view, MEPPs are counterfactual explanations. And despite the fact that MEPPs are explanations of empirical phenomena, they are noncausal.

I start by presenting and analyzing two cases that have been much debated in the literature: the impossibility of performing a trip over all the bridges of Königsberg without retracing one's steps (§18), whose explanation appeals to a property of non-Eulerian graphs (Pincock 2007); and the prime numbered life-cycle of some species of periodical cicadas (§19), whose explanation appeals to a property of prime numbers (Baker 2005). I next introduce my account of MEPPs in terms of the notions of optimal representation (as opposed to the improvable representations that operate in most applications of mathematics); deformations (as opposed to the causal interventions of Woodward's account); and explanatory mathematical derivations (as opposed to Mark Steiner's notion of explanatory proof) (\$20), and revisit my cases to illustrate my view (\$21). I finish the chapter (\$22) by discussing the sense in which MEPPs are different from ordinary scientific explanations, and how do MEPPs account for the explanatory directionality.

\section{§18. Case study 1. The Bridges of Königsberg}

\subsection{The problem}

In the $18^{\text {th }}$ century, the islands of the German city of Königsberg (now the Russian city of Kaliningrad) were connected between themselves and to the land by seven bridges.

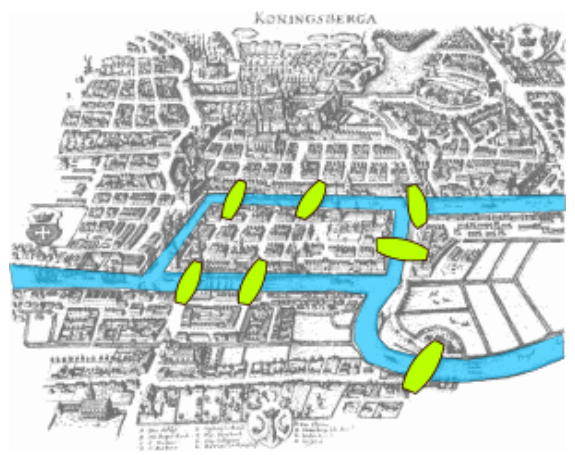

fig. 8. The bridges of Königsberg System

The inhabitants of the city were puzzled about the fact that nobody could do a trip that included every bridge only once. Leonard Euler himself was contacted to solve this problem by Carl Elher, the major of the nearby city of Dunzig. In his letter to Euler, Elher suggested that the solution to this problem may not require traditional geometrical calculus but what Gottfried Leibniz had called 'calculus of position': 
You would render to me... a most valuable service... if you would send us the solution, which you know well, to the problem of the seven Königsberg bridges, together with a proof. It would prove to be an outstanding example of the calculus of position [Calculi Situs], worthy of your great genius (Cited in Hopkins \& Wilson 2004, 201).

In a letter addressed to the mathematician Giovanni Marinoni, Euler wrote about the details of this problem:

A problem was posed to me about an island in the city of Königsberg, surrounded by a river spanned by seven bridges, and I was asked whether someone could traverse the separate bridges in a connected walk in such a way that each bridge is crossed only once. I was informed that hitherto no-one had demonstrated the possibility of doing this, or shown that it is impossible. This question is so banal, but seemed to me worthy of attention in that geometry, nor algebra, nor even the art of counting was sufficient to solve it. In view of this, it occurred to me to wonder whether it belonged to the geometry of position [Geometriam Situs], which Leibniz had once so much longed for. And so, after some deliberation, I obtained a simple, yet completely established, rule with whose help one can immediately decide for all examples of this kind, with any number of bridges in any arrangement, whether such a round trip is possible, or not . . . (Cited in Hopkins \& Wilson 2004, 201).

There are four things to note on this paragraph. First, the way the problem is defined. At the beginning Euler describes it as being about "whether someone could traverse the separate bridges in a connected walk in such a way that each bridge is crossed only once". Presented in these terms, the problem corresponds to the modern graph theoretical one of whether an Eulerian path can be performed over the system of bridges. However, by the end of the paragraph he says that the required path is a "round trip". A roundtrip that crosses all the bridges only once is called, in modern graph theory, an Eulerian circuit. In his essay Solutio Problematis Ad Geometriam Situs Pertinentis (1736; from now on Solutio), however, Euler describes the problem as being about an Eulerian path:

Concerning these bridges, it was asked whether anyone could arrange a route in such a way that he would cross each bridge once and only once (Solutio, Paragraph 2).

The solution he provides in his Solutio applies to Eulerian paths only; if we were to apply it to Eulerian circuits a small modification would be required. Since such a modification was not present in Euler's essay, in what follows I will consider that the problem to be solved is whether an Eulerian path over the bridges is possible. As we will see, only Eulerian and Semi-Eulerian systems allow Eulerian paths.

The second thing to note from Euler's letter to Marinoni is that the question is whether any Eulerian path is possible, and not why a particular attempt failed. Evidently, a negative answer to the first question would apply to the second question as well; but this would not work in the other way around. That is, from a particular failure we cannot conclude that such a path is 
impossible. But if we show that such a trip is impossible we would also be accounting for every particular failure.

A third thing to note is that Euler discards algebra and 'the art of counting' as sufficient to solve the problem. For him, the solution relies on the geometry of position, which around fifty years before had been described by Leibniz as:

[The] kind of analysis, geometric or linear, which deals directly with position as algebra deals with magnitudes (Cited in Hopkins \& Wilson 2004, 201).

This is a kind of analysis in which magnitudes such as distances, lengths or angles are irrelevant. It applies to the bridges case because the real distances between the pieces of land, as well as their areas and the length of the bridges, are irrelevant for solving the problem; the solution requires abstracting away from all these features, considering only the connections between the pieces of land. For this reason, in his solution to the problem Euler did not work directly with the physical system of bridges but on a model that represented the connections only.

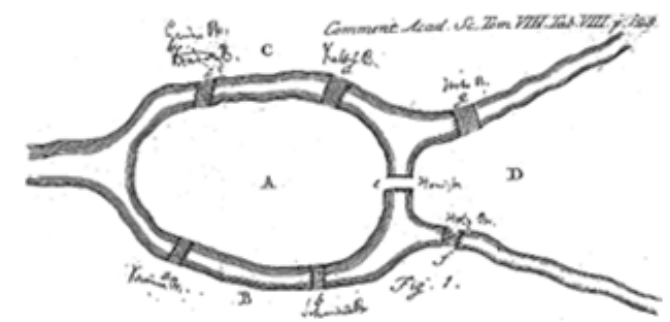

The bridges of Königsberg graph (Euler's version)

fig 9. Baker 2009b, 4

It is often asserted that this was the first instance of a graph theoretical analysis (see e.g. Baker 2009b). In modern graph theory, a graph is a geometrical structure composed of vertices connected by edges. The graph that represents Euler's diagram is the following:

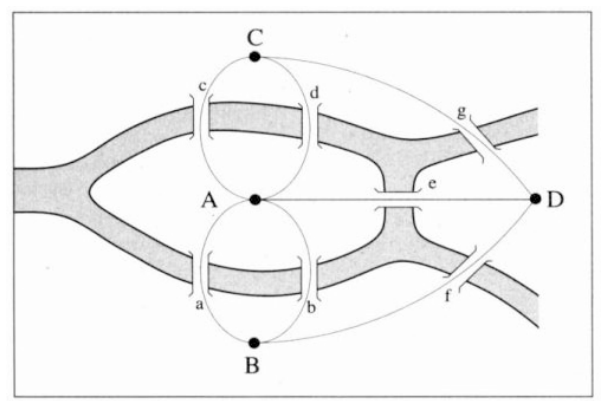

The bridges of Königsberg graph (modern graph theoretical version)

fig. 10. Baker 2009b, 6 
Finally, the fourth thing to notice in the aforementioned quote is that Euler aimed for a solution that did not apply to the Bridges of Königsberg alone; rather, he wanted a rule that would apply to "all examples of this kind, with any number of bridges in any arrangement" (call this 'condition of generality'). As we will see below, abstraction from those elements that are not essential to the solution of the problem is not only desirable for tractability purposes but it is crucial for meeting this condition of generality.

\subsection{Euler's Solutions}

In Solutio Euler presents three solutions to this problem ${ }^{30}$. The first two apply specifically to this case; the third solution is more general and applies to similar cases.

\section{a) Brute computation strategy}

One can systematically perform every single possible trip over the bridges. In doing so, one will notice that none of them is an Eulerian path. Since there is none available, we can conclude that it is impossible to perform an Eulerian path over the bridges system. Euler himself explicitly dismissed this disjunctive explanation, precisely because it does not meet the condition of generality:

As far as the problem of the seven bridges is concerned, it can be solved by making an exhaustive list of all possible routes, and then finding whether or not any route satisfies the conditions of the problem. Because of the number of possibilities, this method of solution would be too difficult and laborious, and in other problems with more bridges it would be impossible (Solutio, paragraph 3).

This is a very important point. The number of possible trips varies exponentially with the number of bridges. A rough estimate for this case is $2^{7}$, assuming that every time one reaches a piece of land, one has 2 options for continuing the trip (cf. Moore and Mertens 2011, 4). But this brute strategy is impossible in cases with many more bridges. In Venice, for example, there are 420 bridges, and even the fastest computer available today would take more than the age of the universe to survey all those options (cf. Moore and Mertens 2011, 4).

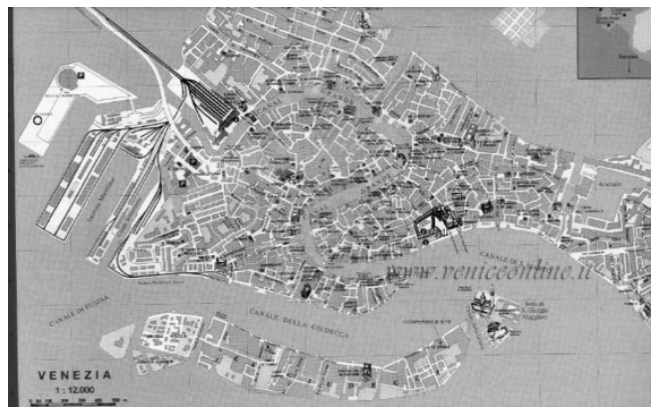

fig. 11. The bridges of Venice

\footnotetext{
${ }^{30}$ A similar classification has been recently highlighted by Tim Räz (2014)
} 


\section{b) Particular analytic solution}

This solution is brilliantly simple. It depends on the extremely simple fact that 'every time one crosses a bridge, two pieces of land are involved: the one from which one departs, and the one where one arrives' ${ }^{\prime 31}$. The solution is divided in two steps. As we can see in fig. 9, Euler labeled the pieces of land with the letters A, B, C and D. He then defined a path as a sequence of letters. First, every time one crosses a bridge two letters appear in the path (those corresponding to the pieces of land at each side of the bridge). From this it follows that if one crosses a second bridge, a third letter would appear in the path-sequence, and so on. In general: "however many bridges the traveler crosses, his journey is denoted by a number of letters one greater than the number of bridges" (Solutio, paragraph 5). If a system has ' $n$ ' bridges, a successful Eulerian path must have ' $n+1$ ' letters. Therefore, in the case of the seven bridges of Königsberg, a successful path would feature 8 letters. And so the question now is whether an 8-lettered path-sequence is possible over the bridges.

Secondly, we must analyze how many times a particular letter will appear in a path, given the number of edges connecting to it. For any piece of land with $K$ bridges connecting to it, either $K$ is odd or $K$ is even (we call it respectively an 'odd piece of land' or an 'even piece of land). It follows, again from the fact that every time one crosses a bridge two letters appear in the path, that if $K$ is odd the letter corresponding to that piece of land will appear $\frac{K+1}{2}$ times, independently of whether or not we start on that piece of land. For example, from fig. 12 it is easy to see that whether or not one starts in L, if one crosses each bridge only once one would step on L exactly 2 times:

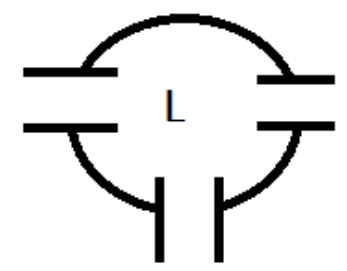

fig. 12

Land $\mathrm{L}$ connected by three bridges

In the bridges of Königsberg, we can see in fig. 9 that there are 5 bridges connecting to A, and 3 connecting to $B, C$ and $D$ respectively, so the frequency of each letter will be:

\footnotetext{
31 I emphasize how simple this fact is because later this will be useful in my discussion of the ontological implications (or lack thereof) of the existence of MEPPs. A bridge is defined as something that connects two areas. It is an analytical truth that every time one crosses a bridge, two areas are involved. This is true for every physical object that meets this description.
} 


\begin{tabular}{|l|l|l|}
\hline & \#bridges & Frequency \\
\hline A & 5 & 3 \\
\hline B & 3 & 2 \\
\hline C & 3 & 2 \\
\hline D & 3 & 2 \\
\hline Total & & 9 \\
\hline
\end{tabular}

Table 1.

In consequence, the number of letters in a path that crosses all the bridges only once must be 9 . But we saw that a successful path should have 8 letters; therefore, the trip is impossible in the bridges of Königsberg system. Although this solution does not meet the condition of generality either, it provided Euler with the elements for constructing a more general solution. Contrary to the brute computation strategy, this solution explains why an Eulerian path over the bridges is impossible. However, the third solution provides a better explanation.

\section{c) Generalized analytic solution}

A generalization of this result should account for cases that also contain 'even' pieces of land. As it is evident from fig 13, in those cases, if the journey starts in an even piece of land its letter will appear $\frac{K}{2}+1$ times in the path sequence. If the journey does not start in that piece of land, its letter will appear $\frac{K}{2}$ times in the path sequence.

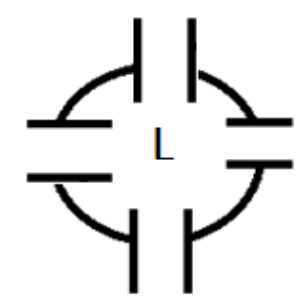

Fig. 13

Land L connected by four bridges

Here is a reconstruction of Euler's generalized solution in modern graph theoretical terms. Define a generalized graph with $o$ odd nodes, $e$ even nodes, and $n$ edges. Every odd node has $h_{i}$ edges connecting to it, and every even node has $k_{j}$ edges connecting to it. A path-sequence is a list of all the nodes covered by a given path. 
(1) Every time you cross an edge, two nodes feature in the path-sequence, the starting point and the ending point [Elementary fact about edges ${ }^{32}$ ]

(2) If a graph has $n$ edges, a successful Eulerian path-sequence will have $n+1$ nodes.

(3) Every odd node will feature $\frac{h_{i}+1}{2}$ times in a successful Eulerian path-sequence.

(4) If the path starts in an even node, it will feature $\frac{k_{j}}{2}+1$ times in a successful Eulerian pathsequence.

(5) If the path does not start in that even node, it will feature $\frac{k_{j}}{2}$ times in a successful Eulerian pathsequence.

(6) Define $r$ as a binary variable that takes value 1 if the path starts in an even node and 0 if it does not.

(7) The number of nodes in any path-sequence is:

$$
\text { \#Nodes }=\sum_{i=1}^{o} \frac{h_{i}+1}{2}+\sum_{j=1}^{e} \frac{k_{j}}{2}+r
$$

(8) In a successful Eulerian path sequence the following holds:

$$
\sum_{i=1}^{o} \frac{h_{i}+1}{2}+\sum_{j=1}^{e} \frac{k_{j}}{2}+r=n+1
$$

$$
\sum_{i=1}^{o} h_{i}+\sum_{j=1}^{e} k_{j}+o+2 r=2 n+2
$$

(10) Every edge has been counted twice, so: $\sum_{i=1}^{o} h_{i}+\sum_{j=1}^{e} k_{j}=2 \mathrm{n}$

$$
\begin{aligned}
& 2 \mathrm{n}+o+2 r=2 \mathrm{n}+2 \\
& o+2 r=2 \\
& o_{1}=2 ; r_{1}=0 \vee o_{2}=0 ; r_{2}=1
\end{aligned}
$$

From this it follows that the desired path is possible under two conditions only:

C1*: Either the graph has exactly two vertices of odd degree (in which case the path must start on one of them) [Semi Eulerian graph]

C2*: Or the graph does not have odd vertices. [Eulerian graph]

\footnotetext{
${ }^{32}$ This fact about edges fully captures the elementary fact about bridges I mentioned above. By substituting edges, nodes and graphs for bridges, pieces of land, and physical road systems this explanation can be cashed out in empirical terms.
} 
These conditions are not met by the graph that represents the bridges system (the graph has 4 odd vertices). Therefore, it is impossible to go over all the edges without crossing over at least one of them more than once.

Euler concludes by saying that this method makes it easier, "even in most complicated cases, to determine whether or not a journey can be made crossing each bridge once and once only" (Solutio, paragraph 16). As I mentioned, tractability is important because brute computational strategies are physically impossible to carry out when the number of possible routes is exponentially large. Even the fastest computer would take more than the age of the universe to process the $2^{420}$ routes available in the bridges of Venice case. Because of this, Moore and Mertens (2011) eloquently describe the importance of this solution in terms of its computational tractability:

An exhaustive verification in a city with $\mathrm{n}$ bridges takes an amount of time that grows exponentially with $\mathrm{n}$. But we can check that every vertex has even degree in an amount of time proportional to the number of vertices, assuming that we are given the map of the city in a convenient format. Thus Euler's method let us solve this problem in linear time, rather than the exponential time of a brute-force search. Now the cities of Venice, and larger cities, are easily within our reach $(2011,4)$.

\section{$\S 19$. Case study 2. The Cicadas}

\subsection{The problem}

Periodical cicadas of the genus magicicada remain underground, in nymphal state, for either 13 or 17 years, getting nutrients from the roots of forest trees. After that period of time they emerge in the summer (when the ground temperature reaches $64^{\circ} \mathrm{F}$ ), mate within the next two weeks, lay eggs, and die. Then the cycle repeats all over again.

These insects are mostly found in the US Eastern states, where 15 different broods have been identified. Broods with 13-year life cycles are located in the South, and broods with 17-year life cycles in the North. One single brood can contain more than one species, but they do not interbreed. In some areas, for example in Virginia, it is possible that broods of 17-year species and 13 -year species geographically coexist, and so they may appear at the same time every 221 years.

Although the number of species of cicadas is a matter of dispute, most biologists agree that there are seven species (cf. Cooley, magicidada.org). Three of them (septendecim, cassini, and septendecula) have 17-year life cycles and each of them has at least one 13-year life cycle counterpart (thus: tredecim, neotredecim, tredecassini, and tredecula). Differences in morphology, behavior and calling signals are clear between species of the same life cycle. However - and here is where the dispute begins - these differences are not so evident between a species and its counterpart with the alternative life cycle. It is for this reason that some biologists claim that these counterparts may both belong to the same species (the only difference between subspecies would be the life cycle length). This question has not yet been settled. Cooley presents the current state of affairs as follows: 
More information on the nature of the boundary between 13- and 17-year populations and the extent of hybridization between them would help to resolve this question, but for now there is no evidence that the distinctiveness of the life-cycle-forms is decreasing. For this reason and for practical purposes, most writers have adopted the taxonomy that recognizes the life cycle siblings as distinct species (Cooley, magicicada.org)

There are many features of these insects' behavior that are puzzling and require explanation, but the most salient is their life cycle. In particular, the question that has puzzled scientists the most is why these insects emerge simultaneously every 13 or 17 years. This question can be decomposed into four sub questions: (i) Why are their life cycles so long? (ii) Why is there a difference between life cycles? (iii) Why do they emerge simultaneously? And (iv) Why are their life cycles prime numbered? As we will see, many philosophers claim that this last question requires a different kind of explanation than the first three; but before we get into this, let us present the standard scientific answers to the first three questions.

With respect to (i) the long length of the cycles, two factors may be involved. Jin Yoshimura (1997) suggests that the colder conditions during the glacial period in the Pleistocene (roughly until 11700 years ago) slowed the growth and development of the cicadas. On the other hand, Cox \& Carlton (2003) suggest that cicadas may have evolved long life cycles so that they can minimize the times they emerge, in order to avoid the risk of emerging during a particularly cold year. Both explanations also account for (ii) the difference in length (Matson 2013). Northern cicadas have longer cycles than southern ones because the climate there is colder (cf. Matson 2013). As for (iii) the synchronized emergence, Matson has pointed out that it is really surprising, especially if we consider the length of the cycles. It may be explained by two factors. First, synchronized emergence increases mating opportunities, and in that sense it is evolutionarily advantageous. In addition, simultaneous emergence increases the chances of survival from predators. Predators have a limited eating capacity. Even at their fullest, they cannot eat the whole population of prey if the number of prey is too large. By emerging at the same time, the cicadas guarantee that part of the population will survive.

As I said before, the feature of these insects' behavior that has generated most discussion among philosophers is that both southerners and northerners have life cycles that are represented by prime numbers. As Matson points out, many subspecies of cicadas may have emerged from the glacial period with a spectrum of life cycles ranging from 12 to 20 years -Yoshimura (1997) suggests that the possible life cycles may have been in the [14-18] range in the North, and [12-15] in the South. Eventually, those with 13 and 17 years survived. Why have precisely those with prime numbered life cycles survived? ${ }^{33}$

\footnotetext{
${ }^{33}$ There may be a little bit of speculation here. These constrains exclude 11- and 19-year life cycles for no apparent reason. In any case, it is still a surprising fact that those of 13 and 17 survived and the rest (most of them non-prime numbered) did not.
} 
It has been argued that the cycles are not 'prime' themselves; that their supposed 'primeness' depends upon an arbitrarily chosen measurement unit: years. Daly \& Langford express this charge thus:

Prime numbers appear in the description of the life cycle of cicadas because, and only because, we measure that life cycle in years. But we could choose to measure the duration of that cycle differently. Instead of measuring it as [say] 17 years, we could measure it as 68 seasons, for instance, or as 204 months. Since neither 68 nor 204 is a prime number, it seems that the role of primes in the life cycle of cicadas is arbitrary... $(2009,652)$.

I am partially sympathetic to this line of thought. As I will discuss below, prime numbers represent life cycles, but the life cycles are not themselves 'prime'. 'Primeness' is a property of some natural numbers, not of time lengths. In the inferential account that I am following, the role of mathematics is to represent physical facts, but these facts are not themselves mathematical ${ }^{34}$ (this is true even in cases where the mathematical representation seems indispensable to pick up the relevant physical properties). So, it seems natural to assume that there is an element of arbitrariness in the choice of the representational tool. However, there is a strong sense in which the charge of arbitrariness is not justified. Years are a natural unit for measuring organisms' life cycles. The rotations of the Earth around the Sun determine climatological conditions that affect the life cycle of organisms by changing relevant local features such as temperature and amount of daylight (cf. Baker 2009a, 615). As I mentioned, cicadas emerge only in the summer, when the ground is at $64^{\circ} \mathrm{F}$. There is only one summer a year, so years are the relevant choice of units for this case. Daly and Langford's claim that months or seasons could also work is right, but only because in both cases the relevant prime factor is included in those numbers. In other words, what is relevant is the relationship between the numbers representing the life cycle and the time of the year where appropriate climatological conditions appear. For example, if we choose months as our unit, we have that the life cycle of northern magicicadas would be 204 units and the climatological conditions would occur every 12 units. Evolutionary time scales are very long, so even if the cicadas emerged during a different month each cycle, on average, they would have to emerge on a 12-unit basis. The relationship between these two numbers would still be prime: 17. It is precisely this primeness that puzzled scientists and philosophers. As we will see shortly, the explanation consists in showing that prime numbers are coprime with numbers less than their double, which can be translated in terms of prime-numbered cycles minimizing intersection with other cycles (more on this below).

It has also been argued that the cicada's prime numbered life cycles may be a mere coincidence. According to this objection, there is nothing to be explained about the cicadas' behavior. This would be supported by the fact that there are no other known magicicadas that develop prime numbered cycles (except for another species of forest cicadas that emerges - nonsimultaneously - every 7 years (see May 1979, 348)). But despite this objection, it is undeniably puzzling that there are no magicicadas of intermediate life cycles. Why precisely 13 and 17 and

\footnotetext{
${ }^{34}$ Sections $\S 37$ and $\S 38$ are devoted to a discussion of these and related issues.
} 
not any other number? ${ }^{35}$ There is still the question of whether there are seven or three species of magicicadas. If the species are seven, then this number may not be negligible, and it makes it less likely that the cycles are a coincidence because the greater the number of species with a property the less likely that the property is an accident (but still, there are more than 1500 other species, most of them developing cycles of less than 5 years). On the other hand, if there are only three species, then the question remains as to why those species have divided themselves in subspecies with prime life cycles. It is not completely clear to me which outcome would be more favorable.

\subsection{The number theoretical explanations}

Two explanations for these prime numbered cycles have been predominant in the literature. Goles, Schulz and Markus (2001) argue that preys with prime numbered life cycles will avoid encounters with predators more than those with non-prime numbered cycles:

[A] prey with a 12-year cycle will meet - every time it appears - properly synchronized predators appearing every 1, 2, 3, 4, 6 or 12 years, whereas a mutant with a 13-year period has the advantage of being subject to fewer predators $(2001,33)$.

This would explain why, amongst the species with different life cycles that may have emerged from the Pleistocene, only those with 13 and 17 years passed on.

The second explanation, due to Cox \& Carlton (2003), emphasizes the evolutionary benefit of not overlapping with subspecies with different life cycles. Most broods include all species with the same life cycle, and there is no interbreeding between these species. However, when two broods overlap in some regions there may be interbreeding between siblings with different life cycles. For example, septendecula (decula with 17-year cycles), and tredecula (decula with 13-year cycles) belong to different broods, but if their broods coincide these two subspecies will interbreed, giving rise to descendants with life cycles between 13 and 17 . These descendants will not overlap with other nymphs belonging to their progenitors' species. This will make them lose the advantage of synchronized emergence. Having prime life cycles ensures that this kind of event happens only every 221 years.

Both explanations rely on the fact that the chosen life cycles minimize the possibilities of intersection (in one case intersection with predators, in the other case intersection with subspecies of different life cycles). Specifically, as Alan Baker puts it, "[t]he mathematical link between primeness and minimizing the intersection of periods involves the notion of lowest [least] common multiple (LCM)" $(2005,231)$.

Lemma 1: the least common multiple of $\mathrm{m}$ and $\mathrm{n}$ is maximal iff $\mathrm{m}$ and $\mathrm{n}$ are coprime.

\footnotetext{
${ }^{35}$ Current research is exploring the possibility that the relevant explanatory property may be the 4-year difference between the two cycles, and not their 'primeness'. If this turns out to be true, then the cicada case would have to be abandoned as an example of a MEPP, because the 4-year difference would have a causal explanation.
} 
Lemma 2: a number $\mathrm{m}$ is coprime with each number $\mathrm{n}<2 \mathrm{~m} ; \mathrm{n} \neq \mathrm{m}$ iff $\mathrm{m}$ is prime.

It seems that without mentioning these facts about prime numbers neither of those explanations of the length of the cycles would work. And although a complete explanation of the cycle lengths must include empirical information about ecological and biological considerations, the fact that cycles of 13 and 17 years minimize the possibilities of intersection requires a different kind of explanation. Moreover, the explanation shows that many aspects of the actual evolutionary history of the cicadas are irrelevant. In other words, that the cicadas would end up having cycles of 13 and 17 years was modally stronger than the specific details of their evolutionary history. The idea is that once the explanandum has been cashed out in terms of primeness, it becomes 'prime-numbered life cycles minimize intersection with other life cycles', and it can be explained by appealing to the two lemmas above, the proof of which is quite simple:

Lemma 1: $\operatorname{LCM}(m, n)$ is maximal iff $m$ and $n$ are coprime ${ }^{36}$
1) $\operatorname{LCM}(m, n) \leq m \cdot n$
[Def. of LCM]
2) $\operatorname{LCM}(m, n)$ is maximal iff $\operatorname{LCM}(m, n)=m \cdot n$
[from 1]
3) $\operatorname{LCM}(m, n) \cdot \operatorname{GCD}(m, n)=m \cdot n$
[Def. of $G C D]^{37}$
4) If $\operatorname{LCM}(m, n)$ is maximal, then $\operatorname{GCD}(m, n)=1$
[from 2 and 3]
5) $\operatorname{GCD}(m, n)=1$ iff $m$ and $n$ are coprime
[Def. of coprime numbers]
6) If $\operatorname{LCM}(m, n)$ is maximal, then $m$ and $n$ are coprime
[from 4 and 5] (first part of the bi-conditional)
7) If $m$ and $n$ are coprime, then $\operatorname{LCM}(m, n)=m \cdot n$
[from 5 and 3]
8) If $m$ and $n$ are coprime, then $\operatorname{LCM}(m, n)$ is maximal
[from 7 and 2] (second part of the bi-conditional)
9) $\operatorname{LCM}(m, n)$ is maximal iff $m$ and $n$ are coprime
[from 6 and 8]

Lemma 2: If $m$ is prime then it is coprime with every $n ; n<2 m$
1) $\operatorname{GCD}(m, n)=1$ iff $m$ and $n$ are coprime
[Def. of coprime numbers]
2) If $m$ is prime, then $\operatorname{GCD}(m, n)=1$ for all $n<2 m$
[Def. of prime]

\footnotetext{
${ }^{36} \operatorname{LCM}(m, n)$ : Least Common Multiple of $m$ and $n$.

${ }^{37}$ GCD $(m, n)$ : Greatest Common Divisor of $m$ and $n$
} 
3) If $m$ is prime then it is coprime with every $n ; n<2 m \quad$ [from 1 and 2]

The notion of primeness is crucial for these mathematical proofs to work. However, as I will argue in more detail in section $§ 37$, there is a strong sense in which the mathematical notion of primeness is not indispensable for explaining the actual length of the cycles. For now, what is important is that the two available explanations of the cycles strongly rely on number theoretical facts about prime numbers. In fact, biologist Robert MacArthur noticed that this "may be the only application of number theory to biology" (cited in May 1979, 347). The cicada's behavior depends upon many different considerations, like evolutionary aspects, climatological aspects, etc., and so a full explanation of the cicada's life cycle requires taking of all of these elements into account. But the crucial point, the reason why many authors think of this as a special case, is that there is a sense in which the scientific explanation that does not appeal to this number theoretical result would be limited in its explanatory power. However, as I will show in $\$ 21$, the role of mathematics in the cicada explanation is to highlight the relevant empirical facts about time lengths that explain the life cycles.

\section{$\S 20$. An account of MEPP}

In this and the following sections I present a characterization of these and similar cases in terms of what has been called mathematical explanations of physical phenomena (MEPPs). The notions that I use will become transparent throughout my discussion.

Assume that we know that a physical system $\mathrm{P}$ has property $\mathrm{p}^{*}$, and we want to explain why it has that property. A MEPP of this fact has the following conditions:

- $\mathrm{M}$ is a mathematical structure that optimally represents those features of $\mathrm{P}$ relevant to explaining why it has property $\mathrm{p}^{*}$.

- $\mathrm{m}^{*}$ is a mathematical property that represents the physical property $\mathrm{p}^{*}$.

- We can manipulate whether $\mathrm{M}$ has $\mathrm{m} *$ by deforming a property $\mathrm{m}$ of $\mathrm{M}$.

- This procedure can be interpreted in terms of manipulations of whether $P$ has $p^{*}$ by deforming a property $p$ of $P$.

These conditions can be interpreted in terms of the immersion, derivation, and interpretation steps of Bueno, Colyvan, and French's Inferential Conception: 


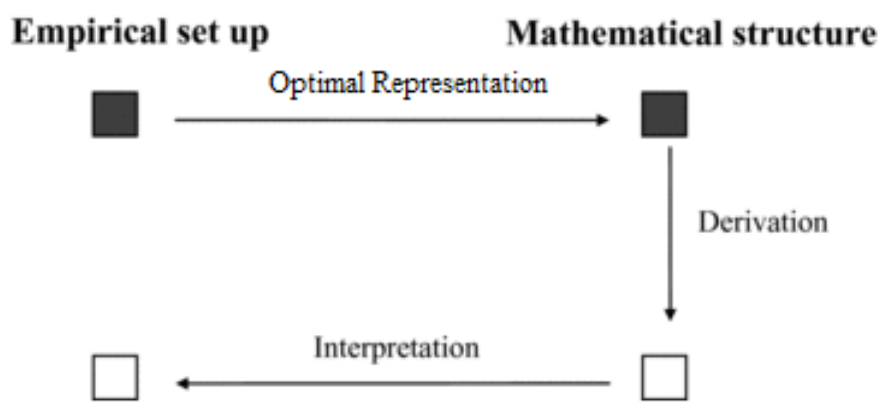

fig. $14^{38}$.

In what follows I discuss the notion of optimal representation, explain the sense in which deformations of mathematical structure $M$ must be understood, and revisit my cases to illustrate my view. I finish the chapter by exploring the relationship between MEPPs and ordinary scientific explanations, and how they account for the explanatory directionality.

\subsection{Optimal Representation}

Mathematical representations can usually be improved. Sometimes this improvement implies new values for the variables in the same mathematical structure, and in others a new mathematical structure must be used altogether. I will call this kind of representation improvable. Consider the following example of a static representation of the relationships between three sticks:

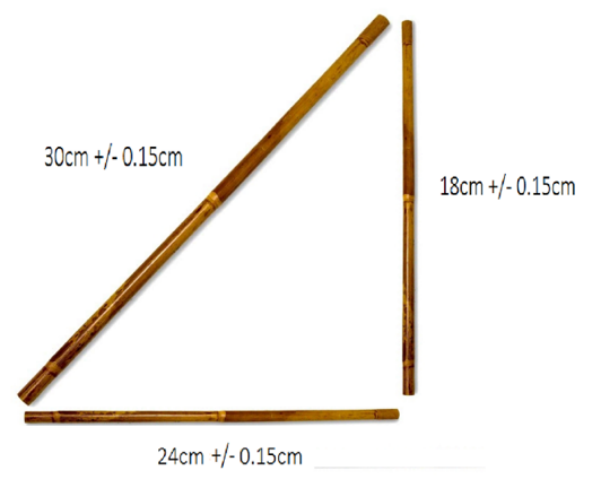

fig. 15

In this case we can use the following mathematical model to represent the geometrical relations between the sticks as a right triangle:

${ }^{38}$ Adapted from Bueno and Colyvan 2011, 353. However, these authors do not use the idea of optimal representation. 
Model 1:

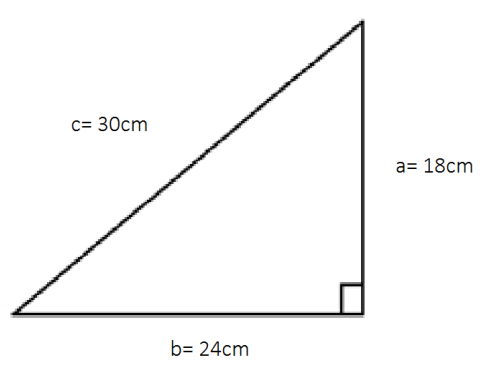

fig. 16

$$
c=\sqrt{a^{2}+b^{2}}
$$

The measures of $a, b$ and $c$ are of course approximate. Depending on the purpose of the representation, we can increase the accuracy of these measures; if we do so, the corresponding mathematical model would no longer be a right triangle:

Model 2:

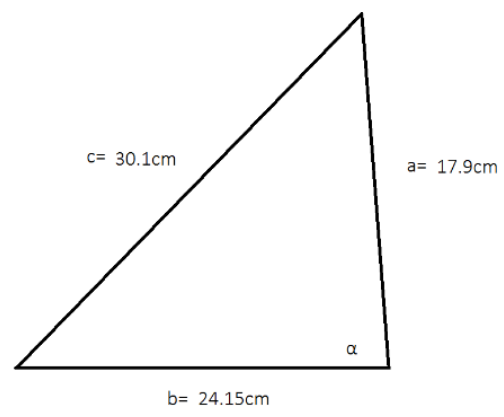

fig. 17

$$
c=\sqrt{a^{2}+b^{2}-2 a b \cos \alpha}
$$

Neither $a, b$, or $c$ are completely straight, but perhaps for making the model more tractable (Galilean model), perhaps because all that matters is the distance between the extremes of the sticks (minimal model), segments have been used in the representation. What is important is that regardless of the representational ideals of the modeler, one can continue improving these representations, either because not all the core features have been identified, or because, having been identified, they cannot be measured accurately. In other words, both Galilean and minimal models are, in general, improvable. For example, a minimal modeler can improve her model by measuring more accurately the distances between the extremes of the sticks, as well as the 
angle $\alpha$. Similarly, a Galilean modeler can provide a better representation if she introduces, for example, aspects of the geometry of the edges of the sticks.

I shall now define a special kind of mathematical representation that I will call 'optimal (i.e. 'non-improvable') representation'. An optimal representation is a minimal model that captures all the features that are relevant to an empirical situation at a specified level of description. ${ }^{39}$ What is a representational ideal for most minimal models is actually achieved by these optimal representations. Let us illustrate this in the hand shaking case of section $\S 12$ :

HAND SHAKING CASE: There are 10 persons in a room shaking hands with each other. How many hand shakings will there be? (see fig. 1)

In order to answer this, we must abstract away from the particularities of the people involved, and the details of every particular hand shaking. The only feature relevant to answering this question is the fact that there will be hand shakings (i.e. the 'connections') between every pair of persons. For that reason, we can use a graph to represent this case. As we have seen, for this particular situation what we need is a complete graph. This graph-theoretical representation is optimal, in the sense that it captures all and only those aspects that are relevant to answering the question about the number of hand-shakings.

Now, mathematical representations are not 'optimal' in themselves. Optimality is a relative notion. A mathematical structure $M$ is an optimal representation of a physical situation $P$ only relative to a specific feature $\mathrm{p}^{*}$ of $\mathrm{P}$. In the case of scientific explanations, $\mathrm{M}$ may be an optimal representation of $\mathrm{P}$ with respect to explaining why it has property $\mathrm{P}^{*}$, but it may not be optimal with respect to explaining another feature $q^{*}$ of $P$. It is also important to note that the mathematical model does not tell us what is relevant in these cases. It is with our background knowledge about the overall situation that we construct a mathematical model $\mathrm{M}$ that captures these relevant features.

\subsection{Deformations (noncausal interventions)}

In a MEPP, once we implement an optimal representation, the explanandum 'why $\mathrm{P}$ has property $\mathrm{p}^{* \prime}$ becomes 'why $\mathrm{M}$ has property $\mathrm{m}^{* \prime}$. By properly interpreting the answer to the mathematical question we answer the physical question as well. But how should we answer the mathematical question? I believe that not every answer would be explanatory. Recall that at the end of section $\S 15$ I questioned whether all the operations in the derivation step of a scientific explanation are legitimate, and I mentioned that there are cases where the mathematical operations are not explanatory with respect to the physical situation, in the sense that they do not help us to understand why the physical explanandum occurred.

\footnotetext{
${ }^{39}$ Not to be confused with Orzack \& Sober (2001)'s notion of 'optimality models', which are models (mostly found in biology) that describe those traits that maximize fitness (see also Baker (2016) for a description of the cicada case as an optimality model).
} 
Consider the bridges case. A brute computation strategy that proves the impossibility of an Eulerian path over the graph would not provide an explanation of such a result. As we saw, Euler's first solution consisted in trying every possible route (roughly estimated to be 128). If we do this, we will come to the conclusion that none of these routes is an Eulerian path, and for that reason we know that an Eulerian path over the bridges is impossible. This solution, however, does not explain why such a path is impossible (except in the trivial sense that it is impossible because there is no such path available!). Euler's solutions b.2. and b.3 (section §18) did more than just verify the impossibility of such a path. By answering the original question in his Solutio, he also explained why that was the answer to the question. But what makes solutions b.2 and b. 3 explanatory? In this section I argue that these proofs convey counterfactual information about the empirical phenomenon to be explained.

In section §9। introduced Woodward's interventionist account of scientific explanation, according to which scientific explanations exhibit "patters of counterfactual dependence having to do with what would happen under interventions" $(2003,220)$, and in section $\S 15$ I showed how the Inferential Conception of the applicability of mathematics is compatible with this account. Woodward's is a causal account because this counterfactual information is obtained by performing (or thinking of) causal interventions. In a passage of his 2003 book, however, Woodward allows the possibility of explanations that would not appeal to interventions. These would be noncausal explanations:

The common element in many forms of explanation, both causal and noncausal, is that they must answer what-if-things-had-been-different questions. When a theory tells us how $Y$ would change under interventions on $\mathrm{X}$, we have (or have material for constructing) a causal explanation. When a theory or derivation answers a what-if-things-had-beendifferent question but we cannot interpret this as an answer to a question about what would happen under an intervention, we may have a noncausal explanation of some sort (220-221).

Woodward's comments were advanced in the context of a discussion about Mark Steiner's account of explanatory proofs in mathematics (which I examine in section §26). The main point, however, goes beyond Steiner's account. The idea is that what both causal and noncausal explanations have in common is the counterfactual aspect, which may be the main source of explanatory power.

I believe MEPPs belong precisely to this category of counterfactual, noncausal scientific explanations. Given the fact that $M$ optimally represents $P$ with respect to explaining why it has $\mathrm{p}^{*}$, in order to explain why $\mathrm{P}$ has $\mathrm{p}^{*}$ we should obtain information about how $\mathrm{M}$ having $\mathrm{m}^{*}$ depends on $\mathrm{M}$ having property $\mathrm{m}$, which can then be interpreted in terms of property $\mathrm{p}$ of $\mathrm{P}$, and that is how MEPPs obtain their explanatory power:

EXPLANATORY POWER OF MEPPs: By deforming $\mathrm{M}$ with respect to $\mathrm{m}$, we can manipulate whether $\mathrm{M}$ has $\mathrm{m}^{*}$. This procedure can be interpreted in empirical terms, that is, as manipulations of whether $\mathrm{P}$ has $\mathrm{p}^{*}$ by deforming property $\mathrm{p}$, independently of whether it 
is physically possible to change property $p$. Crucially, it is not just $p$, but the whole process ${ }^{40}$ of identifying $\mathrm{p}$ that explains why $\mathrm{P}$ has $\mathrm{p}^{*}$.

In order to explain how we obtain counterfactual information from a mathematical model let me introduce the notion of deformation. As a first approximation, we can think of a deformation as a modification of a property $m$ of a mathematical structure $M$, so that we can manipulate $M$ in a way such that it allows us to answer what-if-things-had-been-different questions about $\mathrm{M}$. Mathematical relations are acausal, so these deformations are noncausal interventions.

Now, this first approximation, though useful, is problematic. Changing an aspect of a mathematical structure might as well count as replacing the whole structure, because mathematical structures have their properties as a matter of necessity. So perhaps these deformations are better understood as replacements of different mathematical structures. The main point remains, though. The idea is that if we replace a mathematical structure by another as close as possible to the first but for that one aspect $m$, we may be able to see which other aspects (e.g. $\mathrm{m}^{*}$ ) must change as a matter of necessity. ${ }^{41}$

These deformations, despite being carried out over a physical system, must not be understood as causal interventions, because although the physical system is composed by elements with causal powers (e.g. wooden bridges), the deformations are not performed taking those causal powers into account (this aspect has also been emphasized by Lange 2013 and Pincock 2007). The reason is that it is not possible, not even in principle, to perform these deformations in the 'surgical' way required by Woodward, that is, as interventions over one property that do not directly affect the explanandum (cf. 2003, 130). $p$ is an element of structure $P$, and by definition this element is related to other elements constitutive of the structure. It is impossible to change this element without altering the others to which it is related.

Now, in a MEPP we use $M$ to optimally represent $P$ with respect to $\mathrm{p}^{*}$. But in order to obtain this explanatory information about how property $p$ of $P$ explains ' $P$ has $P *$ ', we should obtain, for convenience, information about how $M$ having $m$ relates to ' $M$ has $m$ *'. We obtain this information by performing operations in the representation $\mathrm{M}$ that are interpreted in terms of deformations over P. Crucially, these operations on $\mathrm{M}$ do not necessarily have to be understood as explanatory proofs of $\mathrm{M}$ having $\mathrm{m}^{*}$. Whether or not $\mathrm{m}$ explains $\mathrm{M}$ having $\mathrm{m}$ * is not important, because the ultimate goal of a MEPP is to explain an empirical phenomenon, namely, $\mathrm{P}$ having $\mathrm{p}^{*}$. For example, it may well be that $M$ is such that we cannot decide whether $m$ explains $m^{*}$ or in reverse, and so whether one can be used to explain the other is context dependent (see Resnik \& Kushner 1987, and section $\$ 26$ below). This is not an impediment for the physical property represented by $m$ to be explanatory with respect to the physical property represented by $m$ *.

\footnotetext{
${ }^{40}$ This is perhaps the most controversial aspect of my view. I clarify this when I revisit my cases in section $\S 21$, and provide several arguments for it in section 27.2 (b), below.

${ }^{41}$ Pincock (2015) calls these 'Other Object' counterfactuals.
} 


\section{$\S 21$. Cases Revisited}

Let us see how these two notions apply to my cases.

\subsection{The Bridges of Königsberg case revisited}

P: $\quad$ The bridges of Königsberg system (see fig 8)

p*: $\quad$ The property of not allowing paths that cross all the bridges without retracing one's steps (an Eulerian walk)

Physical explanandum: The bridges of Königsberg system does not allow paths that cross all the bridges without retracing one's steps.

M: The bridges of Königsberg graph (see fig 10)

$\mathrm{m}^{*}$ : $\quad$ The property of not allowing an Eulerian path (not-EP)

Mathematical representation of the explanandum: The bridges of Königsberg graph is notEP

First, my account of 'optimal representation' captures what is special about this case. As we have seen, Euler did not represent every single aspect of the system of bridges. The 'geometry of position' he used ignores details such as the actual length of the bridges, the actual distances between the pieces of land, their areas, and even the relative positions of the pieces of land and the bridges. The only features relevant to explaining why an Eulerian path over the bridges is impossible are the number of pieces of land (each piece of land is represented as a node), the number of bridges (each bridge is represented as an edge), and the connections between them. These are the only features relevant to the explanation, and all of them are represented by the mathematical graph. Nothing else needs to be represented.

With respect to the notion of 'deformation', we have seen that its importance lies in its conveying information about the aspects of the physical structure that are relevant to explaining the explanandum. In the explanation, we must identify a property $\mathrm{p}$ of $\mathrm{P}$ that explains the physical explanandum. The explanation is the whole process of identifying a property $\mathrm{m}$ of $\mathrm{M}$ that can be suitably interpreted in terms of $p$. This can clearly be seen in my reconstruction of Euler's solution in section $\S 18$ above.

Now, it is difficult to see how this notion applies to the bridges case in the way it has traditionally been presented in philosophical debates. Euler's solution has usually been summarized like this (note that the premises and the conclusion are mathematical statements):

Let us call the property of a graph having either zero or two odd nodes property SE (for 'semi-Eulerian'), and the property of allowing an Eulerian path, property EP (these properties can be easily understood in terms of concrete road systems): 
P1) The bridges of Königsberg graph does not have property SE

P2) A graph g has EP iff g has SE [mathematical fact]

C) The bridges of Königsberg graph does not have EP

The problem with this traditional reconstruction is that merely knowing that 'A graph $\mathrm{g}$ is EP iff $g$ has SE' does not give us instructions about the aspects of the structure that are relevant for its being non-EP. But we can learn about these aspects if we pay close attention to my reconstruction of Euler's full proof of the theorem, which details the whole process of discovering why not allowing a path that crosses every bridge only once depends on the bridges system having more than two pieces of land connected to an odd number of bridges. Moreover, as we saw, Euler's proof shows how this is in turn related to the fact that a bridge connects two pieces of land. When we pay attention to my reconstruction of the proof, we can see exactly what properties of the structure should be deformed in order to manipulate whether or not the bridges system allow the desired Eulerian walk.

\subsection{The cicada case revisited $d^{42}$}

\section{a) Empirical version of the cicada case}

The first thing we need to do in order to see how my account applies to the cicada case is to separate the empirical explanandum and the mathematical explanandum. By doing this we realize that the life cycles themselves are not prime. Rather, they possess a physical property that is responsible for their evolutionary advantage.

Consider the definitions of the following empirical properties:

1) Iteration of length $L$ : the resulting length of combining successive L's.

$$
I^{n}(L)=L \oplus L \ldots \oplus L \text { (n times) }
$$

2) Overlapping: For any two objects with different lengths $A$ and $B$ respectively, at several points their iterations will have equal lengths ${ }^{43}$ :

$$
I^{n}(A)=I^{m}(B) ; I^{p}(A)=I^{q}(B) ; \text { etc. for some } m, n, p, q \text {, etc. }
$$

3) Overlapping minimization: $I^{m}(L)$ and $I^{n}(L)$ will overlap at $I^{n}\left(I^{m}(L)\right)$; if this is the first time they overlap, then they minimize overlapping with respect to each other.

4) p-coprime: If the only length of which both $I^{m}(L)$ and $I^{n}(L)$ are iterations is $L$, then $I^{m}(L)$ and $\mathrm{I}^{\mathrm{n}}(\mathrm{L})$ are $p$-coprime with respect to $\mathrm{L}$.

\footnotetext{
${ }^{42}$ A version of this section has been published in Barrantes (2017)

${ }^{43}$ This applies to objects with lengths that are rational numbers (on some scale).
} 
5) p-prime: If $I^{n}(L) \neq I^{p}\left(I^{m}(L)\right)$ for any $m ; p<n$, then $I^{n}(L)$ is p-prime with respect to $L$

1-5 are geometrical properties of empirical lengths (including time lengths), not mathematical properties. ${ }^{44}$ This is true despite the fact that, arguably, it would have been very hard (though not impossible) to discover these properties without mathematics. We can now express lemmas 1 and 2 of the number theoretical explanation in terms of these properties:

p-Lemma 1: p-coprime iterations minimize overlapping

p-Lemma 2: If $I^{n}(L)$ is p-prime, then it is co-prime with every $I^{m}(L)<I^{2}\left(I^{n}(L)\right)$

The explanandum in the cicada case is therefore 'why the $I^{13}(L)$ and $I^{17}(L)(L=$ one year) cycles are evolutionarily advantageous'. The explanation depends on the fact that they are $\mathrm{p}$ prime, and as illustrated by the argument in 19.2, it follows that the cycles minimize overlapping, which is evolutionarily advantageous.

b) The cicada case as a MEPP

Now we can see how this case fits my description:

P: $\quad$ The life cycles of cicadas $\left(I^{13}(L)\right.$ and $I^{17}(L)(L=$ one year $\left.)\right)$

p*: Evolutionary advantage

Physical explanandum: Why are the $\mathrm{I}^{13}(\mathrm{~L})$ and $\mathrm{I}^{17}(\mathrm{~L})(\mathrm{L}=$ one year) cycles evolutionarily advantageous?

M: $\quad 13$ and 17

$\mathrm{m}^{*}$ : Minimization of intersection

Mathematical explanandum: Why do 13 and 17 minimize intersection?

First, this explanation relies on an optimal representation. Here the question that worries scientists is why life cycles represented by prime numbers have an evolutionarily desirable property. The mathematical representation which assumes that years are equal to each other captures all the relevant factors for the explanation of this feature of time. Representing years with the natural number system gives us an optimal mathematical model, because more details about the actual time lengths or the causal history the cicadas followed to get to that point are not required. This model captures all and only those features relevant to explaining how the 'overlapping minimization' property is evolutionarily advantageous. What is more, it is our background knowledge about the importance of natural cycles in explaining animal behavior that lead us to take years as the relevant measurement unit, and to idealize years as equal. Once

\footnotetext{
${ }^{44} \mathrm{~m}, \mathrm{n}, \mathrm{p}$ and $\mathrm{q}$ represent natural numbers; but as we will see in section 37.1, using these representations is unproblematic because these numbers can be expressed nominalistically.
} 
years have been idealized as equal, the mathematical explanation cannot be improved (of course, it could be replaced by a different explanation in the face of new discoveries; but the point is that this explanation has all it needs to work as such).

Now, once this model is in place, we can 'forget' about the cicadas and focus on the prime number of years. 'Why are the $1^{13}(\mathrm{~L})$ and $\mathrm{I}^{17}(\mathrm{~L})(\mathrm{L}=$ one year) cycles evolutionarily advantageous?' becomes 'why are prime periods evolutionary advantageous?' The mathematical explanation of this fact explains the empirical question about cicadas only insofar as it shows us how the facts about time explain the life cycles. In other words, we use mathematics to represent time-lengths, and then we use the number-theoretic lemmas to understand why the cycles are evolutionarily advantageous. We see-within the mathematicsthat this is because the cycles are prime; but then we must interpret back this result in empirical terms, thus discovering that it is the overlapping minimization property that explains the evolutionary advantage. The notion of mathematical primeness used to represent $\mathrm{p}$-primeness has surplus structure that we get rid of once we interpret back these results in empirical terms. ${ }^{45}$

\section{$\S 22$. MEPPs as noncausal explanations}

\subsection{In what sense are MEPPs empirical and noncausal?}

It may be argued that MEPPs are causal explanations because MEPPs are empirical applications of mathematics; since their domain is constituted by entities with causal powers, one may think that MEPPs have a causal component. For example, in a recent paper Tim Räz (2014) says that, although not causal, MEPPs are not completely non-causal. This is because "[t]he bridge principle connecting the mathematics and the world is far from trivial; it is an important contribution to [the explanation]" $(2014,19)$. He distinguishes between what he calls the 'Intra Mathematical' (the IME) component of the MEPP, and the 'Scientific Explanation using Mathematics' (the SEM) component of the MEPP. According to him:

[MEPPs] are not purely causal, because the IME component is an explanatory contribution of pure mathematics to these explanations, and mathematics is commonly taken to be non-causal. However, the explanations are not non-causal either. We can interpret the mathematical structure causally $(2014,19-20)$.

For Räz:

Adding the SEM component, the pragmatically motivated bridge principle, turns the IME into an explanation that explains qua interpretation of the structure in terms of causal processes. If the SEM component is removed, the remaining explanation does no longer convey causal information - but it is no longer about the [empirical] system $(2014,25)$.

\footnotetext{
${ }^{45}$ As we saw, this interpretation step is a crucial element of the Inferential Account (e.g. Bueno \& Colyvan 2011, 357; Bueno \& French 2012, 107 and ss).
} 
As I understand it, Räz's point is that MEPPs are not completely noncausal because they include bridge principles that connect the mathematical representation with the empirical system; the interpretation step adds entities with causal powers to the explanation, and for that reason MEPPs are somehow causal (i.e. not completely noncausal).

I think there is a problem with this argument. Every empirical application of mathematics, including MEPPs, must include bridge principles, precisely because these are empirical applications. MEPPs by definition are empirical, that is, they are explanations that explain physical phenomena. We can certainly assert that physical phenomena, in general, have causal powers, so by definition a MEPP is about things with causal powers. But to conclude from this fact that MEPPs have a causal component would make every kind of explanation of physical facts causal. MEPPs indeed involve entities endowed with causal powers, but, as Mark Lange points out, MEPPs do not work "by exploiting those powers" $(2013,485)$, and that is why MEPPs are not causal explanations. MEPPs work by providing information about the physical structures involved, qua structures, but not in virtue of the causal powers of the entities that instantiate those structures. I do not see what is to be gained by labeling these explanations 'not completely noncausal', instead of saying simply that they are about empirical phenomena, which is something already built into the very notion of a MEPP.

\subsection{MEPPs and explanatory asymmetry}

There is a prima facie problem with the suggestion that the explanatory work of MEPPs is carried out purely within the mathematical model. As we have seen (§6), causal dependence was introduced in scientific explanation in order to provide an asymmetry between the explanandum and the explanans. But mathematical relations are not causal, so, is there another way of introducing explanatory asymmetries? This is a problem that must be overcome by any account of mathematical explanations of mathematical facts, but the worry is that this problem may translate to empirical applications, thus undermining the possibility of a project such as mine, that aims to provide an account of mathematical explanations of physical facts.

Consider the flagpole example, which, as we saw (§5), was advanced as a counterargument to Hempel's DN model. Once the light rays, the flagpole, and the shadow are represented geometrically, Geometry by itself does not tell us what explains what, and this is why it is necessary to introduce extra-geometrical considerations. As we saw, causal asymmetries have been the preferred way adopted in the last 40 years of philosophical discussion about scientific explanation. But structural relations (mathematical and empirical) are noncausal; so how do MEPPs overcome the problem of explanatory asymmetry?

First of all, we need to narrow the scope of the task at hand. What we need in a MEPP is for the mathematical derivation to convey some explanatory information about the physical system, regardless of whether this would be explanatory with respect to the mathematical part of the MEPP. In other words, independently of the outcome of the debate about whether there are 
explanatory proofs within mathematics, what we need to solve is the problem of how a structural property $\mathrm{p}$ can be explanatory with respect to another feature $\mathrm{p}^{*}$ of physical system $\mathrm{P}{ }^{46}$

I argue that counterfactual reasoning gives this asymmetry. In the flagpole case, the explanatory dependence is provided by the fact that the length of the shadow depends counterfactually on the height of the flagpole: we can change $e^{47}$ the height of the flagpole in order to manipulate the shadow, but since we cannot do this the other way around, the flagpole explains the shadow, but the shadow does not explain the flagpole's height. Now, in this situation the change in the explanatory property is provided by a causal intervention, and that is why this is a causal explanation. But in other cases this counterfactual information can be provided by other types of changes, for example, by the deformations of the previous section. In that sense, we can capture the explanatory asymmetry without appealing to causal relations.

Let us revisit our cases to see how this idea unfolds. In the bridges case, the question 'why it is impossible to perform a trip over all the bridges of Königsberg without retracing one's steps?' becomes 'why is an Eulerian path over the graph impossible?' This impossibility (a property of the graph) can be manipulated by changing the number of edges (another property of the graph). Remember Euler's two conditions for an Eulerian path (§18):

C1: Either the graph has only two odd vertices, in which case the successful trip must start in one of them;

C2: Or, the graph does not have odd vertices.

The graph representing the bridges of Königsberg does not meet either of these conditions, and that is why an Eulerian path over it is impossible. We can interpret these conditions empirically, and cash out this explanation in terms of the actual system of bridges. Euler's explanation consisted in showing that these two conditions depended on the extremely simple fact that (f): an edge connects two vertices, which corresponds to the physical fact that a bridge connects two pieces of land. As we saw, from the fact that every time one crosses a bridge two letters appear in the path, it follows that, if $K$ is even, then if the journey starts in an even piece of land its letter will appear $\frac{k_{j}}{2}+1$ times in the path sequence, and if the journey does not start in that piece of land, its letter will appear $\frac{k_{j}}{2}$ times in the path sequence; but if $K$ is odd the letter corresponding to that piece of land will appear $\frac{h_{i}+1}{2}$ times, independently of whether or not we start on that piece of land.

This explanation allows us to answer a range of what-if-things-had-been-different questions because we can alter the degree of each vertex $(\mathrm{m})$ and see how the mathematical possibility of performing a Eulerian trip varies accordingly $\left(\mathrm{m}^{*}\right)$, and in that way we can understand exactly how these variations would affect the possibility of performing a Eulerian path. This operation over the graph explains the empirical question about the physical bridges insofar as it shows us how the physical bridges connecting a given piece of land ( $p$ ) explain such impossibility

\footnotetext{
${ }^{46}$ As we will see in section $\S 26$, this is one of the problems of Steiner's account of MEPPs.

${ }^{47}$ I use change as an overarching concept that includes both interventions and deformations.
} 
$\left(p^{*}\right)$, given fact $f$. Euler's proof shows how the impossibility of performing an Eulerian walk ontically depends on $f$. This ontic dependence is captured by counterfactual reasoning. We can use $f$ to manipulate whether a Eulerian walk is possible (e.g. by thinking what would happen if we add more bridges). But nothing that we do to the bridges structure (and its associated properties) would alter $f$. Therefore, the impossibility depends on $f$ but $f$ does not depend on the impossibility, and that is why we can use $f$ to explain the impossibility.

Likewise, in the cicada case the question that worries us is why life cycles represented by prime numbers have an evolutionarily desirable property. As we saw, once we use the natural number series $(M)$ to represent time-lengths, we can use the number-theoretic lemmas to explain why prime numbers $(m)$ minimize intersection with other numbers $\left(m^{*}\right)$, thus explaining why the cycles are evolutionarily advantageous. Changes over the number of iterations (in years) (p) shows us how the system possesses the overlapping property $\left(p^{*}\right)$. Suitable derivations on the mathematical model explain the empirical question about cicadas only insofar as it shows us how the overlapping minimization property depends on two very simple facts about time, namely, that at a certain level of description the notions of combination and equality can be applied to time lengths. Again, the reason why these two notions are explanatory is that the overlapping minimization property depends on combination and equality; but combination and equality do not depend on the overlapping minimization property, and this is the source of the explanatory asymmetry.

I will say more about the problem of asymmetry in section $\S 27$, where I answer a potential objection to my account based on Baker (2012).

\section{$\S 23$. Conclusion}

The main features of my account of MEPPs is that these are scientific explanations that rely on an optimal representation of the target system, capturing the structural features of this system relevant to explaining the explanandum. These structural features are physical relationships between the entities or processes involved, which are then exploited by the MEPP to explain the explanandum. Since the mathematical representation captures all the features that are relevant to the explanation, we can 'forget' about the physical system and work entirely over the mathematical model. Because of this, we can use the mathematical model to acquire counterfactual information about the physical system under question. And although this counterfactual information should be interpreted in empirical terms, we should not interpret it causally. As we have seen, despite MEPPs being non causal, they can account for the explanatory directionality. In that sense, MEPPs are noncausal scientific explanations. In the next chapter, I discuss other accounts of MEPP, and, at the same time, continue clarifying some aspects of my own account of MEPP. 


\section{CHAPTER 4: Other accounts of MEPPs}

\section{§24. Introduction}

Chapter 4. In this chapter, I distinguish between descriptive and representational accounts of MEPPs. The former presuppose the existence of mathematical features in the physical world that are described by the mathematical part of MEPPs; ${ }^{48}$ the latter do not need that assumption, the role of mathematics in MEPPs being to represent the relevant explanatory physical features. In what follows, I will defend my representational account against alternative views of MEPPs.

In section $\$ 25$ I introduce the distinction between descriptive and representational views of MEPPs. Next, in section \$26 I discuss Steiner's view of MEPPs, which is known as the transmission view of MEPPs. Steiner says that at the core of every MEPP there is an explanatory proof of a mathematical theorem. Within the philosophy of mathematics literature, however, the notion of explanatory proof is controversial, and I take it as an advantage of my own view that it does not require explanatory mathematical proofs. In addition, Steiner does not specify which kind of scientific questions may be amenable to be answered by a MEPP. My view does, and that is another advantage over Steiner's. I then argue, against Alan Baker, that MEPPs do require some explanatory information coming from their mathematical component ( $\$ 27)$. Next, my focus is the modal accounts of MEPPs. Aidan Lyon argues that MEPPs appeal to higher-order mathematical features (\$28), and Marc Lange argues that MEPPs point to relations of mathematical necessity in the physical world (§29). I reject these views by pointing out first that they do not specify the sense in which those mathematical features may be explanatory, and secondly, that they do not convincingly establish that there are relations of mathematical necessity in the physical world. Finally, I analyze Christopher Pincock's view of MEPPs as abstract explanations (\$30). For him, MEPPs work in virtue of the mathematical structures underlying the physical world. I show first that the view that mathematical structures may have physical instantiations is unfounded, and secondly that even if this was correct, this view fails to explicate the sense in which MEPPs provide explanatory information.

\section{$\S 25$. Descriptive vs Representational views of MEPPs}

I have argued in the previous chapter that there are mathematical explanations of physical phenomena (MEPPs), which are explanations that rely on optimal mathematical representations of the physical target system. My account is an attempt to make sense of the existence of purely MEPPs in science while preserving mathematics' representational role. But there is of course the Enhanced Indispensability Argument (EIA), which I will thoroughly address in chapter 5. The EIA holds that if there are MEPPs, then we ought to be committed to mathematical realism. I admit it may be puzzling to learn that mathematics is merely representational and that there are mathematical explanations in science. In order to clarify this, I will use a distinction due to James Robert Brown (2008). For Brown, there are two ways of understanding the relationship between the mathematical realm and the physical realm. Either these two realms are separate, and in

\footnotetext{
${ }^{48}$ Bueno \& French call this idea that some properties are physico-mathematical the 'hybridity claim' (see e.g. 2017, 141).
} 
applications mathematics represents the physical realm; or these two realms are not completely separated, and in applications mathematics describes mathematical features of the physical world. According to Brown, this distinction is pertinent to the indispensability debate. For example, he uses it to understand the differences between views like Field's and views like Quine and Putnam's:

Looking back on the debate, Field versus Quine and Putnam, we can see it as an implicit debate about whether mathematics represents (Field) or describes (Putnam and Quine). I think of Field as being on the representational side since he has explicitly used the results of measurement theory as done in the representational way. My reason for saying Quine and Putnam see mathematics as describing the world stems from their various remarks about the possibility of revising mathematics and logic in the face of experience. On the representationalist (or modeling) view of applied mathematics, this would be absurd, since an empirical upset would simply make us look for a different mathematical model to represent things; it would not lead us to change our mathematical theories themselves (Brown 2008, 61).

Although the descriptivist view is committed to some sort of mathematical realism, the representationalist view is not necessarily committed to nominalism, despite the fact that Brown cites Hartry Field (a nominalist) to illustrate this view. A mathematical Platonist may agree that the physical and the mathematical realms are separated, but still believe that mathematical statements quantify over real, non-physical entities (Brown himself endorses this view (see e.g. 2008, 156)).

Now, although the distinction between representation and description is not thoroughly discussed in Brown's book, I believe it will be helpful for understanding the sense in which my view of MEPPs is different from most accounts of MEPPs in current literature. Mine is a representationalist view, because I hold that even in MEPPs the role of mathematics is to represent physical features. I believe that most of the accounts of MEPPs that I will discuss can be understood under the 'descriptive view' (Steiner's is an exception). Alan Baker, for example, holds that MEPPs apply directly to the mathematical features of the physical explanandum; Aidan Lyon argues that MEPPs appeal to higher-order mathematical features of this explanandum; Marc Lange says that MEPPs point to relations of mathematical necessity in the physical world; and finally, Christopher Pincock argues that MEPPs work in virtue of the mathematical structures underlying the physical world. All these accounts highlight important features of MEPPs. I will show that my view also has these features, but without appealing to alleged mathematical properties of the physical explanandum.

\section{§26. Mark Steiner: MEPPs and mathematical explanations}

\subsection{Steiner's view of MEPPs}

One of the first analyses of the structure of MEPPs was given by Mark Steiner (1978b). According to him, MEPPs are scientific explanations whose explanatory power depends on an explanatory proof of their mathematical component. Steiner's account depends on the existence of mathematical explanations of mathematical facts, so let me say something about this debate. 


\section{a) Explanatory and non-explanatory proofs}

According to many philosophers of mathematics, there is a difference between proofs that merely verify the truth of a theorem and proofs that explain the theorem. ${ }^{49}$ Non-explanatory proofs show that a given theorem is true. Explanatory proofs show, in addition, why the theorem is true. This view is shared by some mathematicians. For example, Giancarlo Rota says that:

Not all proofs give satisfying reasons why a conjecture should be true. Verification is proof, but verification may not be reason (1997, 186-7; quoted in Mancosu 2001, 100).

In the same spirit, Timothy Gowers and Michael Neilson say that:

[P]roofs are more than guarantees of truth: they are valued for their explanatory power, and a new proof of a theorem can provide crucial insights $(2009,879$; quoted in Colyvan $2012,78)$

Let me illustrate what this distinction may be with an example. Imagine that we want to prove the following theorem:

Every even natural number between 4 and 200 can be expressed as the sum of two primes (cf. Baker 2009a)

Evidently, we can show that the theorem is true if we verify it for every element in the domain, but it should be clear that we do not understand why the theorem holds; we do not understand the relation between evenness, primeness, and addition that makes the theorem true. This particular kind of brute computation strategy is called proof by exhaustion. In an explanatory context, these proofs are useful for establishing the truth of the explanandum, and as we have seen, a true explanandum is a requisite for a successful explanation. But evidently, establishing the truth of the explanandum is not necessarily the same as explaining it. Other kinds of proofs that are deemed to be non-explanatory are reductio proofs (Colyvan 2012, 79), and proofs by induction (Lange 2010), although this has been disputed (Baker 2009a).

Now, as a matter of fact, there is no consensus in the philosophy of mathematics about what exactly the distinction between explanatory and non-explanatory proofs amounts to. In addition, there are many cases of proofs where intuitions disagree on labeling them as explanatory or not. I will not fully address this discussion here. In order to understand Steiner's account of MEPPs, we only need to examine his notion of mathematical explanation.

\footnotetext{
${ }^{49}$ See Steiner (1978a); Mancosu (2001), (2010); Sandborg (1998); and Colyvan (2012.) For a contrary view, see Resnik \& Kusher (1987) who argue that there are no mathematical explanations simpliciter. For them, mathematical explanations are context-dependent (more on this below).
} 
b) Steiner's counterfactual account of explanatory proofs

According to Steiner, explanatory proofs are those that:

make reference to a characterizing property of an entity or structure mentioned in the theorem, such that from the proof it is evident that the result depends on the property (1978a, 143; my emphasis).

Steiner uses the notion of characterizing property in order to avoid the expression 'essential property'. An essential property, Steiner argues, is a property an entity possesses in all possible worlds. But since all mathematical truths are necessary, a mathematical entity would possess all its properties as a matter of necessity, which implies that every property of a mathematical entity would be essential. For Steiner, using this term would confuse the meaning of the definition $(1978$ a 143.) A characterizing property, on the other hand, is "a property unique to a given entity or structure within a family or domain of such entities or structures" $(1978 a, 143$.) The notion of 'family of structures', Steiner explains, is relative, in the sense that "a given entity can form part of a number of different domains or families." $(1978 a, 143){ }^{50}$ The idea is that the proof must deduce the theorem to be proved from this characterizing property, in such a way that, if the characterizing property were different, then the theorem would not have held. For Steiner, this counterfactual information can be used to explain facts about such an entity:

[In an explanatory proof] it must be evident... that if we substitute in the proof a different object of the same domain, the theorem collapses; more, we should be able to see as we vary the object how the theorem changes in response. In effect, then, explanation is not simply a relation between a proof and a theorem; rather, a relation between an array of proofs and an array of theorems, where the proofs are obtained from one another by the 'deformation' prescribed above. (But we can say that each of the proofs in the array 'explains' its individual theorem) (1978a, 143; my emphasis).

Steiner provides the following example: We can prove the Pythagorean Theorem by appealing to the mathematical fact that the areas (A) of similar figures are to each other as the squares of their corresponding sides (see equation (a), below), and to the fact that a right triangle can be divided into two triangles that are similar to it (see equation (b), below).

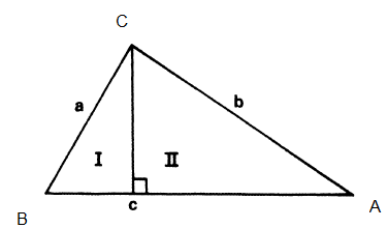

fig. 19.

\footnotetext{
${ }^{50}$ As we will see, this is a big problem for this view.
} 


$$
\frac{A_{I}}{a^{2}}=\frac{A_{I I}}{b^{2}}=\frac{A_{I+I I}}{c^{2}}=K \ldots .(a)
$$

where $K$ is a positive real. From (a) it follows that

$$
A_{I}=K a^{2} ; A_{I I}=K b^{2} ; A_{I+I I}=K c^{2} .
$$

But given that:

$$
A_{I}+A_{I I}=A_{I+I I} \ldots(\mathrm{b})
$$

we have that

$$
K a^{2}+K b^{2}=K c^{2}
$$

from which the Pythagorean Theorem follows.

According to Steiner, this proof is explanatory because it appeals to a characterizing property of right triangles, namely, the right angle, and this property plays a crucial role in the proof. It is easy to see, as we perform hypothetical 'deformations' of this angle, that no non-right triangle can be divided in two parts such that they are similar to each other and to the original triangle (cf. 1978a, 144) (see fig. 20). This can only happen with right triangles when the dividing segment is the height relative to the hypotenuse (compare fig. 19 with fig 20).

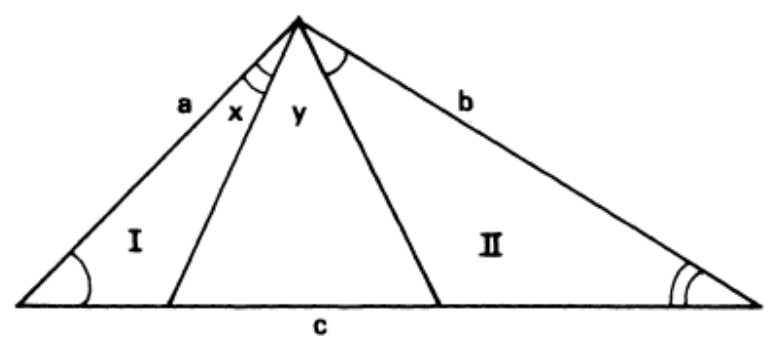

fig. 20 (1978a, 144)

The proof depends on the fact that the right triangle is the only one that can be divided in two triangles similar to each other and to the whole (cf. 1978a, 144). So, equation (b) in the proof depends precisely on the property of having a right angle. Since (b) is crucial for the proof to work, for Steiner this would be an explanatory proof.

Now, there are other proofs of this theorem, but only those that are based on a proper characterization of the relevant properties that make a right triangle what it is would count as explanatory. 


\section{c) MEPPs as mathematical explanations}

With this account of explanatory proofs, Steiner develops an account of MEPPs. According to him, there are three elements involved in a MEPP: the physical explanandum $P^{\prime}$, a mathematical explanandum $\mathrm{M}^{\prime}$ of $\mathrm{P}^{\prime}$, and a mathematical explanans $\mathrm{M}^{51}$ Given some bridge assumptions, the physical explanandum $P^{\prime}$ is represented by the mathematical explanandum $M^{\prime}$, which in turn is explained by the mathematical explanans M. The MEPP should include these three elements, and it inherits its explanatory power from the explanation of $\mathrm{M}^{\prime}$ by $\mathrm{M}$. Schematically the relation is like this:

\section{$M \rightarrow M^{\prime}-P^{\prime}$}

fig. 21.

Adapted from Baker 2012, 247.

The arrow ' $\rightarrow$ ' represents an explanation, and the dash ' - ' represents the set of bridge principles linking $\mathrm{M}^{\prime}$ and $\mathrm{P}$ '. For Steiner, at the core of a MEPP there is always a 'pure' mathematical explanation. On this view, the special feature of MEPPs is that if "we remove the physics, we remain with a mathematical explanation - of a mathematical truth" (1978b, 19), as opposed to ordinary scientific explanations, in which "after deleting the physics nothing remains" (1978b, 19). The idea is that it is by working over the mathematical explanandum that it is possible to find an explanation that can then be transmitted to the physical explanandum P'. Alan Baker calls this account "the transmission view" $(2012,246)$, because the explanatory power of the explanation comes from the mathematical explanation of the mathematical fact, which is then 'transmitted' to the explanation of the physical fact. In MEPPs, once the explanation of $\mathrm{M}^{\prime}$ has been found, nothing explanatorily relevant is added when one applies the explanation to phenomenon $\mathrm{P}^{\prime} .^{52}$

\subsection{Problems with Steiner's View}

There are some problems with Steiner's account of explanatory proofs. First, as Resnik \& Kushner point out, it is not clear how explicit the 'mentioning' of the characterizing property must be. A proof may rely on a characterizing property without mentioning it explicitly. Would that proof be explanatory? It seems fair to assume that it would be, but Steiner seems to think otherwise. For example, he rejects proofs by induction as non-explanatory because, although they "characterize the set of natural numbers", this set "is not mentioned" in the proof (1978a, 145). But it seems that whether or not this characterization is explicitly mentioned, or whether it is evident that the

\footnotetext{
${ }^{51} \mathrm{M}, \mathrm{M}^{\prime}$, and $\mathrm{P}$ refer to mathematical and physical structures respectively.

52 In the same vein as Steiner's, Mark Colyvan calls MEPPs 'extra-mathematical explanations'. These are 'intra-mathematical explanations' that "spill over' into physical applications" $(2012,90)$. He provides the following condition for an explanation to be a MEPP: "[C]onsider an explanatory proof of some mathematical theorem. If that theorem has some physical application, then the proof of the theorem might well explain what's going on in the physical situation" $(2012,90)$. In these cases, says Colyvan, "it seems that mathematics is carrying the bulk of the explanatory load" $(2012,91)$. My criticisms of Steiner's view apply to Colyvan's view as well.
} 
proof depends on it, are pragmatic issues that should not determine whether the proof is explanatory.

Another problem is that some non-explanatory proofs can indeed be deformed in the way suggested by Steiner, and some explanatory proofs cannot. For example, an inductive proof of the fact that the product of every three consecutive integers is divisible by 6 can be 'deformed' into an inductive proof of the fact that the product of every four consecutive integers is divisible by 24 (see Lange 2014, 521). But, arguably, this proof is not explanatory (Steiner himself would agree that this is not an explanatory proof, although, as I have argued, for the wrong reasons). One reason why this proof may not be explanatory is that it does not tell us what property of these series of three numbers makes them divisible by 6 . On the other hand, there are explanatory proofs that cannot be deformed. For example, the standard proof of the intermediate value theorem is generally taken to be explanatory, despite the fact that the same 'proof idea' cannot be applied to non-continuous functions (Resnik \& Kushner 1987, 146-149). Therefore, whether or not a proof can be deformed in the way Steiner suggests does not determine whether or not the proof is explanatory.

Another case where deforming the proof into a different one of the same 'family' seems to be irrelevant to its status of explanatory as such, is Euler's proof of the graph theoretical theorem mentioned in the bridges case. The proof works by defining a general connected graph, and uses the fact that it is a connected graph to calculate the total number of nodes that will appear in the path $(n+1$; step 7 of the proof in section $\$ 18)$ (how explicit this characterization is, is unclear to me). We take this proof to be explanatory, however, there is no explicit mentioning of how it would work for non-connected graphs. If we think of a specific non-connected graph, it is hard to see how the same 'proof idea' could be applied. There is no equivalent of step 7 in a general proof for non-connected graphs (in fact, the very idea of asking for a continuous walk on a non-connected graph does not seem to make sense). But despite this, Euler's proof strikes us as explanatory. Why? Because it deduces the theorem from basic facts about even and odd nodes, which in turn are based on the simple fact that an edge connects two nodes. In the proof, we can see how these elementary properties are responsible for the theorem.

Now, I just mentioned that the proof of the intermediate value theorem and Euler's proof 'strike us' (strike me?) as explanatory, whereas proofs by induction do not. But how can I say this without providing an account of explanatory proofs? This is a complicated debate, and I do not want to take sides, especially because intuitions differ among mathematicians as to which specific proofs are or are not explanatory. It is hard to determine a set of cases that must be accommodated by all accounts of explanatory proofs. I believe some proofs are explanatory when they show how the theorems depend on some of the properties of the entities involved, but this does not mean that deforming those properties would provide us with alternative proofs of the same family. I will not thoroughly defend this view here because I do not think I have to. In fact, I take it as a shortcoming of Steiner's view (and of other transmission views that depend on explanatory proofs, such as Colyvan (2012)) that it depends on the outcome of this debate. My account of MEPPs does not rely on a specific view of mathematical explanation. As I have shown, what we need in a MEPP is for the mathematical derivation to convey some explanatory 
information about the physical system, regardless of whether this information would be explanatory with respect to the mathematical part of the MEPP.

In addition, Steiner's view is unsatisfactory as an account of scientific explanation. First because transmission views that depend on explanatory proofs may not be capable of accounting for the explanatory directionality in some cases. A deduction of a theorem from the axioms preserves an asymmetric dependence, i.e. the axioms are more fundamental than the theorem. But not all proofs work this way, and it is sometimes not possible to determine which property is more fundamental than another, precisely because, as Steiner points out, mathematical structures have their properties as a matter of necessity (all properties are 'essential' to the structure). Steiner tried to solve this problem by appealing to the notion of characterizing property, but objectively identifying a characterizing property is not always possible. ${ }^{53}$ In the bridges graph, for example, it may seem that the fact that the graph does not allow Eulerian paths is a byproduct of the fact that the graph is non Eulerian. But the structure may as well be characterized as non Eulerian because it does not allow Eulerian paths. It is unclear that one characterization is more fundamental than the other. But despite this, as we have seen, in terms of the concrete system of bridges, features of the bridges structure explain the impossibility, but not the other way around. It is precisely because MEPPs are about concrete systems that they do not have 'the problem of asymmetry', in a way that explanatory proofs may have (I will say more about this problem in 27.2, below).

Finally, another problem with Steiner's view, the most serious one because it directly relates to how MEPPs are supposed to work as scientific explanations, is that Steiner does not specify the relationship between the physical explanandum $P^{\prime}$ and the mathematical representation $\mathrm{M}^{\prime}$. He says that, given some assumptions, $\mathrm{P}^{\prime}$ is represented by $\mathrm{M}^{\prime}$, which in turn requires an explanatory proof. But he does not say anything about which $\mathrm{P}^{\prime}$ s are suitable to be explained by a MEPP. Why is a MEPP needed to explain a given physical phenomenon $\mathrm{P}^{\prime}$ in some cases but not in others? Steiner does not say. As I have shown, by introducing the notion of optimal representation, my account gives more details about which cases should be explained by a MEPP: given our background knowledge about what is relevant to explaining a physical phenomenon, we may or may not implement an optimal mathematical representation. If we do, then we will have to use a MEPP.

Ultimately, my view can be understood as a variation of Steiner's. As we have seen, according to Steiner, a MEPP is an explanation whose explanatory power comes from a mathematical explanation of the mathematical facts featuring in the explanans, such that "if we remove the physics, we remain with a mathematical explanation - of a mathematical truth" $(1978 b, 19)$. On this view, when we eliminate the physical component of a MEPP, all that is left is a mathematical explanation. My account emphasizes the other side of the coin. On my view, in a

\footnotetext{
${ }^{53}$ In fact, as I mentioned in fn. 49, Resnik \& Kushner argue that there are no explanatory proofs simpliciter: "[N]othing is an explanation simpliciter but only relative to the context-dependent why question(s) that it answers" $(1987,153)$.
} 
MEPP if we were to 'remove the mathematics', we remain with an ordinary scientific explanation of an empirical truth, the only difference being that it would be a noncausal explanation.

\section{§27. Alan Baker: MEPPs, Indispensability and 'bare' mathematical truths}

\subsection{Baker's Science-Driven Mathematical Explanations}

a) Indispensability and non-arbitrariness

On Baker's view, for a scientific explanation to be counted as a MEPP, it is not enough that it has a mathematical component. The mathematical component must be explanatory in its own right, and not merely be a representational device. For Baker, therefore, in a MEPP mathematics plays an explanatory role in addition to its representational role. Now, although Baker acknowledges that it is difficult "to demonstrate that the mathematical component is explanatory" $(2009,625)$, he proposes that one way of doing this is by showing that the mathematical element involved is indispensable in the explanation, by which he means that it is not arbitrary.

Let me illustrate this with an example of my own. A piece of mathematics that plays an arbitrary role would be the number that corresponds to the boiling point of water in normal conditions. If we use Celsius as our temperature unit, the boiling point would be 100; but if we use Fahrenheit as our temperature unit, it would be 212. The idea is that nothing hangs on this choice of units. No property of water would be picked out by a property of either of those numbers because the choice of those numbers relies on an arbitrary convention:

The thought is that if it can be shown that the choice of mathematical apparatus is just one of many equally good alternatives then the particular mathematical objects involved cannot be indispensable to the overall explanation (2009, 614-15).

On the other hand, if the mathematical apparatus is not arbitrary, then it is indispensable, and on Baker's view, explanatory. This is why, for example, for Baker the cicada case is a MEPP:

I think that the cicada explanation is well placed to meet charges of arbitrariness [...] The units involved in the explanation arise from intrinsic physical features of the situation, the number-theoretic notion of primeness plays a key role, and-despite the relative simplicity of the mathematics involved-no easy nominalistic paraphrases are available $(2009,619)$.

On Baker's account, a physical phenomenon requires a MEPP when it can only be represented by a specific mathematical property. This is because the explanatory property is a mathematical property of the physical system. In other words, what carries the explanatory load is the link between the physical properties and the mathematical properties of the physical system involved. This 'hybridity' between the physical and the mathematical realm is a view shared by descriptive accounts of MEPP. ${ }^{54}$ In the cicada case, for example, the concept of primeness is not merely representing a property of time lengths: for Baker, the time lengths themselves are prime (cf. 2009, 621). The cicadas' life-cycles have the property of primeness; truths related to this

\footnotetext{
${ }^{54}$ I take this notion of hybridity from Bueno and French (2017).
} 
property (the number theoretical lemmas) explain why having this primeness property makes the cycles evolutionarily advantageous, which in turn explains why cicadas evolved life cycles having this property. Put this way, realism of mathematical properties is a condition for there being genuine MEPPs in science. This hybridity claim is also endorsed by Lange (2013) and Pincock (2015).

\section{b) 'Bare' mathematical results}

In his (2012), Alan Baker argues that in MEPPs (what he calls 'Science-Driven Mathematical Explanations': SDME) we do not need to explain the mathematical truths cited in the explanans in order to have an explanation of physical facts (Pincock (2015) and Lange (2002) also endorse this). The idea is that as long as it is known that a given theorem is true, it can successfully be used in empirical applications:

In general, all scientists need to know when they appeal to a given mathematical result in the context of a science-driven mathematical explanation is that this result has been proved. Nothing else about the proof matters for the purposes of the overall SDME, nor does the proof need to be included in the presentation of the SDME (Baker 2012, 263).

For Baker, the mathematical facts cited in the explanans of a MEPP have the same status as the initial conditions in an ordinary scientific explanation, which are just assumed to be present in the situation to be explained, but do not need to be explained themselves. Baker tracks back this idea to Hempel, who said that "an explanation can be completed even if the facts to which it appeals are left unexplained" (quoted in Baker 2012, 263). For example, we do not need to explain why there are raindrops in the air in order to explain the rainbow. In the same way, Baker argues, we do not need to explain the mathematical facts that we use in a MEPP. Rather, what a MEPP does is to show how, given the initial conditions and the mathematical facts, the explanandum follows. The idea is that the physical explanandum of a MEPP gets explained when one finds truths about its mathematical properties, but the means by which one finds these truths are not an essential component of the explanation.

In the cicada case, for example, the proof of the two number theoretical lemmas is not relevant for explaining the lengths of the cicada's life-cycles:

$[T]$ he proofs of these two lemmas - while relatively elementary - were not presented or discussed; instead readers were referred, without further comment, to Edmund Landau's Elementary Number Theory... This suggests that even a more fleshed-out version of the cicada [MEPP] need not contain any mathematical proofs (Baker 2012, 262).

On Baker's view, therefore, a proper reconstruction of the cicada case would be like this:

1a) The lowest common multiple of $m$ and $n$ is maximal iff $m$ and $n$ are coprime [Lemma 1] 
2a) A number $\mathrm{m}$ is coprime with each number $\mathrm{n}<2 \mathrm{~m} ; \mathrm{n} \neq \mathrm{m}$ iff $\mathrm{m}$ is prime [Lemma 2]

3a) Prime numbers minimize intersection with numbers less than their double $(1 a+2 a)$

4a) The life cycles have the mathematical properties of the numbers that represent them [hybridity]

5a) Prime life cycles minimize intersection with cycles less than their double $(3 a+4 a)$

6a) Prime numbered life cycles contribute to minimizing the possibilities of intersection with other species that lessen the fitness of the given species (5a)

7a) Features that contribute to minimizing the possibilities of intersection with other species that lessen the fitness of the given species are evolutionarily advantageous

8a) Prime numbered life cycles are evolutionarily advantageous (5a+6a)

In the same way, the bridges case would be reconstructed like this:

1b) The bridges of Königsberg graph does not have property SE

2b) A graph $g$ is EP iff $g$ has SE [mathematical truth]

3b) The bridges of Königsberg graph is not-EP $(1 b+2 b)$

4b) The bridges graph and the bridges system share the same structure [hybridity]

5b) The bridges of Königsberg system does not allow continuous walks over all its bridges without retracing one's steps $(3 b+4 b)$

On Baker's view, it is not necessary, for explaining the conclusion, to provide an explanation of $(1 a, 2 a)$ and $(2 b)$ respectively. This is a rejection of the 'transmission view', including my version of it. In the bridges case, for example, Baker's view requires that we ignore steps (1) to (13) of my reconstruction (see $\S 18$ above), which I think are essential for the overall explanation to work as an explanation. 


\subsection{Problems with Baker's view}

\section{a) The indispensability of mathematics}

The whole of chapter 5 is devoted to the alleged indispensability of mathematics in science, and section $\S 38$ is specifically focused on the different ways in which mathematics can be indispensable in MEPPs, but the main points can be presented here. I will argue that, although mathematics can be indispensable to have cognitive access to the relevant explanatory physical features, this does not entail that a mathematical representation is the only way one can have access to these explanatory physical features, let alone that these explanatory features are themselves mathematical.

In the bridges case, for example, in principle one can discover the relevant structural facts about the bridges system by observing the bridges system from above. In the same way, we can conceive of some alternative means one may have discovered the relevant facts about time that operate in the cicada case, deducing them from the facts about combination and equality. Mathematics is said to be indispensable only because, though conceivable, it is not plausible that one may actually come to know the facts about road systems or life-cycles without mathematics. Mathematics makes it plausible to discover those modal facts in the physical world, but once we envision a way to discover these facts, the use of mathematics becomes merely pragmatic. As we will see, this usefulness of mathematics in science does not support the view that the physical world has mathematical properties.

\section{b) The case for explanatory derivations}

In my reply, I will focus on the bridges case, but everything I say about it applies to the cicada case as well.

\section{b.1. Mathematical truths are not initial conditions}

Contrary to Baker's view, I believe that in a scientific explanation, mathematical truths do not play the same role as the initial conditions. The role of mathematics is either to represent these initial conditions, or to track down the relationship between the initial conditions and the explanandum. As we have seen, Hempel's D-N model also assigned a representational role to mathematics. In the $\mathrm{D}-\mathrm{N}$ model, deduction is the relevant explanatory tool, mathematics being just a tool to carry out these deductions (c.f. the 'theoretical juice extractor' metaphor). In a similar way, for Wesley Salmon, the role of mathematics consisted in describing spatiotemporally continuous causal processes and interactions linking the explanandum to these initial conditions; and for James Woodward, mathematics should track down the mechanisms linking the two (see section §15).

In MEPPs, we are not describing causal relationships, but we still want to know how the relevant aspects of the structure are responsible for the explanandum. This is what is going on, for example, in Euler's explanation in the bridges case. Here we want to know, given the bridges graph, why it does not allow an Eulerian path. In order to learn this, we must spell out the reasons why (2b) holds. It is not enough to say that it holds. This is true independently of whether the 
hybridity claim is true. That is, even it if is true that physical structures are, intrinsically, mathematical structures, we still need to say something about why the said structure has its properties. That is, regardless of whether (2b) is a description of genuine mathematical features of the world (the 'mathematical structure' of the bridges system), merely citing (2b) is not enough to have an explanation. An explanation must show how the explanandum depends on some properties of the structure.

\section{b.2. The problem of relevance}

The failure of Hempel's D-N model has taught us that merely showing how the explanandum is a logical consequence of the facts cited in the explanans does not constitute an acceptable explanation. This is because this deduction by itself does not inform us about the features relevant to the occurrence of the explanandum. For example, in order to explain why a given animal is black, it is not enough to say that it is a raven and that 'all ravens are black'. Rather, we must show, as Woodward puts it, what features of ravens are responsible for their blackness; that is, how blackness counterfactually depends on some specific features of ravens, such that, if those features were altered in different ways, we would be able to manipulate the raven color. 'All ravens are black' can be used to show that the explanandum was to be expected, but it "doesn't tell us anything about how to change the color of a raven $a$ or any other raven or bird" $(2003,193)$. 'All ravens are black', therefore, does not inform us about features of ravens relevant to their blackness.

In the same way, if we do not know the sense in which some features of the bridges structure are associated with the property of not allowing an Eulerian walk, the argument above would only be establishing the truth of the explanandum, but it would not be an explanation of it. What makes the bridges of Königsberg system fail to allow an Eulerian walk? If the argument does not answer this, it is not an explanation. Merely citing 'a graph g has EP iff g has SE', as Baker would suggest, does not inform us about features of the structure relevant to its not allowing an Eulerian path. Contrary to this, Euler's proof shows how specific aspects of the bridges system structure relate to its not allowing an Eulerian path. Specifically, it shows that the fact that a bridge connects two pieces of land is responsible for the relevant distinction between 'odd' pieces of land, and 'even' pieces of land, which in turn relates to the possibility (or lack thereof) of an Eulerian walk.

\section{b.3) Levels of explanation and Scientific Practice}

Now, it could be argued that, by altering the variables cited in (2b), we can indeed use (2b) to manipulate the impossibility of performing an Eulerian path. In that sense, we do not need an additional explanation for why (2b) holds. This defense of Baker's view relies on a distinction between levels of explanation. At one level, we have the explanation of (5b); at a different level, we have the explanation of (2b).

The problem with this approach is that it assumes a distinction between explanations of regularities, which would appeal to causes, mechanisms, or structural relations, and explanations of particulars, which would be performed by deduction from these generalities, in the way proposed, for example, by the D-N model. But as Woodward has argued, scientists do not usually 
make a distinction between explanations of generalizations, and explanations of particulars. For example, whether they want to explain why a is black or why all ravens are black, they will appeal to the same genetic and biological mechanisms:

The "two-level" approach thus has the implication that there are two quite different varieties of explanation that work according to different principles, one having to do with explanation of regularities and the other having to do with the explanation of singular explananda. $(2003,391)$

In other words, the two-levels of explanation view would entail that deduction from regularities explains particular events, whereas only generalizations are explained by appealing to the relevant mechanisms. This, Woodward argues, is not found in scientific practice:

[I]n many areas of science, explanations typically or commonly take the form of explanations of generalizations or regularities, with the explanation of particular outcomes being parasitic on this activity, in the sense that it draws on the same information (2003, 391).

\section{b.4) The Asymmetry Problem}

The most serious problem with Baker's view that MEPPs work by appealing to 'bare' mathematical truths, is that if that were the case, MEPPs would not be able to account for the explanatory directionality. In the bridges case, for example, the view that citing ( $2 b$ ) is all we need to provide an explanation entails that the following argument would also count as an explanation:

$\left.P 1^{*}\right)$ The bridges of Königsberg graph is not-EP

P2) A graph s is EP iff a has SE

$\left.C^{*}\right)$ The bridges of Königsberg graph does not have property SE (1+2)

This is what Craver \& Povich call 'a reverse case', that is, an alleged scientific explanation that fails to account for the explanatory asymmetry between the explanandum and the facts cited in the explanans $(2017,33)$. As we have seen (\$3), the most famous example for illustrating the failure of Hempel's D-N model in accounting for explanatory asymmetries is the flagpole case. On Craver \& Povich's terminology, the length of the shadow explaining the height of the flagpole would be the reverse case of the (correct) explanation, which uses the height of the flagpole explaining the length of the shadow.

The same happens in the bridges case. The reason why the reverse bridges case is not correct is that whether or not a system has bridges connecting in a certain way depends on other considerations, such as the reasoning of the people who decided to build the bridges, and not on whether or not a particular walk is possible (Craver \& Povich 2017, 34). When we ask about why the Königsberg system fails to have either zero or two pieces of land connected by an odd number of bridges, we are demanding why the system was built with this specific structure. On the other hand, when we ask why the system does not allow an Eulerian walk, we are asking about a 
property the system has in virtue of having the structure it has. We can use facts about the structure to explain why the system is not Eulerian walkable, but we cannot use the fact that it is not Eulerian walkable to explain why it was built in a particular way.

As I have argued (\$22), Euler's proof makes it clear how this explanation accounts for the asymmetry between the explanandum and the explanans. Euler showed how the impossibility of performing an Eulerian walk depended on the bridges structure, because it ultimately depended on the fact that a bridge connects two pieces of land. We can use this fact about bridges to manipulate whether an Eulerian walk is possible. But nothing that we do to the bridges structure (and its associated properties) would alter the fact that a bridge connects two pieces of land. Therefore, the impossibility depends on this fact about bridges, but this fact about bridges does not depend on the impossibility, and that is why we can use this fact about bridges to explain the impossibility. We can only learn this information if we disaggregate ( $2 b)$ in terms of the reason why such generalization obtains.

\section{$\S 28$ Aidan Lyon: MEPPs and program explanations}

An aspect of MEPPs that has often been highlighted in the literature is their modal component. ${ }^{55}$ The idea is that MEPPs show that the explanandum was somehow bound to happen, independently of its actual causal history. We have already seen that many ordinary scientific explanations have this feature as well (section §8). Let us examine now how MEPPs provide this modal information, and if the modalities involved in MEPPs are stronger than those of ordinary scientific explanations. In the following two sections I will examine two modal accounts of MEPPs, Aidan Lyon’s (\$28) and Mark Lange’s (\$29).

Lyon holds that MEPPs are program explanations. Recall (§8) that Jackson \& Pettit (1990) call 'program explanations' those that do not depend on citing the specific causal processes that produced the explanandum, but on higher-order properties that ensured (or, as they put it, 'programmed') that there will be some set of causal processes that will be efficacious in producing the explanandum. These higher-order properties are not causally efficacious in producing the explanandum, but they are causally relevant precisely because they play this programming role. The explanatory strength of these program explanations comes from citing these 'programming properties' because they show that the explanandum is modally stronger than it could have been rendered by the specific causal processes that actually produced it.

This seems to resonate with the examples I have discussed so far. In the bridges case, whereas process explanations would show why in any particular attempt an Eulerian path could not be completed, by appealing to the overall structure of the bridges system the MEPP shows that "no matter how anyone chose to go for a walk around Königsberg, they would never pass over each bridge exactly once" (Lyon 2011, 10). Similarly, even though in principle a detailed process explanation could be given of why the cicadas have life cycles of 13 and 17 years, that explanation "misses the fact that the final evolutionary outcome, the convergence on 13 and 17, is robust with respect to the historico-ecological details" (Lyon 2011, 9). By appealing to higher

\footnotetext{
${ }^{55}$ See for example Baker 2005; 2009; 2012; Colyvan 2001; 2012; Lyon 2011; Lange 2013; Pincock 2015.
} 
order properties of the time lengths the MEPP shows that even if some of those historicoecological details had been different, the cicadas would still have had such life cycles.

Now, according to Lyon's account, what these examples have in common, in addition to being program explanations, is that the higher-order property doing the 'programming' is mathematical. And this is, according to him, the distinctive feature of MEPPs:

An explanation of an empirical fact is mathematical - i.e. it has mathematics doing explanatory work - if the explanation is a program explanation that uses mathematics in a way that is indispensable to the program. Take away the mathematics and the program falls apart $(2011,10)$.

On this view, mathematics does genuine explanatory work when it does the programming work in a program explanation. Lyon associates this with mathematical realism $(2011,559)$. It is because the physical system has this higher-order mathematical property that a lower-level property produced the explanandum. We know that a mathematical property is doing the programming work when, as Lyon puts it, it is 'indispensable to the program'. It is in that sense that MEPPs show that the explanandum is modally stronger than the causally efficacious processes that actually produced it.

\subsection{Problem with Lyon's view}

Now, a serious charge against Lyon's view is that the role of mathematics in program explanations may be taken to be simply representing the higher-order nominalistic property that does the real explaining. This problem has also been pointed out by Juha Saatsi:

"[In these examples] it is not clear why the higher-order property doing the programming should be viewed as a mathematical property. That is, it is not clear why mathematics cannot be viewed as playing a broadly representational role vis-à-vis some nominalistically acceptable higher-order property" $(2012,581)$.

Because of this, Lyon's account fails to capture the features that make MEPPs different from ordinary program explanations. He may reply that the difference is that MEPPs work because they appeal to mathematical properties, but it is not obvious that mathematical properties can play this programming role. As Saatsi has pointed out, programming properties have an intuitively clear relationship with their corresponding lower-level causally-efficacious properties. It is hard to see how mathematics can enter in this relationship without a metaphysical account of the relation between mathematics and the physical world. "The programming relation", says Saatsi, "is a modal relation between properties" $(2012,582)$. Programming properties necessitate the instantiation

of a causally efficacious property, but it is not clear how a mathematical property would necessitate a physical instance. ${ }^{56}$

\footnotetext{
${ }^{56}$ Pincock (2015) addresses this issue (see $§ 30$ below).
} 


\section{§29. Marc Lange: MEPPs and mathematical necessities}

\subsection{Distinctive mathematical explanations}

Just as Lyon does, Marc Lange holds that MEPPs show that the explanandum was more necessary than could have been rendered by any of the causal processes that may have produced it.

If a fact has a distinctively mathematical explanation, then the modal strength of the connection between causes and effects is insufficient to account for that fact's inevitability $(2013,487)$.

According to Lange, MEPPs get their modal strength from pointing to relations of mathematical necessity in the physical world. As we saw in section $\$ 22$, for him, rather than describing "the world's network of causal relations" $(2013,509)$, MEPPs work by describing "the framework inhabited by any possible causal relation" $(2013,509)$. This is why MEPPs make an essential appeal to mathematics. By using modally stronger facts (i.e. mathematical facts), the MEPP is capable of pointing to these 'stronger necessities' in the physical world, and in this way, it gives us information that alternative causal explanations cannot.

These necessities are stronger than causal necessity, setting distinctively mathematical explanations apart from ordinary scientific explanations. Distinctively mathematical explanations in science work by appealing to facts (including, but not limited to, mathematical facts) that are modally stronger than ordinary causal laws - together with contingent conditions that are contextually understood to be constitutive of the arrangement or task at issue in the why question $(2013,491)$.

Now, for Lange a MEPP "exploits what the world is like as a matter of mathematical necessity" $(2013,496)$. In that sense, his view of MEPPs is committed to the existence of mathematical relations of necessity in the physical world, so it belongs to what I have called the 'descriptivist' view of MEPPs. For example, one of the cases Lange discusses most thoroughly is why a mother cannot evenly divide 23 strawberries among her three children. The explanation, says Lange, is that 23 is not a multiple of 3 . This mathematical fact is more necessary than any contingent fact about strawberries and children. Although it is true that the explanation involves objects with causal powers (strawberries and children), this is not relevant to the explanation. No collection of 23 objects, not even pseudo processes (which lack causal powers), can be divided by three, and this is why the strawberries cannot be evenly divided between three children (cf. Lange 2013, 496).

This is also what happens in the bridges case. The idea is that the system of bridges has not been crossed because it cannot be crossed. This impossibility is stronger than the impossibility associated to each particular unsuccessful attempt. Any particular attempt to cross the bridges is a causal process that necessarily will prove to be unsuccessful. From the perspective of a causal explanation, the fact that all these attempts have failed would be a coincidence. The MEPP, on the contrary, shows that this is no coincidence: it was necessary for all these attempts to fail, since the 
trip is mathematically impossible. On Lange's account, an Eulerian path over the bridges is mathematically impossible, and this is why it is physically impossible.

In the cicada case, what requires a mathematical explanation is not that cicadas with prime cycles have been 'chosen' by natural selection, as opposed to those with non-prime cycles. "Selection for", Lange points out following Elliott Sober, "is the causal concept par excellence" $(2013,499)$. Rather, what requires a mathematical explanation is the fact that, "in connection with predators having periodic life-cycles, cicadas with prime periods tend to suffer less from predation than cicadas with composite periods do" $(2013,499)$. The explanation shows that it is a matter of mathematical necessity that prime cycles have this property of 'minimizing intersection' with other cycles.

One interesting aspect of this account is that it helps to make sense of why MEPPs are noncausal explanations. As we saw in section $\$ 22$, although MEPPs are empirical applications of mathematics, this in itself does not render MEPPs causal, because they do not work by 'exploiting' these causal powers. Lange emphasizes that the why question answered by the MEPP presupposes that some facts in the explanandum are fixed. These are, as he puts it, the 'contingent conditions that are contextually understood to be constitutive of the arrangement or task at issue in the why question'. What needs to be explained, then, are some aspects of the structure of the physical system once it has been fixed by the why question $(2013,506)$. For example, with respect to the bridges case Lange says that:

[t]he bridges arrangement does not function in connection with the distinctively mathematical explanation as an initial condition that happens to persist (partly by virtue of various causal laws) during all attempts to cross the bridges. Rather, the why question itself takes the arrangement as remaining unchanged over the course of any eligible attempt $(2013,497)$.

Although the MEPP presupposes the causal powers of the objects involved (for instance, that "bridges are not brought into existence or caused to disappear by people travelling over the bridges" $(2013,497))$, this does not mean that the explanation works in virtue of those powers. For this reason, this is a noncausal explanation.

Now, Pincock has objected that Lange's view trivializes the discussion of the distinctive features of MEPPs. After all, Pincock argues, if even contingent facts can be fixed, then many physical phenomena would have a mathematical explanation (cf. Pincock 2015, 875). I believe, however, that Lange should not be worried by this charge of triviality. That there would be a MEPP for many simple physical facts, once they have been suitably defined, should not be taken as a weakness of the view. Perhaps some MEPPs are more interesting than others, but this in principle should not be a problem. There are certainly some ordinary scientific explanations that are more interesting than others! It would be a weakness of an account of scientific explanation if it cannot accommodate simple cases. 


\subsection{Problems with Lange's view}

There are, I think, two problems with Lange's account. First, he does not provide details about the source of the explanatory power of the explanation. And secondly, the sense in which MEPPs would appeal to facts that are mathematically necessary is not clear. With respect to the first problem, Lange says that MEPPs show that the outcome was necessary. But saying that something is necessary is not enough to explain it. Consider again the graph that represents the bridges of Königsberg. It is necessary that there will be a fixed number of paths available over the graph, none of them being Eulerian. This would be true in every possible world. As we saw, however, the brute computation strategy can be used to verify the truth of this claim, but it does not explain it. The crucial point is that the brute computation strategy also depends on facts that are more than physically necessary. So here we have a mathematical derivation that appeals to facts that are more than physically necessary, but does not provide explanatory information of the physical facts it represents. Unfortunately, Lange does not give details about how to differentiate this mathematical derivation and explanatory derivations such as Euler's proofs.

A second problem is Lange's commitment to the view that there may be 'mathematical necessities' in the physical world. This seems like a strong ontological commitment, so let us examine it more closely from the perspective of our two main examples. Let us start with the cicada case. The explanation that appeals to mathematical primeness in the cicada case would highlight the fact that it is necessary that prime-numbered cycles minimize intersection with other cycles. This would be true in any world where the idea of cycles makes sense, so this necessity would be stronger than physical necessity. Does this, by itself, support the claim that these relations are of mathematical necessity? Recall that this case depends on the simple notions of combination and equality. I do not believe combination and equality are inherently mathematical notions, but I do agree that in every world where physical lengths can be described by these two notions, the property of overlapping minimization would make sense. This fact may be modally stronger than other physical facts; but I do not believe there is anything to be gained by calling it mathematical. This point is perhaps clearer in the bridges of Königsberg case. The explanation relies on the extremely simple fact that every time one crosses a bridge, two pieces of land are involved, the starting point and the ending point. This fact seems to be modally stronger than other physical facts, and it is in virtue of this modally stronger fact that the explanation gains its explanatory force. But I do not think anything is to be gained by calling it mathematically necessary (more on this on section §38).

I have not argued that there are no mathematical necessities in the world; only that MEPPs do not presuppose the existence of these relations of mathematical necessity. We shall see in the next chapter whether there are other reasons to believe that the physical world actually has these features. For now, my point is that all we need for a MEPP is that the problem itself must be stated in a very precise way so that it fits the mathematical representation (for example, it must be very precise what we mean by 'bridge'). In order to do that, we must refine our description of the physical system so that we can build in the conceptual definition. Once we have this, mathematics will help us make explicit some of the ideas implicit in our conceptual definition of the problem at hand. 


\section{$\S 30$. Christopher Pincock: MEPPs and mathematical structures}

\subsection{MEPPs as abstract explanations}

In a recent paper (2015), Christopher Pincock presents an account of abstract explanations that analyzes the role of mathematics in MEPPs, which is an extension of Woodward's counterfactual account of scientific explanation. Recall that for Woodward fundamental in scientific explanations are objective relations of dependence between properties or events (see §9). These dependence relations are called invariances, and can potentially be used for manipulation and control. According to Pincock, causal dependence is not the only objective dependence relation that can be used in scientific explanations. Another dependence relation is the instantiation relation (2015, 865), and although he does not explicitly define this kind of relationship, he mentions two main features: i) if $a$ is an instance of $A$ then $A$ is more abstract than $a$ (abstract character); ii) if $a$ is an instance of $A$ then $A$ cannot be an instance of a (asymmetry). ${ }^{57}$ One example of the instantiation relation is the one between types and tokens. A type is more abstract than one of its tokens; a token is an instance of a type, but the type cannot be an instance of the token. These abstract properties, although having physical instances, are not themselves physical (cf. 2015, 865). For that reason, the instantiation relation is not causal; for Pincock, it does not make sense to talk of intervening over the abstract property in order to manipulate the instance.

With this view of instantiation in mind, Pincock defines abstract explanations as those where one explains features of the instances by appealing to features of the more abstract property. These explanations are ontic, because they depend upon objective relations of dependence; however, they are not causal, because, as we have seen, this dependence relation is not causal. Now, for Pincock "many mathematical structures have concrete systems as instances" $(2015,865)$. For that reason, MEPPs are a subclass of abstract explanations. Specifically, MEPPs are abstract explanations in which the instantiation relation is between a mathematical property and a physical instance. On this view, since mathematical structures have physical instances, the role of mathematics is not merely representational; it is to describe these mathematical structures in the physical world. In that sense, this is a descriptive account of MEPPs.

Now, we have seen that explanations that distinguish between levels of abstraction are not uncommon in science. Program explanations ( $\$ 8$ and $\S 28)$, for example, also appeal to more abstract properties in order to explain the explanandum, and as we saw, program explanations are all over the place in science. However, for Pincock there is a difference between program explanations and his abstract explanations:

[A]bstract explanations invoke a more abstract entity and its properties. Program explanations appeal only to a more abstract property of the physical system itself (2015, 873).

\footnotetext{
${ }^{57}$ James Cargile (personal conversation) has pointed out that this characterization leaves aside cases such as 'the property of being a property', which is not more abstract than the properties that instantiate it.
} 
In other words, program explanations appeal to higher-order physical properties, whereas abstract explanations appeal to non-physical abstract (and in some cases mathematical) properties.

Using this account, Pincock explicates the bridges case as follows. The structure of the bridges is an instantiation of the structure of a non-Eulerian graph. It is for this reason that we can explain facts about the physical system of bridges by appealing to facts about graphs. In particular, the impossibility of performing an Eulerian trip belongs both to the abstract graph and to its physical instance: ${ }^{58}$

Euler added his mathematical theorem that there is no return path through a graph that crosses each edge exactly once when at least one vertex has an odd valence. This is why there is no such return path across the bridges of Konigsberg. (2012, 206; my emphasis). ${ }^{59}$

For Pincock the graph that represents the bridges (a mathematical object) is not Eulerian, and this is why the bridges (physical things) cannot be crossed. In his (2015) paper, he does not explicitly address the cicada case, but we can understand it as an abstract explanation like this: because the life cycles instantiate the property of primeness, we can appeal to facts about the latter to explain the former. For example, 17 minimizes intersection with other numbers; this is why the life cycles minimize intersection with other life cycles.

Crucial to understanding this account is the notion of instantiation. In his (2012) Pincock explains what he means by a physical structure 'instantiating' an abstract structure:

Suppose we have a concrete system along with a specification of the relevant physical properties. This specification fixes the associated structure. Following Suarez, we can say that the system instantiates that structure, relative to that specification, and allow that structural relations are preserved by this instantiation relation $(2012,29)$.

I believe that what Pincock is saying here is that one discovers whether or not a system instantiates a mathematical structure depending on the level at which the system is described. Once this level of description is specified, it is a fact of the matter that the system instantiates the relevant mathematical structure, and so it possesses the properties associated with such mathematical structure.

\subsection{Problems with Pincock's view}

One problem with Pincock's view is that he does not provide details with respect to how exactly these explanations are carried out. Just as Woodward does, Pincock says that objective relations of dependence are necessary for explanation. But whereas Woodward is clear about how to use these dependencies in explaining (we must use them to perform interventions), Pincock is silent

\footnotetext{
${ }^{58}$ It seems that for Pincock the impossibility belongs to the two structures in the same sense, because the physical system possesses the mathematical property of being an Eulerian graph.

${ }^{59}$ Note that Pincock is addressing the problem of whether an Eulerian circuit is possible. This is not the problem Euler aimed to solve, although for philosophical purposes this distinction is unimportant.
} 
about this issue. Pincock rejects the possibility of performing 'interventions', so for him a physical property gets explained just by showing it to be an instance of a mathematical property. In fact, he understands his view as providing support of Baker's rejection of Steiner's view $(2015,847$, $\mathrm{fn} 12$ ). Because of this, Pincock's account is open to the same criticism I made of Baker's (and Lange's) account. Even if there are mathematical structures instantiated in the physical world, in order to explain physical features it would not be enough to show that truths about mathematical structures apply to the physical instance; an explanation must show how the explanandum depends on some properties of the structure. Without this information, we do not have an explanation.

Another problem of Pincock's view is its reliance on the notion of physical structures as instantiations of mathematical structures, as opposed to defining them as nominalistic structures capable of being represented by mathematics. Pincock argues against this possibility saying that this representational strategy does not work for mathematical explanations. For him, if mathematics were simply a representational or indexical device, it would be incapable of isolating explanatorily relevant features of a phenomenon (cf. 2012, 208-10). For Pincock, explanations where recurring features are isolated are noncausal explanations precisely because they fail to fit the indexing or representational approach.

Indexing here is a technical term, used amongst others by van Fraassen (e.g. 2008), that refers to the representational role of data models and their nonmathematical target systems. A data model is the first level of mathematical models that is constructed with data sets obtained by measurement procedures (see Suppes 1962 for a discussion of data models, and van Fraassen 2008 for a detailed account of the indexical role of mathematics). Pincock's idea is that if the role of mathematics in applications is ultimately indexical, the physical world in itself does not have mathematical features, and therefore it would be hard to explain how a mathematical structure can be representational at all:

Indeed, it is hard to see how the indexing approach is even supposed to get off the ground when the mathematics is isolating recurring features... we typically lack the knowledge to characterize these features in non-mathematical terms. But even if we could, a physical characterization of those features risks introducing irrelevant aspects of the systems in question $(2012,210)$.

Pincock's point is that a mathematical representation is not going to necessarily be restricted to the point where it represents only what is explanatorily relevant. Many cases would have "surplus" mathematical structure that is not relevant for the explanation. This is why, for Pincock, we must introduce the notion of instantiation.

I believe, however, that this charge against the indexical or representational approach is unjustified, because there is no risk of introducing irrelevant aspects in the explanation. The choice for an appropriate mathematical representation is made considering the background knowledge about what is and what is not relevant to the explanation; this is done prior to the choice of mathematical structure to be used in the explanation. 
I have already discussed the problems with this notion of instantiation in section $\S 13$, but let me come back to it to see how it works in relation to Pincock's criticism of the indexing or representational approach. Let us examine once again Mauricio Suárez's quote mentioned in section $\S 13$ (cited and subscribed to by Pincock (cf. 2012, 29)). For Suárez, the notion of instantiation must be understood as follows:

We may say that an object instantiates a structure if there is some division in parts and relations of the object that agrees with the structure [...] Since there are always different ways of cutting out its domain of elements and relations, every physical object instantiates simultaneously several structures. The physical world underdetermines its mathematical structure - which may only be ascribed under a particular description $(2010,96)$

For Suárez, a physical system can 'instantiate' many mathematical structures. Because of this, there is always the question of how do we choose, among these structures, the one that is relevant for a given scientific purpose. The idea is that whether or not a physical system instantiates a mathematical structure depends on specific theoretical assumptions about such a system. In a scientific explanation, these are assumptions about the features that are relevant to explaining the explanandum. Pincock argues that if these features were not somehow mathematical, the mathematical apparatus would not be capable of highlighting the relevant explanatory physical features. But as we have seen, the very process of instantiation consists in highlighting the features that can be represented with a specific mathematical structure. This is precisely what underlies the process that I have called 'optimal representation'. In my account, we want to understand why $P$ has property $\mathrm{P}^{*}$, and we represent $\mathrm{P}$ with the mathematical model $\mathrm{M}$. The crucial point is that the fact that we chose a particular model $\mathrm{M}$, rather than, say, model $\mathrm{M}^{\prime}$, depends on our background knowledge, that is, on our previous understanding of what is relevant to the explanation. And it is only after the representation is in place - a representation that is loaded with our own theoretical assumptions about what is relevant to the explanation that we can provide a mathematical explanation. Given what we know to be relevant in a given situation, we decide to represent the physical system as instantiating (in Suárez's sense) a given mathematical structure and we interpret this structure in empirical terms.

The inferential conception of the applicability of mathematics addresses this issue without appealing to the notion of instantiation. The question is, how do we choose the right mathematical structure to represent a physical phenomenon in a given situation. Bueno \& Colyvan point out that:

[e]ven if there is some privileged way of carving up the world into objects and relations (and, of course, it is extremely controversial that the world co-operates in this way, providing natural joints, as it were), such a carving, it would seem, is delivered by our theories, not by the world itself $(2011,347)$.

The idea is that, by choosing one mathematical model (suitably interpreted) rather than another, we assume that the physical system has certain structural features. The role of the mathematical model is to represent these features. But in order to do this, we do not need the notion of instantiation. Let us see how this works in our cases. 
In the hand shaking case of section $\S 21$, for example, even though we may not know why there have been 45 hand-shakings between 10 people, we know that the only relevant things are the connections between people, and we know that things like height, hair color, etc. are irrelevant. And it is with this background knowledge that we decide to represent these features using a complete graph. The system of 10 people can be represented by many different mathematical structures, depending on how we describe it, but given our background knowledge about what is explanatorily relevant in this particular explanatory context, we choose to represent it with a complete graph.

This is even more evident in the bridges case. Recall Elher's letter to Euler, where he suggested that the solution to the problem may require Leibniz's Calculi Situs (§18). Elher already knew that distances and such were not relevant, and Euler mentioned the same idea to Marinoni in his letter. Before providing a solution, before even choosing a particular mathematical model, it was evident for these two men which things were relevant and which things were not. The choice of a mathematical graph is an expression of this background knowledge. This graph does not explain why such a path is impossible; rather, by working over the graph Euler showed how the relevant physical facts do so. It tells us that areas, distances, etc. are not relevant, but connections are.

Likewise, in the cicada case, it was their background knowledge about the importance of natural cycles in explaining animal behavior that led scientists to take years as the relevant measurement unit, and to idealize years as equal to each other. Once this model is in place, we can forget about the cicadas and focus on the prime number of years, and so we have a mathematical fact, which is that, say, 17 minimizes intersection with numbers less than 34 . One can use time-lengths to illustrate this principle. But the fact that these time-lengths exemplify this mathematical theorem does not mean that they are explained by it. Again, the mathematical representation show us how the real explanatory facts about time explain the life cycles, but those facts about time are not explained by the mathematical representation.

From my discussion, what is important to note is that theoretical background knowledge about relevant features is an essential part of the process of choosing the right mathematical model to be used in a MEPP. It is because of this, I have argued, that the representationalist approach cannot be easily dismissed to explicate what is going on in these cases. But of course, there may be other reasons to adopt the realist approach (what I called the 'descriptive' view at the beginning of this chapter). I analyze (and reject) what is considered to be one of the main arguments for the descriptivist approach in the next chapter.

\section{$\S 31$. Conclusion}

The descriptive accounts I have discussed in this chapter appeal to the existence of physical properties in the physical world. As I have shown, however, this assumption is not needed in order to explicate what is special about MEPPs. But perhaps there are, after all, mathematical properties in the physical world. This lead us to the Enhanced Indispensability Argument. 


\section{CHAPTER 5. The Indispensability Argument}

\section{$\S 32$. Introduction}

The Indispensability Argument (IA) relies on the applicability of mathematics in science to support mathematical realism. The explanatory version of the IA focuses on the indispensability of mathematics in scientific explanations, and relies on the principle of Inference to the Best Explanation (IBE) to justify its conclusion. The idea of this explanatory IA is that if we believe in the existence of the concrete unobservable posits that feature in our best scientific explanations, we should also believe in the mathematical posits of those explanations. However, against the explanatory IA it has been objected that the role of mathematical posits in scientific explanations is to represent concrete explanatory facts and help draw inferences about those facts, but that mathematical posits, by themselves, are not explanatory. This has been taken as a weakness of the explanatory $I A$, and so a new version of the argument has been recently advanced, known as the 'Enhanced Indispensability Argument' (EIA). The EIA relies on the fact that there are mathematical explanations of physical phenomena (MEPPs) to support the claim that mathematics can play an explanatory role beyond its representational and inferential roles. According to the EIA, if we apply the IBE principle to MEPPs, mathematical realism would be justified. In this chapter I use my account of MEPPs to refute the EIA.

I start by introducing the original IA (§33), and discuss criticisms by Charles Parsons, Elliott Sober and Penelope Maddy (§34). Next, I present the explanatory IA (§35), and, after presenting criticisms from Hartry Field and Joseph Melia, I discuss the motivation for the Enhanced Indispensability Argument (EIA) (\$36). I then use the account of MEPPs that I have developed in the previous two chapters to show that the EIA fails. First, I show that in order for mathematics to be indispensable in explanations in a way that carries ontological commitments, the explanandum must already be committed to the existence of mathematical properties and entities, which renders the EIA circular (§37). Next, I show that the indispensability of the mathematical component in MEPPs is pragmatic, and that, ultimately the role of mathematics is representational and inferential (\$38). For this reason, the existence of MEPPs does not have the ontological consequences required by the EIA. My goal is not to directly defend mathematical nominalism, but to show that the existence of MEPPs does not support mathematical realism.

\section{$\S 33$. Quine's indispensability argument}

The original indispensability argument is based on W.O. Quine's doctrines of naturalism and confirmational holism. According to Quine, naturalism is:

[The] abandonment of the goal of a first philosophy. It sees natural science as an inquiry into reality, fallible and corrigible but not answerable to any supra-scientific tribunal, and not in need of any justification beyond observation and the hypothetico-deductive method (cited in Resnik 2005, 416).

This is what Michael Resnik calls 'ontological naturalism', the view that science is the ultimate arbiter of existence. For Quine, ontological naturalism implies that "it is within science itself, and 
not in some prior philosophy, that reality is to be identified and described" (cited in Resnik 2005, 427). The idea is that, because of its success, science is the only enterprise that gives us an accurate picture of the world. It is this naturalistic premise that entitles us to believe in the existence of the entities or processes postulated by our best scientific theories, being observable like rocks and apples, or unobservable like genes, electrons, radio waves and electromagnetic fields.

Quine also defended the thesis of confirmational holism, which is that claims of theoretical science are not confirmed or refuted in isolation, but only as a part of a system of hypotheses. Scientific hypotheses are never tested alone, but in conjunction with auxiliary hypotheses:

The totality of our so-called knowledge or beliefs, from the most casual matters of geography and history to the profoundest laws of atomic physics or even of pure mathematics and logic, is a man-made fabric which impinges on experience only along the edges (cited in Resnik 2005, 414; my emphasis).

On this view, if empirical evidence confirms a part of a theory, the whole theory gets confirmed, including its mathematical components. Since mathematics implies quantification over numbers, functions, sets, etc., the belief in the existence of these mathematical entities is justified. In Quine's words:

Ordinary interpreted scientific discourse is as irredeemably committed to abstract objects - to nations, species, numbers, functions, sets - as it is to apples and other bodies. All these things figure as values of the variables in our overall system of the world. The numbers and functions contribute just as genuinely to physical theory as do hypothetical particles (cited in Colyvan 2001, 10).

This has been called the 'Indispensability Argument' (IA). The basic idea is that, because of naturalism, we should be committed to the truth of the statements that are indispensable to our best scientific theories; and because of holism, we should be committed to all the objects and processes over which those statements quantify. Since quantification over mathematical objects is indispensable to the success of these theories, we ought to be committed to the existence of the mathematical objects mentioned in those statements. Mark Colyvan schematizes the argument as follows:

P1: We ought to have ontological commitment to all and only those entities that are indispensable to our best scientific theories;

P2: Mathematical entities are indispensable to our best scientific theories.

C. We ought to have ontological commitment to mathematical entities (Colyvan 2001, 11).

The importance of the IA has been acknowledged by supporters and detractors of mathematical realism. Mark Colyvan (2001) takes it to be responsible for one of the most dominant debates in the philosophy of mathematics, and Hartry Field thinks it is "the only nonquestion-begging" argument for mathematical realism (1980, 4). 
Now, with respect to the nature of the mathematical objects whose existence may be supported by the IA, Colyvan says the following:

[The IA] simply asserts that there are mathematical objects... any (realist) account of mathematical objects is all right by the indispensability argument. It must, of course, be consistent with the view that mathematics has an empirical character (which is the only real restriction) (2001, 142).

This notion of empirical character is explicated by Quine in later formulations of the argument, where he points out that:

[i]nsofar as mathematics gets applied in natural sciences, I see it as sharing empirical content. Sentences of pure arithmetic and differential calculus contribute indispensably to the critical semantic mass of various clusters of scientific hypothesis and so partake of the empirical content imbibed from the implied observation categoricals (1991, 269).

The notion of observation categoricals provides a softer version of confirmational holism. Back in Two Dogmas, the unit of 'empirical significance' was 'the whole of science', but later Quine formulated it in terms of 'clusters of sentences just inclusive enough to have critical semantic mass (i.e. are open to confirmation). An observation categorical, then, is a generalization that conditionally connects two observation sentences (e.g. 'whenever this, that'). The empirical character of mathematics implies that, since mathematics is applied in the sciences, and scientific claims have critical semantic mass, mathematical statements are open to empirical refutation.

As we have seen at the beginning of the previous chapter (§25), descriptive accounts of MEPPs assume some hybridity between the physical and the mathematical realms, and allow the possibility of mathematics being revised in the face of experience. These are accounts that endorse the 'empirical character' of mathematics. Colyvan's point is that, apart from the fact that mathematics must be somehow empirical (which rules out pure mathematical Platonism), the question about what kind of objects are these mathematical objects is not settled by the IA.

The most discussed criticisms against the IA are those of Charles Parsons, Elliott Sober, Penelope Maddy, Hartry Field, and lately Joseph Melia. Parsons and Sober object that mathematics is unfalsifiable, and so it should not inherit the confirmation of the scientific theories that use them. In turn, Maddy objects that naturalism (as understood by Quine) is not compatible with confirmational holism. As we will see, this poses a problem when mathematics quantifies over idealized concrete posits. On the other hand, Field targets the second premise of the IA. He argues that, in principle, mathematics is dispensable from our best scientific theories. In turn, Melia argues that the role mathematics plays in science is not enough to justify the conclusion of the IA. Some of these objections have elicited reformulations of the IA. In the following sections I will present these objections, discuss some replies by Michael Resnik and Mark Colyvan, and then focus on the newer version of the IA. 


\section{§34. Problems with confirmational holism}

\subsection{The unfalsifiability of mathematics}

\section{a) Charles Parsons and Euclidean rescues}

One complaint against the IA has been that mathematics seems to be unfalsifiable. Charles Parsons, for example, has pointed out that if mathematical theories inherit a share of the confirmation of scientific theories, one would expect them to be on a par with regard to falsification as well. But this does not seem to be true of mathematics:

[N]o proposition of pure mathematics has been falsified. [For example,] no proposition of Euclidean geometry is falsified by the discovery that physical space is not Euclidean (cited in Colyvan 2001, 125).

Resnik replies to Parson using what he calls 'Euclidean rescues'. For Resnik, although it is true that Euclidean geometry remains unfalsified as an abstract theory of Euclidean spaces, it was indeed falsified as a theory of physical space. These Euclidean rescues, Resnik argues, can in principle be applied to any falsified theory. The idea is that in cases of falsification, the mathematical parts of a theory should be interpreted as highlighting an abstract structure, rather than a concrete structure (Busch 2012, 500). I do not see, however, how this answer helps the mathematical realist. If it is true that even false theories can be rescued, why would science be important to justify Platonism, as the IA defendant holds? If empirical falsifications do not affect the status of a mathematical theory, then whether or not that theory is conceived as quantifying over fictional or real posits is independent of its application in science (as I will show below, this is also why Resnik's pragmatic IA fails).

\section{b) Elliott Sober and the Maxim of minimal mutilation}

Another criticism to the IA has been pointed out by Elliott Sober. According to him, it is very common that different scientific hypotheses share the same mathematical corpus in their assumptions, and so those mathematical assumptions are never tested. For mathematics to be actually tested, there must be mathematics-free competitors, or at least alternative mathematical theories as competitors. But this would make the IA implausible:

[D]o we really have alternative hypotheses to the hypotheses of arithmetic? If we could make sense of such alternatives, could they be said to confer probabilities on observations that differ from the probabilities entailed by the propositions of arithmetic themselves? I suggest that both these questions deserve negative answers (cited in Colyvan 2001, 128).

Now, a distinction between confirmation and falsification can be established using Quine's 'maxim of minimal mutilation', which is an application of Occam's razor ("when choice is otherwise undetermined, opt for economy" (Quine 1991, 269)). When a cluster of sentences with critical semantic mass gets confirmed by empirical observations, all of the sentences get confirmed; but when it gets disconfirmed, it is rarely the fault of every member of the cluster. One false 
assumption is enough to render the whole set of claims false. So how to choose? The maxim of minimal mutilation dictates that when revising a set of claims, we must try to avoid altering beliefs that occupy a central place in our web of beliefs, because revising these central beliefs would have ramifications in many other areas of the web. As Quine points out, this is precisely the case of our mathematical beliefs:

$[W]$ e are disinclined to tamper with logic or mathematics when a failure of prediction shows there is something wrong with our system of the world. We prefer to seek an adequate revision of some more secluded corner of science, where the change would not reverberate so widely through the system (cited in Colyvan 2001, 126).

Colyvan argues that this maxim of minimal mutilation can be used to reply to Sober's objection. The fact that many theories share mathematical assumptions does not mean that these assumptions are untestable, it only means that these assumptions are useful in many different contexts. In fact, this maxim is applied in a variety of other contexts. As Colyvan points out:

Hypotheses about electrons (notoriously) have been employed by many false theories, and yet we are unwilling to blame them for the lack of empirical support for the theories in question. Astrologers refer to the orbits of the planets in grossly false theories about human behaviour, and yet we are not about to blame the planets for the lack of empirical support for astrology $(2001,132)$.

The maxim of minimal mutilation dictates that, in the face of falsification, we should not start by questioning the assumptions that are central to many different theories. This does not mean, however, that those shared assumptions are untestable.

Now, to be fair to Sober, all that Colyvan's reply shows is that the fact that mathematics is shared by many branches of science does not render it untestable in principle. But neither Colyvan nor Quine nor Resnik have presented a positive case for the testability of mathematics: why would mathematical theories, which quantify over abstract mathematical objects, be abandoned as such simply because one assigned physical interpretation has turned out to be incorrect? To my mind, these authors have not advanced a convincing case to support this. As we will see, this is not the only problem with confirmational holism.

\subsection{The incompatibility of naturalism and confirmational holism}

Penelope Maddy advances two criticisms of the IA. First, she questions the compatibility between naturalism and confirmational holism. According to her, we ought not to have an ontological commitment to all the entities indispensable to our best scientific theories $(2005,454)$. Maddy does not reject the naturalistic assumption that only science tells us what exists and what is true; her point is that not all the entities indispensable to science exist. Note that this first criticism applies to scientific theories in general, and not only to mathematical posits. That is, Maddy points to problems of reconciling naturalism and confirmational holism in general, not necessarily related to indispensability arguments. Consider the following examples: in fluid dynamics, matter is assumed to be continuous, but scientists do not take matter to be continuous; for explaining 
waves, the ocean is represented as infinitely deep, but nobody thinks this is actually the case; in classical mechanics, some kinematic properties are calculated by assuming frictionless planes, but no such things exist in the world. As Maddy points out:

If we can see, in the course of our scientific study of science, that certain parts of our theory... don't reflect what's actually present in the physical situation... then it seems reasonable to conclude that these parts of our theory are not, in fact, confirmed by our scientific methods $(2005,456)$.

The idea of this criticism is that by naturalism, we should respect the methods of working scientists, and working scientists often appeal to entities and processes that they do not take to be true; but according to confirmational holism, theories get confirmed as wholes. So there seems to be an incompatibility between these two principles, and Maddy rejects confirmational holism. The question now is on what side should we put mathematics once we abandon the picture of scientific theories as homogeneous units. According to Maddy, if mathematics is used to quantify over idealized physical objects (such as frictionless planes, infinitely deep oceans, etc.), then it should not receive a share in confirmation when the theory gets confirmed, for the same reason that beliefs in those idealized physical objects do not get confirmed either. This is also true for Quine's moderate holism, according to which it is clusters of statements, rather than whole theories, that get confirmed as wholes. Maddy's point is that, when those clusters include idealizations, those idealizations are never confirmed when the cluster gets confirmed.

In his reply to Maddy, Colyvan points out that the indispensability argument is about those entities that are indispensable to science. Idealizations such as frictionless planes, says Colyvan, are not indispensable (the same can be said about infinitely deep oceans and the continuity of matter). They are introduced for pragmatic purposes only:

Appeal to frictionless planes simply makes the statement of certain laws of mechanics easier, so omitting such appeals makes little difference to the overall theory... [In that sense,] frictionless planes are dispensable to the theory of mechanics (Colyvan 2001, 99 fn11).

I believe that Colyvan is correct in the case of Galilean idealizations, such as frictionless planes introduced for tractability purposes, or in the case of minimal model idealizations that can be cashed out in empirical terms, such as some explanations of the bridges of Königsberg case. But Maddy's point still stands, because there are other kinds of mathematical models where not all false assumptions are dispensable in the way Colyvan suggests. As we saw in sections $\S 12$ and $\S 15$, non-deidealizable idealizations are also part of scientific practice, like asymptotic explanations. In those cases, some of the mathematics used quantifies over false objects in a way that is indispensable to the explanation, but, again, we are not committed to the existence of these objects. 


\subsection{Resnik's pragmatic IA}

Maddy's argument relies on a distinction between parts of a theory that are true, and parts that are merely useful:

If we remain true to our naturalist principles we must allow a distinction to be drawn between parts of a theory that are true and parts that are merely useful. We must even allow that the merely useful parts might in fact be indispensable, in the sense that no equally good theory of the same phenomena does without them. Granting all this, the indispensability of mathematics in well-confirmed scientific theories no longer serves to establish its truth (cited in Resnik 1997, 46).

Now, Resnik argues that even if we grant this distinction, the IA would stand. That is because, for him, it is the mere usefulness of mathematics in science that grants commitment to mathematical posits. Resnik has pointed out that when scientists use mathematics, they presuppose the truth of these mathematical theories, even in cases where they know their physical theories to be false:

[A] careful analysis of the way scientists use mathematics reveals that they presuppose its truth. Even when using such devices as point-masses, frictionless objects, or ideal gases to develop idealized models, they presuppose the reality of the mathematical objects to which they refer $(1997,6)$.

Resnik acknowledges that this leads to a different version of the IA, one that does not rely on confirmational holism. He calls it the 'pragmatic' indispensability argument $(1997,46)$. The basic idea is that even when quantifying over false objects, science needs mathematics. Resnik thinks that these applications of mathematics would not be possible if mathematics were false. On this view, successful theories confirm their derivation methods. Here is Resnik's pragmatic IA in standard form:

P1p. In stating its laws and conducting its derivations science assumes the existence of many mathematical objects and the truth of much mathematics

P2p. These assumptions are indispensable to the pursuit of science; moreover, many of the important conclusions drawn from and within science could not be drawn without taking mathematical claims to be true.

$C_{p}$. So we are justified in drawing conclusions from and within science only if we are justified in taking the mathematics used in science to be true (Resnik 1997, 46-7)

Resnik notes that this version of the IA does not presuppose that our best scientific theories are true. It applies whenever scientists presuppose the truth of mathematics, even in theories that have been empirically falsified (e.g. Newtonian physics). What is more, the argument does not presuppose that the evidence for science is also the evidence for mathematics (cf. 1997, 47). In that sense, the justification for mathematical realism is not the same as the one for scientific 
realism. One can be a scientific antirealist and still believe in the truth of mathematics (cf. 1997, 6 ). It is the indispensability of mathematics in doing science that justifies its truth, regardless of whether it is confirmed science or not.

It seems to me, however, that this version of the IA faces a serious problem: the argument presupposes the truth of mathematics before it is successfully applied in science. Resnik's pragmatic IA requires us to have "quite a bit of independent evidence from mathematics" (1997, 47), which comes from our everyday practices of measuring, counting, etc. (cf. 1997, 48). But if this is all that is needed to justify mathematics, then we do not need the IA as support for its truth. If mathematics can be justified by false scientific theories just because they presuppose the correctness of mathematical derivations, why would it be necessary to appeal to science in the first place? Evidently, purely mathematical practice also requires mathematical derivations, but we do not take mathematics itself to be justified by it, at least not when our main purpose is to argue for the truth of mathematics by appealing to its applicability in science. But what would be the gain of successful applicability, if unsuccessful applicability also justifies the truth of these theories? Resnik's modification of the IA renders it circular.

\section{§35. The explanatory indispensability argument}

\subsection{Inference to the best explanation}

One special kind of indispensability argument, the one most discussed in the literature ${ }^{60}$, focuses on indispensability for explanations. This version of the IA is based on the principle of Inference to the Best Explanation (IBE), which is explicated by Hartry Field as follows:

[S] uppose (a) that we have certain beliefs, beliefs about 'the phenomena', which we are unwilling to give up; (b) that this class of 'phenomena' that we believe in is large and complex; (c) that we have a pretty good explanation of these phenomena (in the sense of, a relatively simple non-ad hoc body of principles from which they follow); and (d) one of the assumptions that appears in this explanation is claim $S$, and we are pretty sure that no explanation of the phenomena that does without claim $S$ is possible. The idea of 'inference to the best explanation' is that under these circumstances we have a strong reason to believe claim $\mathrm{S}(1989,15)$

The IBE principle entails that if a belief is indispensable to the best explanation of a given phenomenon, then we should believe it, "regardless of whether that belief is itself observational, and regardless of whether the entities it is about are observable [entities]" (Field 1989, 15). Field points out that one reason why the difference between observable, unobserved, and unobservable is unimportant for the application of IBE is that every time we rely on the IBE principle we are believing something beyond what we have observed:

\footnotetext{
${ }^{60}$ This version of the IA was widely discussed in the literature years before Baker (2005) and Colyvan (2001) presented the Enhanced Indispensability Argument (see e.g. Field 1989; Steiner 1978b). The EIA relies on so called genuine MEPPs.
} 
The fact that one belief could be fairly directly tested by observation while the other couldn't seems to have no relevance to their evidential status when such an independent test has not been made. (When the independent test has been made [...] then we need no longer rely on inference to the best explanation. When we do rely on inference to the best explanation, our beliefs go beyond the observations we have made, and my point is that the difference with respect to possible observations that haven't been made is irrelevant to our actual evidential situation.) $(1989,16)$

There are, however, two cases where the IBE principle does not apply. First, according to Field, the IBE principle does not apply to so called 'as-if' claims, that is, claims of the form "the phenomena are as they would be if explanation E were correct" $(1989,15)$. These claims do not start from accepting the truth of the explanandum; rather, they aim at establishing the truth of the explanandum because there is a nice candidate explanation in the offing. 'As-if' explanations cannot be used to establish ontological commitments based on the IBE principle, because they are more similar to arguments than to explanations: they assume the correctness of the explanation in order to establish truths about the explanandum (as I will show (§37), Baker made this mistake in his first reconstruction of the cicada case to defend his version of the IA). In addition, Joseph Melia has pointed out that not all the elements involved in an explanation are responsible for the explanatory power of the explanation, and only those that are should be interpreted realistically. These elements are said to be playing a genuine explanatory role, and the IBE strategy applies only to them. Some authors have pointed out that to play an explanatory role is to be indispensable to the explanation. But as Melia has pointed out, it is not enough for a posit to be indispensable to the explanation: it has to be indispensable in the right way.

\subsection{The explanatory IA}

The explanatory version of the IA substitutes the first premise of Quine's IA with the IBE principle, and appeals to an alleged indispensability of mathematics in scientific explanations to conclude that by IBE we should accept the truth of the mathematical statements that feature in these explanations, and consequentially, the existence of the mathematical objects posited by those statements. In standard form the argument would be as follows:

$\mathrm{P} 1_{\mathrm{E}}$ : We ought rationally to believe in the existence of any entity that is indispensable to our best scientific explanations.

P2 $2_{\mathrm{E}}$ : Mathematical objects are indispensable to our best scientific explanations.

C: Hence, we ought rationally to believe in the existence of mathematical objects (Adapted from Baker 2009, 613).

It is important to note that the argument does not simply make an analogy between explanations that require postulation of concrete unobservables and explanations that require postulation of mathematical entities. As Field points out, the argument is actually stronger. It says that the same scientific explanations that posit unobservables also posit mathematical entities. In that sense, it is not possible to distinguish between different kinds of explanations, labeling as 
weaker those that postulate mathematical entities, and stronger those that rely only on concrete objects (Field 1989, 17). This actually makes this version of the IA more pressing for scientific realists than for other philosophers. The argument claims that the same explanations that require us to believe in unobservable concrete objects or processes, also require us to believe in abstract mathematical objects. Of course not all philosophers are scientific realists, but the point of this version of the argument is, as Hilary Putnam has pointed out, that if you admit that theoretical entities exist, then you would be intellectually dishonest if you do not grant that mathematical entities exist as well (cf. Colyvan 2001, 10-11).

\subsection{Objections to the explanatory IA: The hard road and the easy road}

Most criticisms of this argument focus on the indispensability thesis (P2). According to Hartry Field, there is a fundamental difference between quantification over mathematical entities and theoretical entities. For Field:

Truth is not required for goodness... what is required instead is something call conservativeness, which involves some of the features of necessary truth without involving truth $(1989,4)$.

Mathematics is useful in science because mathematics is conservative over the non-mathematical world:

(C) CONSERVATIVENESS: A mathematical theory $M$ is conservative if and only if for any assertion $\mathrm{A}$ about the physical world and any body $\mathrm{N}$ of such assertions, A doesn't follow from $N+M$ unless it follows from $N$ alone $(1989,58)$.

For Field, this marks a fundamental difference between mathematics and concrete physical posits. If the nominalistic theory is consistent, then every claim about its domain will follow entirely from its nominalistic premises. Adding mathematics to a scientific theory does not carry any new consequence about the theory's empirical domain. The purpose of adding mathematics is to help find out about those consequences by shortening derivations within the nominalistic theory. On the contrary, the postulation of new concrete objects typically results in a theory that has new implications about its domain. In principle, Field argues, in science any inference from a set of premises to a set of conclusions that can be made with the help of mathematics can also be made without it; but this is not the case of theoretical entities. Because of this, Field says, scientific theories can in principle be reformulated without using mathematics, and in that sense mathematics is dispensable in a way theoretical entities are not.

Field's strategy has been called 'the hard road to nominalism' (cf. e.g. Colyvan 2010), since it refutes the indispensability of mathematics by showing how science can be reformulated in nominalistic terms (hence the title of Field's 1980 book: 'Science without Numbers'). This strategy has received many criticisms (see Bueno 2014 for a summary); but let me focus on the two that are relevant to my discussion. 
Though reluctant to accept mathematical realism, Joseph Melia argues that we should not attempt to eliminate quantification over abstract entities in science because this should imply going against current scientific practice $(2002,454)$, and it is very unlikely that scientists will change their practice of quantifying over abstracta. As he puts it, "if we really are to respect the practice of scientists, we ought to accept the abstract ontology which their practice commits them to" (2002, p.457). In addition, Melia claims, in practice it is impossible to express some scientific contents without mathematics, especially if we consider mathematics' great representational power.

Melia's strategy against the indispensability thesis is to respect the practice of using mathematics in science - which consists in quantifying over mathematical objects - while at the same time refraining ('weaseling away') from accepting these objects in our ontology (hence the title of Melia's 2000 article: 'Weaseling Away the Indispensability Argument'). This is in fact, according to Melia, what most scientists do in practice. But how do we know when to apply the 'weaseling' strategy? The idea is that mathematical entities and concrete unobservable entities do not play the same role in science. Although both concrete unobservable entities and mathematical entities may be indispensable to science, they are not indispensable in the same way. Quantification over concrete unobservables is ontologically committing, whereas quantification over mathematical entities is not; this is because, contrary to concrete unobservables, mathematical entities give us information about the world by playing an indexical or representational role, and an inferential role. Specifically, "mathematics can enable us to express possibilities about the concrete world that may not be expressible in nominalistically acceptable language." (2000, 255). Because of this, mathematics is indeed very useful for doing science, even indispensable in practice, but this does not mean that beliefs in mathematical entities are justified by this usefulness:

[M]athematics is the necessary scaffolding upon which the bridge [of science] must be built. But once the bridge has been built, the scaffolding can be removed (Melia 2000, 469).

Now, as we saw, the IBE principle entails that our commitments to concrete unobservables are grounded on the fact that some of our best scientific theories gain their explanatory power from positing the existence of those entities or processes (we believe in the existence of electrons, or in evolution, in part because of the vast array of phenomena that can be explained by positing them); but this is not the case of the role of mathematics. As we have seen $(\S 14, \S 15)$, mathematical statements feature in our best scientific theories because these mathematical statements can correctly represent some aspects of the physical world and help us draw inferences about concrete objects. In that sense, the role of mathematics in science is merely representational and inferential, as stated by the inferential conception. ${ }^{61}$ Even when mathematics features in scientific explanations, it does not play an explanatory role. This strategy has been called 'the easy road to nominalism' (cf. Colyvan 2010) since, contrary to Field's strategy, it does

${ }^{61}$ Representational is understood here a broad sense. Mathematics represents (highlights) physical features by directly representing them, or by isolating them by indexing what is and what is not relevant in a given context (see section $\S 12$ ). 
not require the elimination of mathematical vocabulary from scientific theories (see Melia 2000, 2002, and also Bueno 2012).

Melia's challenge for defendants of the explanatory indispensability argument is to show that mathematics can play an indispensable explanatory role in science, not only that it provides us with good representations of the relevant physical properties that feature in successful explanations. This has given rise to a modified version of the explanatory IA, which focuses not in scientific explanations in general, but in mathematical explanations of physical phenomena, where, allegedly, the role of mathematics is genuinely explanatory. Here is where the topic of this dissertation becomes relevant to this discussion in the philosophy of mathematics.

\section{$\S 36$. The Enhanced Indispensability Argument ${ }^{62}$}

Alan Baker (2005) and Mark Colyvan (2001) attempt to reformulate the explanatory IA in a way that accommodates Melia's challenge. The key to the success of their project is to call attention to the existence of purely mathematical explanations of physical phenomena (MEPP) in science. Because of this, on their view, mathematical realism would be justified if we reason like this:

P1*: We ought rationally to believe in the existence of any entity that plays an indispensable explanatory role in science.

P2*: Mathematical objects play an indispensable explanatory role in science.

C: Hence, we ought rationally to believe in the existence of mathematical objects (cf. Baker 2009, 613).

This has been called the Enhanced Indispensability Argument (EIA). In Colyvan's words, "if mathematics is contributing directly to explanations, it is hard to see how any scientific realist can accept the explanations yet deny the truth of the mathematics" (Colyvan 2007, 120).

This new version of the IA depends on whether some of our best scientific explanations indispensably depend on their mathematical part. In other words, the EIA relies on whether mathematics can be indeed indispensably explanatory in the sense required by premise P1*. It is for this reason that the analysis of MEPPs is important. If it is found that the role of mathematics in MEPPs is genuinely explanatory, then according to Baker, Colyvan and Melia, mathematical realism would be justified because, as Baker puts it, "the mathematical postulates would have virtues that the nominalist has already conceded carry ontological weight" (Baker 2005, 225). Melia claims that there are no such cases, but Baker and Colyvan claim that there are - precisely the MEPPs that I have discussed in previous chapters. In the following sections I will show that the EIA can be resisted by adequately distinguishing between the physical and the mathematical explanandum, and showing that the mathematized version is not superior, in the relevant respects, to the nominalized version (\$37). I will then use my own account of MEPP to argue that the EIA is unsound because the sense of indispensability of $\mathrm{P} 2^{*}$ is not the one required by $\mathrm{P}{ }^{*}$

\footnotetext{
${ }^{62}$ Parts of this and the following sections have been published in Barrantes (2017)
} 
(§38). Most of my analysis will be focused on the cicada case, since according to most EIA supporters, it is this case that better illustrates their view.

\section{§37. Are MEPPs the best explanations?}

When he first presented his new version of the IA, Baker worried that for the argument to work, there must be genuine mathematical explanations of physical facts, and not only of mathematical facts:

We are interested... in cases where the postulation of mathematical objects yields explanatory power. A key strategic point of the indispensability-based approach is to focus on external applications of mathematics, since otherwise it is open to charges of circularity. Thus we shall not be discussing mathematical explanations of mathematical facts. And since our concern here is with the application of mathematics to science, the explanandum of any putative example must be some physical phenomenon $(2005,225)$.

Now, as we have seen, Melia's objection to the original IA requires that, for the EIA to work, the mathematical explanation should not apply only to a mathematical representation of a physical phenomenon. If the mathematical explanandum was merely a representation of the physical explanandum, then there would be those physical features represented by the mathematics that would actually be doing the real explaining, and the mathematical explanation would be just a proxy to find out about them. So, in order to work as support for mathematical realism, the EIA requires that the MEPP applies to the mathematical features of the physical explanandum. ${ }^{63}$ I will use the cicada case to exemplify this problem and Baker's solution to it. I will then challenge Baker's solution.

\subsection{The concept of primeness in the cicada case}

One important thing to note in the two scientific explanations of the cicada life cycles (see 19.2) is the way the concept of primeness is used. Sometimes primeness refers to a property of time lengths, and sometimes it refers to a property of numbers. The relevant scientific literature does not make this philosophical distinction, and so one important question here is whether we should take scientists at face value and ignore the distinction, or whether we should differentiate between two uses of the word 'prime'.

According to Juha Saatsi, we should not take scientists at face value in this case $(2011,153)$. Whenever the word 'prime' is used in these explanations, it is done with the purpose of picking out the relevant property of time. For Saatsi, the starting point is that "the life-cycle period of North-American cicada [is] exactly 13 or 17 years" (2011, 149). Numbers 13 and 17 are used to represent the fact that "both cicada life-cycles are intersection-minimizing periods" $(2011,153)$. From Saatsi's remarks, I believe the following reconstruction of the cicada case can be advanced ${ }^{64}$.

\footnotetext{
${ }^{63}$ Which, as we will immediately see, implies commitment to the existence of such features.

${ }^{64}$ I am not sure whether this is what Saatsi had in mind, but in any case, this is the interpretation that I defend in this section.
} 
The concept of primeness is responsible for picking out the empirical property of being 'intersection-minimizing periods'. Once we assume that the cycles are evolutionarily advantageous, the explanandum is that the 'life-cycles represented by prime numbers are evolutionarily advantageous', and the explanation must show how this is so.

On the other hand, Alan Baker argues that, in this case, there is no reason to contradict scientists on their use of the term 'prime'. On Baker's view, as we have seen, the life cycles are themselves (mathematically) prime:

Even once biologists had good explanations for the long duration and periodicity of cicada life cycles, they remained puzzled about why these periods have the particular lengths they do. And there is good evidence, based on what they write and say, that this puzzlement only arose because of the fact that both of the known period lengths are prime $(2009,617)$.

According to Baker, then, the explanandum in the cicada case is 'prime life cycles are evolutionarily advantageous'. The explanation consists in showing how the property of primeness provides the desired evolutionary advantage.

This distinction between different descriptions of the explanandum in the cicada case is particularly important for Baker. In the first case, the quantification over mathematical objects can be avoided, but in the second case, the explanandum-claim ineliminably quantifies over mathematical objects. As Baker explains, when one describes the cycles as being 13 and 17 years respectively, one can express the same idea without referring to numbers by using first order logic with identity. For example, a claim such as 'the number of F's is 2 ' can be paraphrased like this:

$$
\exists x \exists y(F x \wedge F y \wedge x \neq y \wedge \forall z(F z \supset(z=x \vee z=y)))
$$

(cf. Baker 2009, 619)

Evidently, an analogous paraphrase can be done for 'the length (in years) of the life cycle of one cicada subspecies is $13^{\prime}$ and 'the length (in years) of the life cycle of the other cicada subspecies is 17.' However, Baker points out, 'the number of F's is prime' cannot be paraphrased away like this. Since there are infinite ways for a number to be prime, the paraphrase would involve an infinite disjunction ('X has life cycle length 2 or length 3 or length 5 or ...') (Baker 2009, 619). Given the fact that scientists do describe the explanandum in terms of primeness, and that there is no nominalist paraphrase of this notion, the particular parts of number theory that have been used in the cicada example are ineliminable, and for this reason, Baker argues, "the mathematics in the [explanation of the] cicada case is indispensable" $(2009,620)$.

Now, given that Baker's goal is to support mathematical Platonism, describing the explanandum in this way is problematic. In an explanation, the explanandum must be true (otherwise, there would be nothing to be explained in the first place). If the explanandum can only be expressed mathematically, one would be already committed to the truth of the mathematical part of it. 
This objection was first pointed out by Mark Steiner. Steiner is one of the first authors who wrote about whether there are genuine MEPPs, and whether their existence would support mathematical realism. He answered affirmatively to the first question, and negatively to the second. According to Steiner, the indispensability argument, even in its explanatory version, begs the question in favor of the mathematical realist:

There are, then, mathematical explanations in physics. Do they make reasonable the existence of mathematical entities? If so, the explananda should not already be committed to such entities. But Goodman and Quine pointed out thirty years ago the apparent impossibility of describing the world without reference to numbers [...] To describe the experience of diversity and change requires mathematical entities [...]

Conclusion: no explanatory argument can establish the existence of mathematical entities (1978b, 19-20).

In an explanation, the explanandum must be true. Steiner's point is that, if the explanandum can only be expressed mathematically, one would be already committed to the truth of the mathematical part of it ${ }^{65}$. Something similar happens in the cicada case. As we have seen, the problem is the role of primeness in the description of the explanandum. For the EIA to overcome Melia's objection, the explanandum itself must be described as being mathematical, which would imply commitment to mathematical realism. But if such is the case, neither the explanation itself would be needed to justify this realism nor would the EIA strategy. Sorin Bangu has recently stressed this point in criticizing Baker's cicada case:

[The explanandum of the cicada case assumes that] there is a mathematical object (specifically: a number) to which the property 'is prime' applies. Therefore, by taking the explanandum as being true... Baker assumes realism before he argues for it $(2008,18)$ (see also Bangu 2012, 157-162).

Bangu's objection shows that if the explanandum in the cicada case is described as Baker does, then we cannot use this case to support mathematical realism. ${ }^{66}$ Baker himself has acknowledged the strength of Bangu's objection:

I suggested... that the concept of primeness is unlikely to be eliminable using only non-mathematical vocabulary. Indeed this is an important part of the reason for thinking that the mathematics in the cicada explanation is indispensable. Combine this with the fact that biologists do tend to phrase the question concerning cicada

\footnotetext{
${ }^{65}$ From the quote above one can see that he believes that that is actually the case. For Steiner, the EIA cannot work precisely because describing the world requires mathematics. My criticism to the EIA does not require believing this. The point is just that if it was the case that the explanandum could only be expressed with mathematics (as Baker believes in the cicada example), then the EIA would be circular.

${ }^{66}$ However, rather than rejecting mathematical realism, Bangu presents the 'banana game' as a case that requires a MEPP, and the explanandum of which is not committed to mathematical entities. I believe the account that I have developed accommodates his example as well, but since it involves probability theory, addressing this example will require a larger discussion than the one I present here.
} 
period length using the concept of primeness, and it seems as if Bangu's complaint is on target $(2009,620)$.

In his (2009), however, Baker presents a reply to Bangu's charge, which I here copy almost in full:

We start with two pieces of data ${ }^{67}$ :

[1] The length (in years) of the life cycle of cicada subspecies $A$ is 13

[2] The length (in years) of the life cycle of cicada subspecies B is 17

These data are acceptable to both the Platonist and the nominalist [...] On the basis of these data, we then advance the following theses:

$\left[1^{*}\right]$ The length (in years) of the life cycle of cicada subspecies A is prime

$\left[2^{*}\right]$ The length (in years) of the life cycle of cicada subspecies B is prime

The mathematical content of $\left[1^{*}\right]$ and $\left[2^{*}\right]$ cannot be paraphrased away [...]. From a philosophical perspective, therefore, we do not at this stage endorse [1*] or [2*] for fear of begging the question [...].

Next we ask whether there is an explanation for the tentative theses, [ $\left.1^{*}\right]$ and $\left[2^{*}\right]$. In discovering the number-theoretic explanation linking primeness to minimization of intersection with other period lengths, we make use of the following intermediate conclusion:

[3] The lengths (in years) of the life cycles of periodical organisms are likely to be prime.

Statement (3) yields a common explanation for [ $\left.1^{*}\right]$ and [2*], from which [1] and [2] follow as specific consequences once appropriate ecological constraints are introduced (Baker 2009, 620-621).

The argument, then, is that the circularity can be avoided if we pay close attention to the way the explanation is actually laid out. According to Baker, the explanandum is indeed that the cycles are, respectively, 13 and 17 (a description that is acceptable to both Platonists and nominalists). But in order to provide a common explanation of the 13 and 17 year cycles, we must tentatively describe the cycles as prime. If this explanation turns out to be better than its alternatives, then the conclusion of the EIA would be supported: the cycles are themselves prime and the explanandum is indeed committed to mathematical objects. This justification, however, would not have been made in a circular way.

\footnotetext{
${ }^{67}$ I have changed the numeration of all the statements, and I am using brackets to indicate this.
} 
Baker then compares this explanation with a hypothetical historico-ecological explanation that would track down all the details of the cicadas' evolutionary history, and concludes that the mathematical explanation is better because it "predicts that other organisms with periodical cycles are also likely to have prime periods" $(2009,621)$, which is the modal aspect that I mentioned in previous sections (specially §29). From the perspective of a historico-ecological explanation, the fact that both cycles are prime would be a coincidence. For Baker, the mathematical explanation is better precisely because it explains why it was somehow necessary for the cycles to end up being prime, and that is how mathematical realism is justified:

Hence, by inference to the best explanation, we ought to believe in the entities invoked in the number theoretic explanation, which includes abstract mathematical objects such as numbers. But once numbers are included in our ontology, we need no longer be tentative about [1*] and [2*] (Baker 2009, 621).

\subsection{The nominalized version of the cicada case}

In Baker's reconstruction of the cicada case, the property that the 13-year cycles and 17-year cycles have in common is the mathematical property of primeness. For Baker, without mentioning this property the explanation would lose explanatory force, because it would be less general and would fail to provide the modal information about the likelihood of periodical species to develop periods that are (described by a number that is) prime. But expressing the property the cycles have in common in terms of primeness is not the only way of providing this generality and modal information. Let us assume that the life cycles are not prime. Rather, they possess a physical property that is responsible for their evolutionary advantage. ${ }^{68}$

As we saw (§21), the mathematical lemmas 1 and 2 in the MEPP were used to pick out the physical relationships expressed by $p$-Lemmas 1 and 2, which can be cashed out in empirical terms using the basic notions of physical combination $(\oplus)$ and equality:

p-Lemma 1: p-coprime iterations minimize overlapping

p-Lemma 2: If $I^{n}(L)$ is p-prime, then it is co-prime with every $I^{m}(L)<I^{2}\left(I^{n}(L)\right)$

The why-question in the cicada case is therefore 'why are the $\mathrm{I}^{13}(\mathrm{~L})$ and $\mathrm{I}^{17}(\mathrm{~L})(\mathrm{L}=$ one year) cycles evolutionarily advantageous?'. The explanation is that they are p-prime, and that, by the two $p$ lemmas above, it follows that the cycles minimize overlapping, which is evolutionarily advantageous. As we saw, p-lemmas 1 and 2 can be explained in terms of physical combination $(\oplus)$ and equality. In the cicada case, time is idealized as linear, and so the time lengths $I^{13}(L)$ and $I^{17}(\mathrm{~L})(\mathrm{L}=$ one year) are $p$-prime with respect to years, and that is why they have the overlapping

\footnotetext{
68 There are many proposed nominalizations of the cicada case in the literature. The nominalization I introduced in section $\$ 19$ does not break new ground in this respect, but it emphasizes how these explanatory facts depend on the extremely simple notions of combination and equality. The simplicity of these two notions will be crucial for defending my point below about these nominalizations providing generality and modal strength.
} 
minimization property, which is the relevant explanatory property of the cycle lengths. It is the instantiation of this property that is responsible for the modal strength of the outcome in the cicada case, and, just as in Baker's version, without mentioning this property it would appear that the life cycles are 13 and 17 by coincidence.

The main idea here is that there is a distinction between the empirical property of $p$ primeness and the mathematical property of primeness. We are trying to explain a scientific claim about insect species, and not an abstract mathematical claim. Do we need to appeal to the (mathematical) property of primeness to express the fact that the cicadas have p-primeness? In one way we do (I explore this below), but even in that case we would be able to paraphrase away such mathematical expression. The problem of the infinite series of disjunctions pointed out by Baker would disappear. Once we are in the domain of application of mathematics, there are constraints on the parts of mathematics that we need to use; so, we are not dealing with pure mathematics any longer. The 'p-primeness' property is a property of time, and the number of ways a life cycle may have this property is not infinite. As opposed to the claim about primeness, there is a finite number of cycles a species may adopt. Expressing this does not involve an infinite disjunction. It is difficult to see, for example, in what way it can be true that 'in principle' a living organism can have a life cycle of, say, 947 years.

The Platonist may protest, however, saying that this is exactly what we mean by 'in principle'. But we can set the number of a cicada cycle (in years) to some integer greater than the known lifetime of the universe (known to be around $13,82 \times 10^{9}$ years) ${ }^{69}$. In that case, we would have only a finite, although very large, disjunctive specification and we can nominalize it. The elements to perform such nominalization already exist.

Now, although it is possible to conceive an empirical version of the cicada case, I believe that this is indeed a MEPP. As we have seen, the distinguishing feature of MEPPs is that they rely on optimal representations; it is for this reason that the mathematics used in them seem to be indispensable: because the explanandum is mathematical. But if it is argued that the MEPP provides the only (or the best) explanation of $\mathrm{P}$ having $\mathrm{P}^{*}$, the reply would be, again, that as long as it is possible to establish a distinction between the mathematical explanandum and the physical explanandum, then the role of mathematics in the explanation would be merely representational. The confusion with MEPPs has been that the distinction between the empirical explanandum and its mathematical representation has not been clearly established in most of the cases discussed. In MEPPs, the mathematical explanation applies to a mathematical representation of $P$, and for that reason these explanations cannot be used to support mathematical Platonism, as proponents of the EIA hold.

The strategy of nominalizing alleged examples of MEPPs on a case by case basis has been called 'piecemeal nominalism' 70 , as opposed to, on the one hand, hard road nominalism and on the other, easy road nominalism. This is, I think, the correct way of describing my view. Although

\footnotetext{
${ }^{69}$ Three years ago the European Space Agency's Planck mission found evidence that the age of the universe might be around that number.

${ }^{70}$ Cf. Baker 2016, 340.
} 
it does not require a full nominalization of all science, it does require, for every alleged case of a MEPP, a clear distinction between the mathematical representation and the empirical set up. In order to do this, we must find a way of nominalizing the explanandum, which is what I did in the cicada case. This is not controversial; in fact, as Bangu has noted, establishing this distinction is also important for the Platonist, otherwise she would be begging the question.

\subsection{Theoretical Virtues}

It could be argued that current scientific practice uses the notion of primeness in the mathematical sense (that is why they use number-theory in the explanation), and that this would be enough to establish the indispensability of mathematics in this case. This objection, however, has already been addressed in the literature; in fact, the answer to this objection is what motivated 'Melia's challenge', which I mentioned above. There are many ordinary scientific explanations that use mathematics; and without using mathematics, they would not be as successful as they are. The same would happen in this case. It would be very hard to pick out the 'p-primeness' property without using mathematics; but from the moment we describe the role of mathematics as merely representational, the alleged indispensability of mathematics would be pragmatic. Mathematics would not be playing an explanatory role - it would be the property represented by the mathematics that would be playing this role. If the presence of mathematics to simplify calculations or to discover new physical properties was all that is needed to justify mathematical Platonism, the detour by the EIA would have been unnecessary. The original indispensability argument would have been enough. That would be a major drawback for defendants of the EIA.

In this section I will focus on other objections, based on whether MEPPs, in the way I have described them, are the best explanations of the phenomena they are about. Davide Rizza has pointed out that, in the cicada case, "the non-numerical explanation... does not suffer of any particular shortcomings with respect to Baker's numerical alternative" $(2011,112)$. I believe this is correct, and in fact I would go further and argue that this is the case for all MEPPs. Baker disagrees. He argues that these nominalized resulting explanations are not the best explanations, because they do not share the theoretical virtues of the mathematical versions. In particular, because they would "lack the generality of the original" $(2016,340)$, and because, by appealing to mathematical objects, Baker's version of this explanation uses facts that are modally stronger than empirical facts (Baker 2016, 16 fn 22).

Below I examine five theoretical virtues (elegance, simplicity, unificatory power, generality, and modal strength) in which, allegedly, the mathematical version performs better than any nominalist version. I will show that this is not the case for any of these five virtues.

\section{a) Elegance}

Let us start with elegance. The idea is that an explanation is better than an alternative if it has greater aesthetical appeal. In the context of this debate, this is perhaps the most problematic notion of the five. First, because intuitions are not clear regarding what it actually amounts to. But also because elegance, understood this way, seems to be reducible to simplicity and unificatory 
power (Colyvan 2001, 79 fn. 25) ${ }^{71}$. Below I will show that my version of the cicada case matches the mathematical version both in terms of simplicity and unificatory power. Therefore, it also matches it in terms of elegance.

\section{b) Simplicity}

One of the most cited theoretical virtues is simplicity: given two theories with the same empirical consequences, we must choose the one that is simpler, that is, that has less ontological commitments (Colyvan 2001, 78.) For Baker, using the notion of mathematical primeness makes for a simpler explanation. He argues $(2016,349)$ that, in the present context, what matters is simplicity with respect to our commitments to concrete posits, regardless of whether this entails overpopulating the world with abstract entities. According to him, even if this explanation would commit us to the whole world of mathematical entities, as long as it has the same empirical commitments as a given alternative, it will not be considered more complex than the said alternative. The question of simplicity, then, comes down to whether the nominalized explanation has the same empirical posits as the mathematical explanation.

In this respect, however, I argue that either postulating mathematical primeness or $\mathrm{p}$ primeness commit us to the same concrete posits. Mathematical primeness would have to be instantiated as a property of concrete time lengths in order to account for the observed cycles; the same happens with the property of p-primeness. Because of this, both explanations are equally simple. Baker has argued that the mathematical explanation is committed to less concrete posits, because it only requires predators of 2 or 3 year cycles $(2016,338)$. But the property of pprimeness would have exactly the same consequence; the only difference being that 2, 3, 13 and 17 would be p-prime; not mathematically prime.

\section{c) Unificatory power}

Another often cited theoretical virtue is unificatory power. Here the idea is to account for the maximum of observed phenomena with the fewer theoretical devices. According to Bangu, for example, quantification over mathematical objects brings the common factor that allows unifying otherwise disparate phenomena $(2012,172)$. In the cicada case, this would mean that by describing the cycles as mathematically prime we would be capable of providing a common explanation of the 13 and 17 cycles. However, this objection does not work against my version of the cicada case. By positing the concrete property of p-primeness we also provide a unified explanation of the two cycles. As we saw, the idea is to unify observed phenomena. In this respect both primeness and p-primeness can do the job - both explanations explain the observed 13 cycles and 17 cycles-, so both explanations are equally unifying.

Now, it may be argued that my piecemeal strategy is less unified than the Platonist strategy, in Philip Kitcher's sense (e.g. 1989). Piecemeal strategies deal with one MEPP at the time, whereas Platonism explicates what is common to all MEPPs. In that sense, the Platonist unifies all MEPPs under the same explanatory pattern: identify the underlying mathematical structure

\footnotetext{
${ }^{71}$ See also Keas (forthcoming), section 5.1.
} 
relevant to explaining the situation, and find out the relevant explanatory property of such structure by a mathematical proof. However, what my account shows is that all alleged cases of MEPPs can be understood in the same way: first, we must establish a clear distinction between the physical explanandum and its mathematical representation, and next we must show that the mathematical derivation is actually tracking down the relevant physical explanatory features. So, although it is true that I propose that we have to examine MEPPs one by one, the explanatory pattern used to make sense of these explanations is the same. So even at this level my proposal is as unified as the Platonist one.

\section{d) Generality}

As we have seen, Baker argues that the mathematical version is more general than the nominalist version. The generality objection runs as follows: postulating mathematical primeness accounts for a wider range of possible observations, and because of that, even if we nominalize the explanandum in the way proposed by piecemeal nominalists, the explanation that appeals to mathematical primeness is the best one (e.g. Baker 2009, 617; 2016, 340). But how much generality do we really need? As we saw, there are empirical constrains that limit the possible length of the cycles. For starters, they cannot be longer than the age of the universe. What is more, the overall explanation of the cicadas' life cycles depends on the fact that during the Pleistocene the Earth temperature was extremely cold, and that that is why cicadas developed relatively long cycles. Therefore, it is a condition for the evolutionary explanation to work that the cycles are less than 11700 years.

It may still be objected that the interesting thing about the explanation that appeals to primeness is that it would work in other planets older than ours, and that it is precisely this generality that would get lost if we do not use the notion of mathematical primeness. But, again, those planets would still be physical, just as ours. And however old these planets may be, their age would still be measurable in terms of finite cycles. Moreover, however long the cycles are, they cannot be infinitely long if we are talking of live species, because the very idea of an infinite life cycle does not make sense in this context - if a time length does not have an upper bound, it would not make sense to call it 'a cycle'.

Now, the Platonist may still reply that the result is still less general than when we describe the explanandum as prime, even if we do not appeal to infinitely long cycles, because it would apply to more cycles than mere physical or realizable cycles. But I do not think this is going to be enough to justify the existence of those non-realizable cycles. Consider an example due to James Franklin $(2014,69)$. The statement 'all red things are colored' applies to all red things, and we can say that for anything, if it was red, then it would be colored. However, it does not seem right to justify the existence of infinitely many red things just because if there were infinitely many red things the statement 'all red things are colored' would be more general. In the same vein, it does not seem right to justify the existence of numbers just because if there were numbers, the statement ' $n$ is prime' would be more general. If this were enough to justify the existence of numbers, the detour around the EIA would have been unnecessary (I return to this point below). 
Also, it is important to note that what mine and similar nominalizations of the cicada case show is that the explanation relies on the simple notions of combination and equality, which are general enough properties. They apply to any set of objects once they are described in a certain way. This generality can be captured in modal terms. For example, if there are two objects of congruent lengths then the notion of equality would apply to them. But again, this by itself does not justify the existence of infinite objects that fit this description.

\section{e) Modal strength}

Finally, it may be argued that the kind of modal information provided by mathematical properties is stronger than any provided by empirical properties (Baker 2016, 16 fn. 22; Lyon 2011, 9-10). Thus, the fact that primeness minimizes intersection between numbers is mathematically necessary. This necessity would be stronger than the fact that p-primeness, a property of time lengths, minimizes intersection between biological species. Therefore, appealing to these 'stronger necessities' would provide a better explanation than appealing to the weaker physical necessities. This interpretation presupposes the existence of mathematical necessities in the physical world. In that sense, the explanation that appeals to mathematical primeness would highlight the fact that it is mathematically necessary that prime-numbered cycles minimize intersection with other cycles. This would be true in any world where the idea of cycles makes sense, so this necessity would be stronger than physical necessity (this is the view endorsed by Lange (2013); however, he does not link it to the indispensability debate).

The problem with this suggestion is that whether or not this justifies mathematical Platonism is independent of the Indispensability Argument. Let me illustrate this with Franklin's example. 'All red things are colored' is necessarily true, it applies to things in the real world, and it would still be true in any world where red is a color. So even though this is a fact about the physical world, it is necessarily true in a way stronger than physical necessity: even if the laws of nature were different, it would be true that 'all red things are colored' (Franklin 2014, 69). But again, the fact that 'all red things are colored' is necessarily true does not by itself prove that the property 'red' exists independently of its realizations. And the fact that 'in every world where red is a color, red things would be colored' does not by itself prove that those other worlds actually exist. In the same way, maybe some relations between time lengths are modally stronger than other physical relations $^{72}$; but whether or not the existence of relations of necessity stronger than physical necessity shows that there exist abstract mathematical objects is a deep metaphysical question, which answer seems to be independent of considerations about the usefulness of mathematics in science, which is what the EIA is ultimately about.

Let us see how this works in the cicada case. I have shown that the empirical explanation of the life cycles' length relies on the extremely simple notions of combination and equality of physical lengths. If we describe physical objects in a way such that combination and equality apply, then every physical object that fits such definition would be subject to these relations of

\footnotetext{
72 Perhaps geometrical relations are physical and yet stronger than mere nomologically necessary. I cannot go into more details about this at this point. However, I hope my overall strategy is clear: the existence of geometrical relations does not by itself support mathematical Platonism.
} 
combination and equality, which in turn entails that any physical object represented by 13 would not overlap with iterations of other lengths represented by 26 or less, apart from those represented by 1 ; similarly, it entails that any physical object represented by 17 would not overlap with iterations of other lengths represented by 34 or less, apart from those represented by 1 . This will be necessarily true. But the crucial point is this: even if this relationship turns out to be that of mathematical necessity, it would be as surprising as the relationship of necessity between, say, the fact that if there were two objects of the same length, and a third object of the double length of the first object, then a combination of the lengths of the first two would be equal to the length of the third. If this fact were enough to support mathematical Platonism, then the detour by the EIA would have been unnecessary. Again, I do not want to say that these are not mathematical relationships of necessity in the world ${ }^{73}$; only that whether or not they are is independent of naturalism and the usefulness of mathematics in science, which is what the EIA is about. In other words, the usefulness of mathematics in science has nothing to do with this metaphysical claim.

This point is perhaps clearer in the bridges of Königsberg case. The impossibility of performing a trip that crosses all the seven bridges of $18^{\text {th }}$-century Königsberg without retracing one's steps is explained by a graph-theoretical theorem. For me, what is crucial for understanding this case is that Euler's proof of this theorem relied on the extremely simple fact that every time one crosses a bridge, two pieces of land are involved, the starting point and the ending point. As we have seen, it is often said that it is a matter of mathematical necessity that the trip over the bridges is impossible. This necessity would be both stronger than mere physical necessity, and about a physical system (cf. Lange 2013; Lyon 2011). As I said, I prefer not to enter this debate here. My point is that if the impossibility of performing an Eulerian trip over the bridges is described as a matter of mathematical necessity, then the fact that 'every time that I cross a bridge two pieces of land are involved' would also be mathematically necessary. But if this justifies mathematical Platonism, such an argument does not need the complications of the EIA, or for that matter, of issues pertaining the applicability of mathematics in science.

The defining feature of MEPPs is that they rely on optimal representations. These are cases where researchers are interested in special features of a physical situation, such that all that is needed to explain these features can be included in the mathematical representation. In these explanations, the role of mathematics, although perhaps pragmatically indispensable - in the sense that it would have been very unlikely that researchers would have discovered the relevant explanatory factors without using mathematics -, is ultimately representational. For that reason, these cases do not overcome Melia's challenge, and so they do not support the conclusion of the EIA.

\section{$\S 38$. The EIA and practical indispensability}

We have seen that in MEPPs explanatory derivations are indispensable because MEPPs rely on optimal representations, and so the explanatory strength comes purely from the mathematical derivation of the mathematical facts in the representation. This is a sense in which mathematics

\footnotetext{
${ }^{73}$ I believe there are not, but I do not think this is relevant for my overall case against the EIA supporting Platonism.
} 
is indispensable: explanatory derivations are indispensable for MEPPs to work as explanations. Given my characterization of MEPPs, this sense of indispensability is not the one required by the EIA because in my account it is possible to conceive an ordinary physical explanation of the same facts. But there is another sense in which MEPPs can be indispensable, at least in the cases that I have discussed. So, let us focus on this issue:

There are two ways in which mathematics contributes to the explanations of the cicada case and the bridges case. The first is by making these problems computationally tractable, and the second is by facilitating the discovery of modally stronger facts. Some of these contributions, depending on the case, can be indispensable to finding an explanation of the physical explananda (I will call it 'practical indispensability'). In this section, I explore this other sense of indispensability and show that it is not the one required by the EIA either. Ultimately, as I will show, this pragmatic indispensability does not entail that mathematics itself is explanatory. My view is compatible with Saatsi's, who has pointed out that:

[T]he cases discussed in the literature demonstrate that mathematics can play a knowledge-conferring role in science: it can help us learn about the world. But the fact that mathematics can give us knowledge (or better justified beliefs) of certain physical facts does not automatically entail that it thereby plays an explanatory role (Saatsi 2011, 1445).

We saw that one of the cases where the IBE principle does not apply is if a posit does not play the right role in the explanation. This is exactly the case of MEPPs. Mathematics, even when it is indispensable in practice, always plays these knowledge-conferring role, in virtue of representing and helping draw inferences about the physical explanatory facts. The contributions of mathematics to the two cases I discussed in previous chapters fall precisely under this 'knowledge-conferring' role.

The first contribution is computational tractability. This can be seen in the bridges case if we try to solve the original problem following a 'brute computation strategy'. As we saw, this strategy consists in systematically performing every single possible route over the bridges. In doing so, we would notice that none of the routes succeeds in crossing the seven bridges using each bridge only once and so we would be able to conclude that such a route is impossible. However, as we saw, Euler himself pointed out that this strategy works for the bridges of Königsberg, but it will not work for other, more complicated cases. One such case is that of the bridges of Venice, where the brute computational strategy is unfeasible because there are 420 bridges, which gives a total of possible routes that cannot be computed. As we saw (§18), the use of mathematics makes this and similar cases accessible. This is one sense in which mathematics may be considered indispensable for solving those more complicated cases: mathematics makes those cases computationally tractable. This contribution is compatible with nominalism because it is similar to counting.

The second contribution of mathematics is showing the modal strength of the explanandum. This is exemplified by both the bridges case and the cicada case. As we saw (§18), although in the bridges case the brute computational strategy may verify that all possible routes 
are unsuccessful, this method fails to identify the common feature of these routes that renders them unsuitable as Eulerian paths. Thus, from the perspective of the brute computational strategy, the fact that all possible attempts failed in the Königsberg case is a mere coincidence. The mathematics, on the other hand, shows that the result is modally stronger than could have been rendered by any particular unsuccessful attempt: the bridges system does not have the required structural features (conditions $\mathrm{C} 1$ and $\mathrm{C} 2$ ) for such a path to be possible, leading to a lack of a successful route. It is clear that the role of mathematics here is to represent the physical structures relevant to the possibility, or lack thereof, of performing an Eulerian path, and to show how these structures do not allow such a path.

It is also possible to conceive of an alternative explanation of the cicada's life cycle. For example, one may eventually identify the specific events that affected the cicada's evolutionary history; or one could even identify the biological basis of the cicada's behavior. Nevertheless, as we have seen (§19), this alternative explanation would make it look as if the cycles were somehow accidental. By using mathematics we can show that, independently of the evolutionary history of the cicadas, these insects were likely to evolve prime life-cycles because these cycles have the property of minimizing the possibility of intersection with other species. It is only by appealing to this property that we can explain, as Baker puts it, "why any periodical organism with periodical predators is likely to evolve a life-cycle period that is prime" (2012, 257; my emphasis). Thus, another sense in which mathematics may play an indispensable role is that the mathematical explanation reveals the modal strength of the explanandum ${ }^{74}$.

But in principle, one could have discovered these modally stronger facts of the physical systems in question without using mathematics. For example, it is not impossible to conceive of a person discovering the relevant structural facts about the bridges system by attentively observing the bridges system from the top of the Königsberg Cathedral and discovering the relations between this and the modally stronger fact that 'a bridge connects two pieces of land'. In the same way, we can conceive of some alternative means one may have discovered the relevant facts about time that operate in the cicada case. As Saatsi has pointed out, the idea is that there is an intersection-minimizing period $T_{x}$ for periods in the range $\left[T_{1} \ldots T_{2}\right]$. One could have identified such a period $T_{x}$ by manipulating sticks with proportional measurements (cf. Saatsi 2011, 152), and thus discovering the relation between this and the modally stronger notions of combination and equality.

However, even if such alternative means of discovery are conceivable, it is not plausible that one may actually come to know the facts about road systems or life-cycles without mathematics. It seems very unlikely that scientists would have identified these modal facts about the world without the assistance of mathematics. For example, by correctly representing the relevant relations in the system of bridges, one can infer why it is impossible to perform an Eulerian path. This is even more evident in the cicada case. We have seen that it seems hard enough even to express those facts without mathematics. The use of the natural number series as a model for the sequence of years seems to be indispensable for discovering the facts about time that explain

\footnotetext{
${ }^{74}$ As we have seen (chapter 4), Lange 2013 and Lyon 2011 also emphasize this point.
} 
the length of animal life-cycles. But once we envision a way to discover these modal facts that does not presume the use of mathematics, no matter how implausible this way is, the use of mathematics becomes merely pragmatic. By correctly representing the relevant features of time, mathematics helps us infer other facts about time that explain this case.

As we have seen, in these explanations mathematics seems to play an indispensable role. Is this the kind of indispensability required by the first premise of the EIA? I argue that it is not. My discussion shows that mathematics is indispensable to find out truths about the relevant explanatory physical facts. Computational tractability is clearly a pragmatic notion. And even though modal strength is not, we have seen that modal strength can be accounted for in terms of the necessities associated with the relevant physical systems. The indispensable role of mathematics in the process of discovering these necessities is also a pragmatic contribution. Therefore, although mathematics may play an indispensable role in these explanations - because it offers good representations of the relevant explanatory features of the physical systems involved - this practical indispensability is not ontologically committing in the sense required by the EIA; it ultimately depends on the representational and inferential roles of mathematics. In other words, premise P2* of the EIA is about practical indispensability, but this is not the sense of indispensability required by premise $\mathrm{P} 1^{*}$, which is the one that, allegedly, carries ontological weight. In the cases I have studied, mathematics has proven to be an indispensable knowledgeconferring tool, and as we saw (§36), both sides of the debate agree that this role of mathematics does not commit us to beliefs in the existence of mathematical objects.

\section{$\S 39$. Conclusion}

It is important to note, for the purpose of assessing the EIA in light of MEPPs, that in these explanations mathematics is representing features of the physical systems in question, and it helps us to infer facts about these features. Even if it may have been practically impossible to do this without mathematics, this is no reason to believe that the role of mathematics is in itself explanatory. As Saatsi points out:

[M]athematics only plays a role in representing physical facts... allowing us to infer certain physical facts from other physical facts, and hence providing us knowledge of the crucial explanatory physical fact (Saatsi 2011, 146).

It is true that MEPPs are scientific explanations that rely on explanatory derivations, but this is because the physical setup can be optimally represented with respect to the given explanandum for each case. As I have shown, the role of mathematics in these optimal representations is not ontologically committing. In this respect, there is no difference between the role of mathematics in ordinary explanations that rely on either Galilean or minimal model idealizations, and those that rely on optimal representations, since both improvable and nonimprovable representations capture or highlight the relevant (physical) explanatory facts.

As I mentioned at the beginning of this chapter, my discussion of the IA and the role of MEPPs in the EIA debate is not a positive argument for mathematical antirealism. Baker is correct when he says that: 
[A]n argument... that purports to show how appeal to mathematical objects could be explanatory with respect to some given physical phenomena despite the mathematical objects not existing is not-or at least not obviously-an argument for suspending belief in the existence of such objects (Baker 2009, p. 627).

I fully agree with Baker here. But showing that we can explicate MEPPs without appealing to mathematical objects constitutes an argument against the Enhanced Indispensability Argument. If the role of mathematics in MEPPs is ultimately representational, then the presence of MEPPs in science is not a good reason to believe in the existence of mathematical entities. 


\section{REFERENCES}

Baker, A. (2005), 'Are there Genuine Mathematical Explanations of Physical Phenomena?', Mind, 114: 223-238.

(2009a), 'Mathematical Explanation in Science', British Journal for the Philosophy of Science, 60: 611-633.

(2009b), 'Only connections?', Penn Humanities Forum 2009-10. Retrieved from: www.swarthmore.edu/.../PHF\%20Baker\%20only\%20connections.pdf

--------- (2012), 'Science-Driven Mathematical Explanation', Mind 121 (482): 243-267.

(2016), 'Parsimony and Inference to the Best Mathematical Explanation', Synthese: 1-18.

Bangu, S. (2008), 'Inference to the Best Explanation and Mathematical Realism', Synthese, 160, 13-20.

(2012), The applicability of mathematics in science: indispensability and ontology, New York: Palgrave Macmillan.

Bangu, S. (2013), 'Indispensability and Explanations', British Journal for the Philosophy of Science, 64 (2): 255-277.

Barnett, J., (2005), Early Writings on Graph Theory: Euler Circuits and The K"onigsberg Bridge Problem An Historical Project. (Retrieved from: http://www.math.umn.edu/ reiner/Classes/Konigsberg.pdf)

Barrantes, M. (2017), 'Optimal Representations and the Enhanced Indispensability Argument', Synthese. (https://doi.org/10.1007/s11229-017-1470-4)

Batterman, R., 'On the Explanatory Role of Mathematics in Empirical Science', Br J Philos Sci 2010; 61 (1): 1-25.

Beebee, H. et al. (Ed.), The Oxford Handbook of Causation, Oxford: Oxford University Press.

Benacerraf, P. and H. Putnam (eds.) (1983), Philosophy of mathematics. Selected Readings 2nd Edition, Cambridge: Cambridge University Press.

Biggs et al., (1998), Graph Theory 1736-1936, Oxford, Clarendon Press.

Bogen, J. and Woodward, J. (1988), 'Saving the Phenomena', Philosophical Review, 97: 303- 352.

Brown, J.R. (2008), Philosophy of Mathematics: A Contemporary Introduction to the World of Proofs and Pictures, New York: Routledge. 
Bueno, O. (2005), 'Dirac and the dispensability of mathematics', Studies in History and Philosophy of Modern Physics 36. 465-490.

(2009), 'Mathematical Fictionalism', in Bueno and Linnebo, Eds. (2009)

(2012). 'An Easy Road to Nominalism', Mind, Vol. 121.484.

(2014), "Nominalism in the Philosophy of Mathematics", The Stanford Encyclopedia of Philosophy (Spring 2014 Edition), Edward N. Zalta (ed.), URL = <https://plato.stanford.edu/archives/spr2014/entries/nominalism-mathematics/>.

Bueno, O. and M. Colyvan (2011), 'An Inferential Conception of the Application of Mathematics', Noûs, 45 (2): 345-374.

Bueno, O. and S. French (2011), 'How Theories Represent', British Journal for the Philosophy of Science, 62 (4): 857-894.

-------- (2012), 'Can Mathematics Explain Physical Phenomena?', British Journal for the Philosophy of Science, 63 (1): 85-113.

-------- (2017), Applying Mathematics: Immersion, Inference, Interpretation, Oxford: Oxford University Press.

Bueno, O. and Linnebo, $\varnothing$. Eds. (2009), New Waves in Philosophy of Mathematics, New York: Palgrave Macmillan.

Busch, J. (2012), 'Can the new indispensability argument be saved from Euclidean rescues?', Synthese, 187:489-508.

Cargile, J. (2003), 'On Alexander's Dictum', Topoi 22: 143-149.

Colyvan, M. (2001), The indispensability of mathematics, Oxford: Oxford University Press.

--------- (2007), 'Mathematical Recreation Versus Mathematical Knowledge', in Leng et al (2007) (2010), 'There is no Easy Road to Nominalism', Mind 119 (474): 285-306.

--------- (2012), An Introduction to the Philosophy of Mathematics, Cambridge: Cambridge University Press.

Cooley, John, Magicicada.org (consulted on Sept 2016)

Cox, T., and C. Carlton (2003), 'A Comment on Gene Introgression versus En Masse Cycle Switching in the Evolution of 13-Year and 17-Year Life Cycles in Periodical Cicadas', Evolution, Vol. 57, No. 2, pp. 428-432. 
Craver, C. and M. Povich (2017), 'The directionality of distinctively mathematical explanations', Studies in History and Philosophy of Science Part A, 63: 31-38.

da Costa, N. and S. French (2003), Science and Partial Truth: A Unitary Approach to Models and Scientific Reasoning, Oxford: Oxford University Press

Daly, C. and S. Langford, 'Mathematical explanation and indispensability arguments', Philosophical Quarterly 59 (237):641-658.

Demopoulos, W. and M. Friedman (1985), 'Bertrand Russell's The Analysis of Matter: Its Historical Context and Contemporary Interest', Philosophy of Science, Vol. 52, No. 4 621-639

Dowe, P. (2009), 'Causal Process Theories', in Beebee, H. et al. (Ed.) (2009).

Euler, L. 'Solutio Problematis Ad Geometriam Situs Pertinentis', in Barnett (2005)

Field, H. (1980), Science without Numbers: A defense of Nominalism, Princeton: Princeton University Press. (1989), Realism, Mathematics \& Modality, Oxford: Basil Blackwell.

Franklin, J., An Aristotelian Realist Philosophy of Mathematics. Mathematics as the Science of Quantity and Structure, Palgrave Macmillan UK.

Goles, Schulz and Markus (2001), 'Prime number selection of cycles in a predator-prey model', Complexity, 6, $33-38$.

Hall, Ned (2004), "Two Concepts of Causation," in: Hall, N., Collins J.and Paul, L.A. (2004), pp. 225276.

Hall, N., Collins J.and Paul, L.A. (eds.), Causation and Counterfactuals, MIT Press, Cambridge.

Hempel, C. (1965), Aspects of Scientific Explanation and other essays in the philosophy of science, New York, Free Press.

-- (1983), 'On the nature of mathematical truth', in Benacerraf, P. and H. Putnam (eds.) (1983)

Hempel, C. and P. Oppenheim (1948), 'Studies in the logic of explanation', Philosophy of Science, 15 (2): 135-175.

Hitchcock, C. (1995), 'Salmon on Explanatory Relevance', Philosophy of Science 62 (2):304-320.

Hopkins, B and R. Wilson (2004), 'The Truth About Konigsberg', The College of Mathematics Journal, V. 35, N. 3. 
Humphreys, P. (1989a), 'Scientific Explanation: The causes, some of the causes, and nothing but the causes', Minnesota Studies in the Philosophy of Science. Vol XIII. Scientific Explanation, 13:283-306.

--------- (1989b), The Chances of Explanation. Causal Explanation in the Social, Medical, and Physical Sciences, Princeton, Princeton University Press.

-- (1995), 'Abstract and Concrete', Philosophy and Phenomenological Research, 55 (1):157161.

------ (2004), Extending Ourselves, Oxford: Oxford University Press.

--------- (2006) 'Review Symposium. Invariance, Explanation and Understanding,' Metascience 15, pp. 39-44.

Jackson, F. and P. Pettit (1990), 'Program Explanation: A General Perspective', Analysis 50/2: 10717.

Keas, M. (Forthcoming), 'Systematizing the theoretical virtues', Synthese.

Kennedy, A. (2012), Models and Idealization in Scientific Explanation, Unpublished PhD Dissertation, University of Virginia.

Kitcher, P. (1981), 'Explanatory Unification', Philosophy of Science, 48 (4): 507-531

Kitcher, P. (1989), 'Explanatory Unification and the Causal Structure of the World', Minnesota Studies in the Philosophy of Science. Vol XIII. Scientific Explanation, 13:283-306.

Lange, M. (2010), "Why Proofs by Mathematical Induction are Generally not Explanatory", Analysis, 69: 203-211.

--------- (2013), 'What Makes a Scientific Explanation Distinctively Mathematical?', British Journal for the Philosophy of Science, (64 (3):485-511.

------ (2014), 'Aspects of Mathematical Explanation: Symmetry, Unity, and Salience, Philosophical Review, 123(4): 485-531

Liggins, D., (2012), 'Weaseling and the content of science', Mind, 121 (484): 997-1005.

Lyon, A. (2011), 'Mathematical Explanations Of Empirical Facts, And Mathematical Realism, Australasian Journal of Philosophy 90 (3):559 - 578.

Maddy, P., 'Three forms of Naturalism', in Shapiro (2005)

Mancosu, P. (2001), 'Mathematical Explanation: Problems and Prospects', Topoi, 20: 97-117. 
------ (2008), 'Mathematical Explanation: Why it Matters'. In Mancosu, P. (ed.), The Philosophy of Mathematical Practice, Oxford: Oxford University Press.

- (2011), 'Explanation in Mathematics', The Stanford Encyclopedia of Philosophy (Summer 2011 Edition), Edward N. Zalta (ed.), URL = $<$ http://plato.stanford.edu/archives/sum2011/entries/mathematics-explanation/>.

Matson, J. (2013), 'Deciphering the Strange Mathematics of Cicadas', Scientific American, May 23, 2013.

McMullin (1985), 'Galilean Idealization', Studies in History and Philosophy of Science, XVI, 247-273.

Melia, J., (2000), "Weaseling Away the Indispensability Argument", Mind, 109(435): 455-479 -- (2002): 'Response to Colyvan'. Mind, 111, pp. 75-79.

Nagel, E. et al (Eds.) (1962), Logic, Methodology, and Philosophy of Science: Proceedings of the 1960 International Congress, Stanford: Stanford University Press.

Newman, M.H.A. (1928), 'Mr. Russell's Causal Theory of Perception”, Mind 37: 137-48.

Orzack, S. and E. Sober (2001), Adaptationism and Optimality, Cambridge: Cambridge University Press.

Pincock, C. (2005), 'Overextending Partial Structures: Idealization and Abstraction', Philosophy of Science 72, no. 5: 1248-1259.

--------- (2007), 'A Role for Mathematics in the Physical Sciences', Noûs, 41, pp. 253-75.

-------- (2011a), 'Modeling Reality', Synthese, 180 (1):19 - 32

-(2011b), 'On Batterman's 'On the Explanatory Role of Mathematics in Science', British Journal for the Philosophy of Science, 62: 211-217.

--------- (2012), Mathematics and Scientific Representation, Oxford: Oxford University Press.

- (2015), 'Abstract explanations in science', British Journal for the Philosophy of Science 66 (4): 857-882.

Quine, W.V. (1991), 'Two Dogmas in Retrospect, Canadian Journal of Philosophy, 21, 3: 265-274.

Resnik (1997), Mathematics as a Science of Patterns, Oxford: Oxford University Press.

Resnik, M. (2005), 'Quine and the Web of Belief', in Shapiro (2005)

Resnik, M. and D. Kushner (1987), 'Explanation, independence and realism in mathematics', British Journal for the Philosophy of Science 38 (2):141-158. 
Rice, C. 'Moving Beyond Causes: Optimality Models and Scientific Explanation', Noûs , 49 (3): 589 615.

Rizza, D. (2011), 'Magicicada, Mathematical Explanation and Mathematical Realism', Erkenntnis, 74 (1):101-114

Saatsi, J. (2011), 'The Enhanced Indispensability Argument: Representational versus Explanatory Role of Mathematics in Science', British Journal for the Philosophy of Science, 62 (1): 143154.

--------- (2012), 'Mathematics and Program Explanations', Australasian Journal of Philosophy, 90 (3):579-584.

-------- (2016), 'On the 'Indispensable Explanatory Role' of Mathematics', Mind, 125 (500):10451070.

Saatsi, J. and M. Pexton (2013), 'Reassessing Woodward's Account of Explanation: Regularities, Counterfactuals, and Noncausal Explanations', Philosophy of Science, 80 (5): 613-624.

Salmon, W. (1984), Scientific Explanation and the Causal Structure of the World, Princeton, Princeton University Press.

(1998), Causality and Explanation, Oxford, Oxford University Press.

-------- (2000), 'The Spirit of Logical Empiricism: Carl G. Hempel's Role in Twentieth-Century Philosophy of Science', in Fetzer, J. (Ed.), Science, Explanation, and Rationality. The philosophy of Carl G. Hempel, Oxford: Oxford University Press.

-------- (2006), Four Decades of Scientific Explanation, Pittsburgh, University of Pittsburgh Press.

Shapiro, S. (1997), Philosophy of mathematics. Structure and ontology, New York: Oxford University Press.

Shapiro, S. (Ed) (2005), The Oxford Handbook of Philosophy of Mathematics and Logic, Oxford: Oxford University Press.

Steiner, M., 1978a, "Mathematical Explanation", Philosophical Studies, 34: 135-151.

1978b, "Mathematics, Explanation, and Scientific Knowledge", Noûs, 12: 17-28.

Suárez, M. (2010), 'Scientific Representation', Philosophy Compass 5/1: 91-101.

Suppes, P. (1962), 'Models of Data', in Nagel, E. et al (Eds.) (1962).

Tan, P., 'The Challenge of Inconsistent Idealizations' (unpublished draft)

van Fraassen, B. (1980), The Scientific Image, New York: Oxford University Press. 
Weisberg, M. (2007a), 'Three Kinds of Idealizations', Journal of Philosophy, 104 (12):639-659

-------- (2007b), 'Who is a Modeler?', British Journal for the Philosophy of Science, 58 (2):207-233. (2013), Simulation and Similarity. Using models to understand the world, Oxford, Oxford University Press

Woodward, J. (2003), Making Things Happen. A theory of Causal Explanation, Oxford, Oxford University Press.

-------- (2006) 'Review Symposium. Invariance, Explanation and Understanding,' Metascience 15, pp. 53-66.

-------- (2009), 'Agency and Interventionist Theories', in Beebee, H. et al. (Ed.) (2009).

(2013), "Causation and Manipulability", The Stanford Encyclopedia of Philosophy (Winter 2013 Edition), Edward N. Zalta (ed.), URL = <http://plato.stanford.edu/archives/win2013/entries/causation-mani/>. 